\title{
RESISTIVE INDEX IN OBSTRUCTIVE UROPATHY
}

\author{
Resistentie-index in obstructieve uropathie
}

\section{PROEFSCHRIFT}

ter verkrijging van de graad van doctor aan de Erasmus Universiteit Rotterdam op gezag van de rector magnificus

Prof. dr. P.W.C. Akkermans M.A. en volgens het besluit van het College voor Promoties.

De openbare verdediging zal plaats vinden op woensdag 3 maart 1999 om 15.45 uur.

door

\section{Ahmed Abdurrahman Shokeir}

geboren te El-Badrasheen, Geza, Egypte 


\section{PROMOTIECOMMISSIE}

Promotor: $\quad$ Prof. dr. F.H. Schröder

Overige leden: $\quad$ Prof. dr. H. Buller

Prof. dr. A.J. van der Heijden

Prof. dr. L.A.H. Monnens

Co-promotores: $\quad$ Dr. J.M. Nijman

Dr. A.P. Provoost

This PhD-thesis has been supported by a grant from the S.U.W.O. (scientific research foundation department of Urology Erasmus University Rotterdam). 


\section{CONTENTS}

Page

Chapter 1

Introduction and scope of the thesis

1.1 Resistive Index in obstructive uropathy

1.2 Recoverability of renal function after relief of upper

chronic partial urinary tract obstruction.

1.3 Scope of the thesis

Chapter 2

Partial ureteral obstruction: a new variable and reversible canine experimental model

Chapter 3

Partial ureteral obstruction: effect of intravenous normal saline and furosemide upon the renal resistive index.

Chapter 4

Partial ureteric obstruction: a study of Doppler ultrasonography and diuretic renography in different grades and durations of obstruction.

Chapter 5

Partial ureteral obstruction: role of renal resistive index in stages of obstruction and release

\section{Chapter 6}

Renal Doppler ultrasound in children with normal upper urinary tracts: effect of fasting, hydration with normal saline, and furosemide administration. 


\section{CONTENTS}

Chapter 7

Renal doppler ultrasound in children with obstructive uropathy: effect of intravenous normal saline fluid load and furosemide

Chapter 8

Renal doppler ultrasound in children with equivocal obstructive uropathy: effect of intravenous normal saline fluid and furosemide.

Chapter 9

General discussion

References

Summary

Samenvatting

Curriculum vitae

List of publications

136

Dankwoord/acknowledgements 
Introduction and Scope of the Thesis

1.1 Resistive index in obstructive uropathy

Ahmed A. Shokeir, Abraham P. Provoost and Rien J.M. Nijman

Urology \& Nephrology Center, Mansoura University, Mansoura, Egypt and Departments of Pediatric Surgery And Pediatric Urology, University Hospital, Erasmus University, Rotterdam, The Netherlands

Br. J. Urol. (1997) 80: 195-200 

The diagnosis of urinary tract obstruction is a difficult and perplexing problem particularly in children. Pyelocalyectasis is seen not only in obstruction but also in other conditions, such as residual dilatation after relief of obstruction, vesicoureteral reflux and pyelonephritis. Grey-scale ultrasonography is of little value in this clinically important distinction. The standard excretory urography (IVU), even with diuretic augmentation, does not permit the objective diagnosis or exclusion of urinary tract obstruction. The Whitaker test is considered by some authors to be the gold standard for the diagnosis of obstructive pyelocalyectasis but it is invasive and therefore has not gained wide use. Moreover, the intrinsic urine output of the kidney contributes an unknown volume to the total amount of fluid being infused, particularly in children, and the potential for false-positive results should be considered whenever the urine output of the corresponding kidney is high. Finally, the results are not always reproducible or consistent with surgical findings.

Nowadays, diuretic renography is the most widely accepted non-invasive procedure to diagnose obstruction. However, it has the disadvantages of being expensive, using ionising radiation and having a $10 \%-15 \%$ rate of false-positive and indeterminate results (Kass et al., 1985). Magnetic resonance imaging (MRI) (Thurnher et al., 1989) and various biochemical indicators of the response of the kidney to obstructive damage (Carr et al., 1994) have recently been investigated. However, the clinical significance of such new approaches remains to be determined.

The potential usefulness of the resistive index (RI) obtained during Doppler ultrasonography (DU) was recently described by Platt et al (1989a). The non-invasive nature of measurement of renal RI gives it considerable appeal in its potential application to patients with obstructive uropathy. The present paper will review the current trends of the role of RI in the diagnosis of obstructive uropathy.

\section{RI: what does it mean?}

In 1979, Arima et al. used DU to identify an alteration in arterial blood flow in renal allografts undergoing rejection. This finding was accompanied by an increase in renal arterial resistance resulting from a decrease in diastolic blood flow greater than the systolic flow. The RI is thus defined as: (peak systolic velocity - lowest diastolic velocity )/ peak systolic velocity. 
Rifkin et al. (1987) identified acute rejection based on the measurement of renal RI. The use of RI in the diagnosis of obstructive uropathy is based upon the physiologic characteristic that a decrease in renal blood flow and an increase in renovascular resistance are the hallmarks of significant obstructive uropathy (Vaughan et al., 1970). Platt et al. (1989a) recently observed that ureteric obstruction with hydronephrosis produced changes in Doppler waveforms, whereby an increase in downstream resistance resulted in a more marked reduction in diastolic blood flow compared to the systolic component. This difference causes an increase in RI.

\section{Technique of Doppler ultrasonography}

The application of an appropriate technique is essential for obtaining accurate results. Patients should be well hydrated and indwelling urethral catheter should be placed to ensure absence of bladder pressure artifacts. Most examinations are performed with 2.25, 3, 3.5 or $3.75 \mathrm{MHz}$ tranducers. The Doppler sample volume is set to $2 \mathrm{~mL}$ at $5 \mathrm{~mm}$ and placed at corticomedullary junction of the kidney (arculate asteries) or along the border of medullary pyramids (interlobar arteries). These extremely small vessels have relatively low velocities with associated small frequency shifts and thus if the wrong Doppler settings are used, only slight deflections from the baseline will be produced, making accurate resistance measurements more difficult. To offset this potential problem, the lowest wall filter for the particular machine should be used. Perhaps more importantly the Doppler examination should be carried out using a scale with the smallest possible frequency range (minimum pulse repetition frequency) that does not produce aliasing (Platt et al., 1991). This gives the highest sensitivity to low flow and generates a spectrum that fills as much of the scale as possible. Measurements made from Doppler spectrum that barely deviate from the baseline will be highly variable and can produce critical measurement errors (Platt et al., 1991).

To characterize intrarenal impedance, most investigators have used RI. Others also use pulsatility index (PI), calculated as (peak systolic velocity - lowest diastolic velocity) / mean velocity.

For most renal examinations, sampling from at least three different sites within the kidney is adequate to obtain a mean intrarenal RI. The major problem with the technique has been technical error, either related to the technology used or the experience of the operator. However, the correct technique is not difficult to learn. 


\author{
Rl of normal kidneys
}

Adults

The renal vascular bed in a normal kidney has low impedance to blood flow, which is reflected by continuous forward flow in diastole. The range of normal renal resistance as measured by DU (RI determination) has been assessed by several investigators (Norris et al., 1984; Platt et al., 1989b; Gottlieb et al., 1989; Kim et al., 1992; Chen et al., 1993) and is summarized in Table 1 . The mean RI in normal adult human kidneys ranges from 0.57 to 0.64 . An RI value of 0.70 is usually regarded to be the upper limit in normal adult kidneys.

\title{
Children
}

Although an RI of 0.70 is accepted by most investigators as an upper level of normal in the adult population, more work needs to be done to establish accepted standards of normal and abnormal for paediatric renal Doppler waveforms. It has been shown that Doppler waveforms are likely to be age- dependent, particularly in infants (Gilbert et al., 1993; Keller et al., 1989; Keller et al., 1991; Shokeir et al. 1996b; Palmer et al., 1991). Bude et al. (1992) reported that the renal RI in healthy children is commonly above the upper limit in adults in the first year of life. The overal! trend shows a decrease of RI with age. From 4 years on, the likelihood is low ( $2 \%$ probability) that the RI is above 0.70 . Possible explanations for the higher RI in younger children are increased renal vascular resistance and decreased total renal blood flow in the infant kidney (Gilbert et al., 1993). Further research in the paediatric age group is needed to define the age at which the kidneys assume adult renal resistance level.

\section{RI of obstructed kidneys}

Platt et al. (1989) hypothesized that renal obstruction, like other states of increased vascular resistance, would produce a change in the Doppler waveform causing an increase in RI. In their initial report, the mean (SD) RI value of 14 obstructed kidneys was $0.77(0.04)$. Subsequently, data were presented confirming that ureteric obstruction produces a state of increased vascular resistance detectable by DU as an elevation of RI (Platt et al., 1989a; Platt et al., 1989b; Gottlieb et al,, 1989; Gibert et al., 1993; Palmer et al., 1991; Shokeir et al., 1996c) (table 1). 
Table 1: RI values of normal and obstructed adult human kidneys

\begin{tabular}{lccc}
\hline Reference & No. Pts. & Mean (SD) RI & Maximum RI \\
\hline Normal & 21 & $0.64(0.05)$ & 0.70 \\
Norris et al (1984) & 70 & $0.58(0.05)$ & 0.67 \\
Platt et al (1989b) & 15 & $0.58(0.04)$ & 0.66 \\
Gottlieb et al (1989) & 28 & $0.63(0.04)$ & - \\
Kim et al (1992) & 28 & $0.57(0.06)$ & 0.70 \\
Chen et al (1993) & & & \\
& & & \\
Obstructed & 14 & $0.77(0.04)$ & \\
Platt et al (1989a) & 4 & $0.75(0.06)$ & \\
Gottlieb et al (1989) & 38 & $0.77(0.05)$ & \\
Platt et al (1989b) & 10 & $0.75(0.04)$ & \\
Palmer et al (1991) & 7 & $0.83(0.10)$ & \\
Gilbert et al (1993) & 54 & $0.75(0.06)$ & \\
Shokeir et al (1996c) & & & \\
\hline
\end{tabular}

\section{Diagnostic accuracy}

Adults

In an effort to define a good discriminatory value to differentiate obstructive from non-obstructive dilatation, Platt et al. (1989b) reported on a large series of patients, including 70 with pyelocalyectasis, that allowed them to plot a receiver - operating curve that identified 0.70 to be an optimal discriminatory RI value. This threshold value achieved a sensitivity of $92 \%$, a specificity of $88 \%$, and an overall accuracy of $90 \%$ in diagnosing the presence or absence of obstruction in the adult population. Gottlieb et al. (1989) reported a sensitivity of $100 \%$ in a small group of adult patients. But conversely, Chen et al. (1993) reported a low sensitivity (53\%). The variation in the results could be attributed to the difference in the degree of urinary obstruction.

\section{Children}

In children, Gilbert et al. (1993) showed a sensitivity of $100 \%$ and a specificity of $85 \%$ when RI value of 0.70 was used to establish the diagnosis of obstruction against non-obstruction in 28 hydronephrotic paediatric kidneys. In a recent clinical study of 54 paediatric kidneys, we reported a sensitivity of $82 \%$, a specificity of $63 \%$, and an overall accuracy of $76 \%$ when an Rl of 0.70 was used as the critical value to predict obstruction (Shokeir et al., 1996c). 


\section{Causes of false-positive results}

\section{Abnormalities of pulse and blood pressure}

When an elevated RI is observed in a patient with presumed normal kidneys, the data should be correlated with the patient's heart rate and blood pressure. It was shown previously that heart rate and blood pressure at physiological extremes can alter the renal RI without renal pathology being present. Therefor, the level of these two variables should be known to interpret the renal RI. Significant hypotension and a low heart rate appear capable of producing an elevation of RI without a true change in renal vascular impedance (Mostbeck et al., 1990).

\section{Children}

As mentioned above, the $\mathrm{Rl}$ is higher in normal young children and infants than in adults (Gilbert et al., 1993; Keller et al., 1989; Keller et al., 1991; Shokeir et al., 1996b; Palmer et al., 1991; Bude et al., 1992). Consequently, specially in the very young patient, an RI value found to be elevated by the adult standard, may either be due to obstruction or may be normal for a young child. A normal RI (i.e.< 0.70 ) in this setting should be of value, arguing against obstruction in the setting of a dilated collecting system.

\section{Dehydration}

We have recently observed that the RI is $\geq 0.70$ in $54 \%$ of nonobstructed kidneys in fasting children (Shokeir et al., 1996b). After hydration, the RI regains its normal value; this observation addresses the importance of at least oral hydration for proper interpretation of Doppler studies.

\section{Renal medical diseases}

Previous investigators reported an elevation in renal vascular impedance with chronic hypertension (Norris et al., 1984), and acute renal failure (Wong et al., 1989). In 17 children with acute renal failure caused by the haemolytic - uraemic syndrome, Patriquin et al. (1989) found an elevated RI during the anuric- oliguric phase of this disease. Platt et al. (1989b) studied 50 patients with renal medical diseases and found elevated RI in half of these patients. Therefore, in the setting of known renal medical disease and pyelocalyectasis, an elevated RI could be due to the renal disease or obstruction, thus limiting the value of an abnormal RI in this particular situation. Again, a normal RI in this 
setting would still be helpful by arguing against the presence of obstruction.

\section{Causes of false- negative results}

\section{Acute obstruction}

The role of renal DU in the evaluation of acute renal obstruction has generated a vigorous commentary in the radiological Iiterature (Cronan et al., 1995; Platt et al., 1995; Urlich et al., 1995). To resolve this issue it is necessary to review the experimentally welldocumented blood flow changes associated with acute obstruction, that appear to follow a characteristic triphasic response (Vaughan et al., 1970). Immediately after obstruction, renal blood flow increases in response to rising ureteric pressure. This vascular response generally lasts less than $1.5-2$ hours, and is believed to be the result of preglomerular vasodilatation. Over the next $2-4$ hours, there is a gradual decrease in renal blood flow with continued elevation in the pelvic and ureteric pressures, presumably caused by postglomerular vasoconstriction. With obstruction lasting more than 3 - 5 hours, a third and chronic phase develops in which renal blood flow returns to baseline levels and then steadily declines. This vascular response is associated with decreasing intrapelvic and ureteric pressures and appears to be caused by preglomerular vasoconstriction. While experimental studies implicate vasodilators (prostagalndins) and vasoconstrictors (thromboxane, the renin - angiotensin system and endothelin) in these renovascular responses, the precise triggers, sequencing and mechanisms have yet to be defined (Urlich et al., 1995). Because, early in the time course of obstruction, vasoactive peptides cause renal blood flow to vary with time by producing opposite effects ( constriction and dilatation) in different segments of the glomerular vascular tree, it would be expected that before reaching the chronic and sustained phase of obstruction- induced vasoconstriction, RI measurements would reflect this variability and be inaccurate and non-diagnostic as an indicator of obstruction (Urlich et al., 1995).

\section{Mild obstruction}

Most investigators agree that marked partial ureteric obstruction results in an increase in intrarenal vascular resistance that is reflected as an elevation of $\mathrm{RI}$. The increase of vascular resistance in mild obstruction is still a matter of controversy and has generated a heated debate. While this concept has been supported by some (Ryan et al., 
1987), it has been questioned by others (Chen et al., 1993; Chevalier et al., 1986). Differences in results could be explained by the difference in the definition of mild obstruction. Although a normal Doppler study argues against significant obstruction, it does not imply that a ureter is free of any minimal regions of narrowing. Therefore, the renal Doppler examination alone does not suffice if precise anatomical information is required.

\section{Markedly dilated collecting system}

Platt et al. (1989b) also noted that markedly hydronephrotic kidneys did not show an increase in RI despite the presence of what was considered to be obvious urinary obstruction. This lack of response could be caused by a marked decrease in absolute blood flow in chronic high-grade obstruction, decreased filtration pressure produced by minimally functioning renal cortex or elevated compliance in a capacious dilated collecting system (Urlich et al., 1995).

\section{How to improve the diagnostic accuracy}

\section{Comparison to contralateral kidney without diuresis}

The accuracy of the discriminatory value of RI $(0.70)$ can be improved by evaluating the contralateral kidney, specially in acute obstruction in which the RI may not yet have exceeded the 0.70 limit. If there is a difference of $\geq 0.10$ between the obstructed and the contralateral kidney, this strengthens the diagnosis (Platt et a!., 1991). Comparison of the RI in the two kidneys increased the sensitivity from $57 \%$ to $71 \%$ in acute obstruction (Rodgers et al., 1992). The use of the obstructed to normal RI ratio (RIR) is also helpful. A ratio of 1.15 has been given by Urlich et al. (1995) as diagnostic of acute obstruction. Keller et al. (1991) used the RIR in 48 patients with unilateral obstruction and 34 normal controls, showing that with an RIR of $\geq 1.11$, the sensitivity for detecting obstruction was $77 \%$, while the specificity for excluding obstruction was $81 \%$. Obviously, comparison with the contralateral kidney is not suitable in patients with bilateral renal obstruction and in those with solitary kidneys. 


\section{Diuresis}

Ordorica et al. (1993) indicated that an increase of $\mathrm{Rl}$ of $\geq 15 \%$ after frusemide injection is diagnostic of obstruction. We have also shown that infusion of normal saline and administration of frusemide significantly increased the sensitivity, specificity, and overall accuracy of RI in the diagnosis of obstructed kidneys in children (Shokeir et al., $1996 \mathrm{c})$.

\section{Comparison to contralateral kidney with diuresis}

Palmer et al. (1991) investigated DU in children before and after administering intravenous frusemide, showing that it causes the RI to increase above baseline in obstructed kidneys and does not significantly affect the RI compared to baseline in normal and nonobstructed pyelocalyectatic kidneys. Bude et al. (1994) showed that infusion of normal saline and administration of frusemide significantly decreased the RI of non-obstructed renal units compared with baseline values. We have confirmed the results of Bude et al. in 14 children with normal upper urinary tracts (Shokeir et al., 1996b). In experimental studies, we showed that infusion of saline and frusemide causes the RI to decrease in non-obstructed kidneys and to increase in obstructed ones (Shokeir et al., 1996a; Shokeir et al., 1997a; Shokeir et al., 1997b). This divergent response could help identify obstruction and is better than a simple discriminatory value of RI. However, this approach is not applicable in solitary kidneys or bilateral obstruction.

\section{RI after relief of obstruction}

Platt et al. (1989a) reported a decrease of $\mathrm{RI}$ in nine of 10 adult patients 2-9 days after the relief of obstruction. Similarly, Ordorica et al. (1993) showed that, in all nine kidneys measured 3 months postoperatively the RI had decreased to $<0.75$. We also found a reversal of RI after relief of mild and severe degrees of obstruction in an experimental model (Shokeir et al., 1997b). On the other hand, Chen et al. (1993) measured RI in five adult patients after release of obstruction and found that in two the RI remained elevated. It would be interesting to determine the factors interfering with reversal of RI after the relief of obstruction, e. g. the age of the patient, type and duration of obstruction, together with the degree of vascular and parenchymal damage. 


\section{Prediction of recoverability of renal function}

We recently showed that recovery of renal function could not be predicted from the changes of RI before de-obstruction (Shokeir et al., 1997b). However, we noted that a reversal of a previously elevated RI could be used as an early indicator that recovery of renal function is likely. This could be applied clinically by monitoring RI before and after the temporary release of obstruction via percutaneous nephrostomy. In future, we may be able to couple DU with the administration of vasoactive drugs to detect a reversal of a high RI to predict recovery following corrective surgery (Vaughan et al., 1995).

\section{Relation with diuretic renography}

In 1985, Kass et al, used the drainage half-time $\left(\mathrm{T}^{1 / 2}\right)$ as an objective tool in the diagnosis of obstruction; $\mathrm{T}^{1 / 2}$ is defined as the interval necessary for half of the radioisotope in the renal collecting system to be eliminated after the administration of frusemide. Kidneys with a $\mathrm{T}^{1 / 2}$ of $<10 \mathrm{~min}$ are non-obstructed and those with $\mathrm{T}^{1 / 2}$ of $>20 \mathrm{~min}$ are obstructed. The overall accuracy of $\mathrm{T}^{1} 1 / 2$ in the diagnosis of obstruction approaches $90 \%$ (Kass et al., 1985).

Recent urological and radiological reports showed a good positive correlation between RI and $T_{1 / 2}$ (Gilbert et al., 1993; Shokeir et al., 1996a; Ordorica et al., 1993; Shokeir et al., 1996c). Thus a hydronephrotic kidney with a non-obstructed $\mathrm{T}_{1 / 2}$ on diuretic renography can be followed closely with surveillance renal DU, obviating the need for frequent isotope renal scintigraphy.

\section{Current clinical utility}

There is an expanding body of information which suggests that DU will be more often used to identify clinically significant obstructive uropathy. However, at present, DU does not suffice if precise anatomical information is required. Therefore, it cannot be used as a single modality for evaluating a patient with hydronephrosis. RI provides corroboration of the initial diuretic renogram and is considered as a non-invasive modality for monitoring the dilated collecting system under observation. Additionally, this method provides a non-invasive modality for monitoring patients after re-constructive surgery of the upper urinary tract. 


\section{Conclusions}

(i) Renal obstruction, like other states of increased vascular resistance, produces a change in the Doppler waveform causing elevation of RI. (ii) An RI value of 0.70 is accepted by most investigators to be the upper limit of normal in adult population. (iii) Renal outflow obstruction is not the only cause of elevation of RI. The RI value could exceed 0.70 without obstruction in children, particularly infants, and in patients with non-urological renal diseases. Moreover, dehydration, hypotension and a low heart rate appear capable to cause an elevation of RI without a true change in renal vascular impedance. However, a normal RI in these conditions is still a strong argument against obstruction. (iv) More work needs to be done to establish the role of RI in acute and in mild chronic renal outflow obstruction. (v) Diuretic DU is better than DU at baseline conditions. (vi) In cases of unilateral pyelocalyectasis, the use of RIR particularly after infusion of normal saline and administration of frusemide. significantly improves the diagnostic accuracy to identify obstruction. (vi) Experimentally, recovery of renal function could not be predicted from changes of RI before de-obstruction. However, reversal of a previously elevated RI could be used as an early indicator that recovery of renal function is likely. (viii) DU is a physiological investigation and is insufficient if precise anatomical details are required. Therefore, it could not be used alone to evaluate a patient with hydronephrosis. (ix) There is a good positive correlation between RI and $T_{1 / 2}$. Therefore, DU could be used for monitoring the dilated collecting systems under observation obviating the need for frequent radioisotope scintigraphy. 
1.2 Recoverability of renal function after relief of upper chronic partial urinary tract obstruction.

Ahmed A. Shokeir, Abraham P. Provoost and Rien J.M. Nijman

Urology \& Nephrology Center, Mansoura University, Mansoura,Egypt and Departments of Pediatric Surgery And Pediatric Urology, University Hospital, Erasmus University, Rotterdam, The Netherlands

Br. J. Urol. (in press) 

Obstructive uropathy with resultant hydronephrosis is the eventual outcome of many urologic disorders. Apart from accidental ligation or ureteric calculus, obstruction in humans is nearly always chronic and partial. The accurate prediction of recoverability of the kidney function after release of chronic partial obstruction is of great clinical value to the urologist and nephrologist. If restoration or improvement of renal function appears probable, surgical relief of obstruction may be indicated even though there has been a considerable initial loss of function. In patients whose kidney function is irreversibly damaged by the underlying obstructive process, it may be preferable to apply non-surgical management or total removal of the disordered system by nephrectomy. The aim of the present paper is to discuss the different factors that may potentially affect recoverability and to review the currently available tests that have been proposed to predict recovery of renal function upon relief of chronic partial obstruction.

\section{Factors that may affect recovery of renal function}

\section{Duration of obstruction}

The degree of recovery of renal function after release of unilateral ureteral obstruction correlates inversely with the duration of the obstruction (Vaughan et al., 1970; Ryan et al., 1978). In dogs there was rarely any return of renal function with release of unilateral complete ureteral obstruction after 40 days (Vaughan et al., 1971). However, the situation in humans is largely unknown. Return of function has been reported to occurr in clinical cases after more than $\mathbf{1 5 0}$ days of complete obstruction (Shapiro et al., 1976). The reason for the lack of correlation between the experimental data and the clinical situation is that the complete occlusion of the treter by ligation obtained in animals is not comparable to the incomplete occlusions that are more likely to occur in clinical types of hydronephrosis.

\section{Function of the contralateral kidney}

In the past, theories of renal counterbalance and renal atrophy of disuse have considerably affected the treatment options of long lasting unilateral ureteral obstruction. It was commonly believed that once hypertrophy of the contralateral healthy kidney has fully developed the damaged kidney would not regain its function (Hinman, 1943). Hydronephrotic atrophy was considered complete in 6 months, while compensatory hypertrophy was considered complete in 4-6 weeks (Hinman, 1943). Animal as well as human studies challenged this theory 
and now it is well established that recovery of even a poorly functioning kidney is possible despite compensatory hypertrophy of its mate. Nevertheless, the degree of recovery of the damaged kidney is influenced by the functional status of the contralateral kidney. Prominent recovery is seen under the stimulus of impaired function or removal of the opposite kidney (Kerr, 1956; Kerr, 1954; Provoost et al., 1981 ).

Schirmer and Hendricks (1969), studying metabolic aspects of unilateral hydronephrosis, reported that oxygen consumption in the obstructed kidney fell to $30 \%$ of control after 2 weeks of complete ureteral occlusion. If the obstruction was then released the oxygen consumption of the hydronephrotic tissue would recover to $70 \%$ of control. However, if the contralateral ureter was subsequently obstructed the recovery of the previously hydronephrotic kidney was further enhanced to $87 \%$ of control.

Age

Experimental studies in rats have indicated that age at the time of obstruction is an important factor determining the subsequent damage caused by ureteral obstruction. Provoost et al. (1989) reported that immature kidneys appeared to be more vulnerable to the damaging effects of a complete ureteral obstruction than adult ones. Furthermore, in a series of elegant experiments, Josephson and co-workers (Josephson, 1983; Stenberg et al., 1985; Josephson et al., 1987) showed that partial obstruction in the new born rat induced more severe damage compared to weaning or pubescent rats. Finally, it should be noted that in rats with congenital hydronephrosis, the mere presence of hydronephrosis not necessarily results in an impairment of glomerular filtration rate (GFR) (Provoost et al., 1990; Provoost et al., 1991).

In children, many urologists feel that the potential for recovery of what seems to be a severely damaged renal unit is great (Gillenwater, 1992). In a clinical study of 45 neonates with hydronephrosis, Koff and Campbell (1994) have demonstrated that even the most severely hydronephrotic functionally impaired kidneys had good potential for improvement. Bassiouny (1992) reported remarkable recovery of renal function in ten neonates with clinically palpable hydronephrotic nonvisualizing kidneys with differential renographic clearance less than $10 \%$. In the light of previous comments, the unpredictable recoverability of obstructed hydronephrotic kidneys in young children must govern all action. However, there are studies to the contrary which show clearly that kidneys with less than 10\% function may not perform in the long run and a reconstructive procedure may be part of a two-stage nephrectomy (Dlillon, 1998). 
Pyelo-lymphatic backflow

It has been postulated that preservation of renal function in hydronephrosis is augmented due to pyelo-lymphatic backflow. The reabsorption of renal pelvis urine into the lymphatics allows replacement of glomerular filtration to occur. It has been demonstrated that if the lymphatics alone are ligated, there is no necrosis, only a parenchymatous degeneration of the tubular cells. Ligation of both the lymphatics and the ureter produces severe renal damage with necrosis, and destruction may be seen in several days instead of several months (Gillenwater, 1992).

\section{Compliance of the ureter and renal pelvis}

If the ureter and renal pelvis are very compliant they would take the brunt of the back-pressure generated and protect the kidneys. However, if the ureter and pelvis are non-compliant, particularly if the pelvis is intrarenal, there will be significant calyceal dilatation and atrophy of the renal cortex.

\section{Other confounding factors:}

Return of function may well depend upon additional factors such as the presence or absence of infection, concurrent nephrotoxic medications, contrast materials, and other nephrotoxic agents. Moreover, the component of dysplasia in the kidney plays a major influence in recovery of renal function, particularly in children. If an obstructed kidney has a significant dysplastic component it will not regain its function significantly after release of obstruction.

\section{Methods of prediction of recoverability}

Excretory urography (IVU) and Grey-scale ultrasonography:

The IVU, though a primary diagnostic procedure, remains a qualitative examination and does not accurately indicate the functional mass of each kidney. IVU could not be used for prediction of recovery in obstructive uropathy. Many cases with non-visualising kidneys on IVU regained reasonable function after relief of obstruction in both children (Bassiouny, 1992) and adults (Lome et al., 1979).

Grey-scale ultrasonography is of great help in the evaluation of the degree of obstructive renal damage through assessment of the thickness of the renal parenchyma. Moreover, grey-scale ultrasonography can suspect renal medical diseases through the echogenicity of the renal 
parenchyma. Provided that there is no renal medical disease, the more the thickness of renal parenchyma, the better the recovery of the renal function after relief of obstruction (Belis et al., 1982).

Sequential ultrasound scans in the paediatric age group may reveal a good potential for recovery if there is renal growth in the interim. It has been shown clearly that if the kidneys stop growing or do not grow as expected, the situation may be slightly worse.

\section{Radioisotope renography:}

Radioisotope renal scan has been suggested as a method to assess the potential reversibility in functional impairment of renal tissue damaged by obstructive disease. Several agents have been used for this purpose, such as technetium-diethylenetriaminepentaacetic acid $\left({ }^{(\%)}\right.$ Tc-DTPA) (Bassiouny, 1992; Lome et al., 1979; Ransley et al., 1991), iodineorthoiodohippurate ( $\left.{ }^{131} \mathrm{I}-\mathrm{OIH}\right)$ (Shapiro et al., 1976; Kalika et al., 1981), and technetium-dimercapto-succinic acid ( $\left.{ }^{99 \mathrm{~m}} \mathrm{Tc}-\mathrm{DMSA}\right)$ (Chibber et al., 1981; Schelfhout et al., 1983). The results of various renal scans are conflicting because the different agents are handled differently by the kidney and thus measure different aspects of renal function. ${ }^{99 \mathrm{~m}} \mathrm{Tc}-\mathrm{DTPA}$ is eliminated by glomerular filtration only and has been used as an index of GFR. ${ }^{135} \mathrm{I}$-OIH is not only filtered but also secreted in renal tubular cells and measures renal blood flow rather than GFR, although a fair correlation with GFR is usually present. After injection, ${ }^{991 \mathrm{n}} \mathrm{Tc}-\mathrm{DMSA}$ accumulates in the cytoplasm of proximal tubular cells. Fifteen to $20 \%$ is eliminated in urine in 24 hours and it is used to assess functioning renal cortical tissue mass.

In early obstruction renal tubular function is altered minimally and tubular agents such as ${ }^{131} \mathrm{I}-\mathrm{OHH}$ and ${ }^{99 n} \mathrm{Tc}-\mathrm{DMSA}$ may be useful in documenting decreasing renal function (Kalika et al., 1981). However, in advanced stages of obstruction in which tubular function is altered maximally a glomerular agent, such as ${ }^{99^{m}} \mathrm{Tc}$-DTPA is the choice for evaluation of recoverable renal function. Sherman and Blaufox (1980) demonstrated that in patients with obstructive uropathy and nonvisualisation of the kidney with hippuran scan, even if the kidney does not take up any hippuran, a potential for functional recovery exists. ${ }^{9111}$ TcDTPA scintiscan images demonstrated residual function in advanced chronically obstructed kidneys, in which ${ }^{131} \mathrm{I}-\mathrm{O} H \mathrm{H},{ }^{99 \mathrm{~m}} \mathrm{Tc}-\mathrm{DMSA}$ and intravenous urography (IVU) all had been negative.

Recently, a new technetium-labelled physiologic analogue to ${ }^{131} \mathrm{l}$ $\mathrm{OIH}$, technetium-mercaptoacetyltriglycine $\left({ }^{9 \% \mathrm{~m}} \mathrm{Tc}^{\left.-\mathrm{MAG}_{3}\right)}\right.$ has been synthesised. Its biologic properties are similar to those of hippuran, but its physical properties are superior. This agent combines the advantages of high renal extraction with a proper energy emission for gamma camera. 
Both radio-pharmaceuticals are filtered by the glomeruli and secreted by the renal tubules. Studies in normal, poorly functioning, and transplanted kidneys have shown that ${ }^{9 \% \mathrm{n}} \mathrm{Tc}-\mathrm{MAG}_{3}$ can replace hippuran in renography and no side-effects have hitherto been reported (Hvid-Jacobson et al., 1990; Pavia et al., 1991; Muller-Suur et al., 1990). With these merits in mind, it becomes tempting to evaluate whether ${ }^{99 \mathrm{~m}} \mathrm{Tc}_{\mathrm{N}} \mathrm{MAG}_{3}$ scintigraphy can provide a sensitive tool for evaluation of renal function with the intent of prediction of potential functional recoverability in obstructive uropathy. The utility of this agent in this particular aspect has not been addressed in the literature.

Most investigators have used radionuclide quantification of renal function for assessment of renal recoverability. Basically, two approaches have been taken to quantify renal function via renography. One has been to perform absolute quantification, i.e. to obtain a discrete value of GFR or effective renal plasma flow (ERPF). There is an almost general agreement among urologists that if the relative renographic GFR or ERPF has fallen below $10 \%$ very little recovery of the kidney function can be expected after de-obstruction, whereas above $10 \%$ kidney function can improve considerably (Bassiouny, 1992; Lome et al., 1979; Ransley et al., 1991; Kalika et al., 1981; Chibber et al., 1981; Schelfhout et al., 1983). The better the radionuclide uptake, the better the recuperation after de-obstruction. In a recent experimental study using ${ }^{{ }^{99} \mathrm{~m}} \mathrm{Tc}-\mathrm{MAG}_{3}$ we have demonstrated that in dogs with a partial ureteral obstruction, resulting in an ERPF as low as $12 \%$ of the basal value, a good recovery is still possible upon removal of the obstruction (Shokeir et al., 1997b).

Renography may be misleading in neonates and young children with severe hydronephrosis because blood flow is diminished and the renal parenchyma is thin and displaced resulting in inaccurate calculation (Bassiouny, 1992). A poor renographic function alone does not justify nephrectomy in these patients. Therefore, the test should be used only to support surgical correction but not to justify nephrectomy. Whenever there is a question of potential renal function in children, additional diagnostic studies are indicated.

Kalika et (198I) al used a qualitative approach for predicting recoverability of renal function by comparing ${ }^{131} \mathrm{I}-\mathrm{OIH}$ renogram curves generated from the renal cortex and from the whole kidney. The results supported the hypothesis that when cortical curves appear more normal than total kidney curves there is a strong likelihood of post-operative improvement of renal function when the obstruction is relieved. Abnormal cortical curves are associated with a poor prognosis for renal functional improvement. Recoverability of kidney function in patients with acute obstruction was predicted in $89 \%$ and in those with chronic obstruction in $96 \%$. The overall accuracy for acute and chronic obstruction was $94 \%$ (Kalika et al., 1981). However, because of the occasional patient who may 
have improvement despite a negative prediction, Kalika et al. (1981) recommended that the test is better suited at present towards choosing patients in whom a possibility of surgical benefit exists.

Percutaneous nephrostomy $(P C N)$

A PCN performed before surgical correction can provide information about the potential to recover of the affected kidney. Ransley et al. (1991) studied newborns with ureteropelvic junction obstruction and poor renal function by insertion of a pigtail nephrostomy tube. They underwent a repeat ${ }^{99 \mathrm{~m}} \mathrm{Tc}$-DTPA scan after 3 weeks of drainage, and proceeded to either nephrectomy (if function is less than 10\%) or pyeloplasty (if function is more than $10 \%$ ). One third of these patients showed useful functional recovery. Therefore, they recommended that nephrectomy should not be performed without a period of pigtail catheter drainage (Ransely et al., 1991). Nevertheless, the same authors have recently changed their strategy. They no longer use nephrostomy drainage and proceed directly to nephrectomy if the ${ }^{99 m} \mathrm{Tc}-\mathrm{MAG}_{3}$ GFR is less than 10\% (Dhillon, 1998).

Gillenwater (1992) stated that the best method to determine absolute degree of injury and recoverability is by temporarily relieving the obstruction with a nephrostomy tube and following renal function by creatinine clearance. However, he did not indicate the level of creatinine clearance above which obstruction should be corrected. Cronan (1991) advised to allow the renal unit sufficient time for recovery after placement of PCN. Up to 8 weeks is necessary before the kidney has established its new baseline level of function. On the other hand, Bassiouny (1992) did not recommend nephrostomy in neonates with poor renal function because it may be difficult to perform in neonates and eventually introduces infection, causes retraction of the renal pelvis and additional renal damage, and increases the risk of complications at the time of pyeloplasty with more difficult surgery.

\section{Surgical Exploration}

Bassiouny (1992) has recently reported his experience in the management of 10 neonates who presented with non-visualising hydronephrotic kidneys due to ureteropelvic junction obstruction with a differential ${ }^{1 \% m}$ Tc-DTPA uptake less than $10 \%$. He recommended that the decision to remove or preserve a kidney in a neonate should be made at surgery after prior confirmation of the presence of obstruction. At operation the parenchyma usually appears thin and stretched over a distended tense renal pelvis (Bassiouny, 1992). An assessment should be made of the overall bulk of the stretched-out renal parenchyma rather than 
its thickness since decompression may be rewarded by retum of significant kidney function (Johnston et al., 1977). Using the policy of immediate surgical repair after confirmation of the diagnosis, Bassiouny (1992) reported remarkable recovery of renal function (more than $100 \%$ on renal scintigraphy, albeit from low basal levels) in all his patients.

\section{Enzymuria}

Huland et al. (1988) put the theory that hydronephrotic atrophy in adult rats after different grades of stable partial ureteral obstruction develops in 2 phases: a destructive phase followed by a steady-state phase. They showed that relief of obstruction in the destructive phase, but not in the steady-state phase, was able to improve or prevent hydronephrotic atrophy. Since the duration of the ureteral obstruction in humans is usually not known, the urinary enzymes were studied in rats after partial unilateral ureterat obstruction to identify the destructive phase. They chose two markers of tubular damage, the lysosomal enzyme $\mathrm{N}$-acetylglucosaminidase (NAG) and the brush border enzyme gamma-glutamytransferase (gamma-GT). The NAG concentration, but not so much gamma-GT concentration, was higher in the urine of the obstructed kidney than in the urine of the contralateral control kidney, in the first two weeks after obstruction, and then returned to nomal. These observations lead to the conclusion that the destructive phase after unilateral ureteral obstruction can be identified by the appearance of high urinary tubular Jysosomal enzyme content. The clinical implication is that the timing of relief of asymptomatic stable partial ureteral obstruction of unknown duration can be based on the concentration of urinary lysosomal enzymes.

Clinical studies in children (Carr et al.. 1994) as well as in adults (Tataranni et al., 1987) are promising, indicating that urinary enzymes may be helpful in the diagnosis of upper urinary tract obstruction and in identifying the proper timing of its relief. However, urinary enzymes may be elevated in conditions other than urinary obstruction, among them tubular damage due to nephrotoxic drugs, hypertension, diabetes, and acute tubular necrosis (Huland et al., 1998). Moreover, high urinary enzymes was noted in patients with acute urinary tract infection (Tataranni et al., 1987). More work needs to be done to determine the clinical usefulness of urinary enzymes in prediction of recoverability of renal function after release of obstruction.

\section{Transforming growth factor-beta (TGF-b)}

TGF-b is one of the growth factors which are elaborated by the kidney and have been implicated in nephrogenesis. In the kidney, TGF-b affects mesenchymal differentiation and promotes extracellular matrix 
formation which results in collagen synthesis. Specifically in the urinary tract, TGF-b is elevated in the kidney after induced obstruction (Walton et al., 1992). A high level of TGF-b expression correlates with muscle hypertrophy and increased collagen deposition which remodel the renal pelvis in response to obstruction. The lower level of TGF-b expression in chronic obstruction may reflect a limit of re-modeling once a steady state has been achieved.

Seremetis and Maizels (1996) have recently demonstrated that there is an increase of TGF-b mRNA expression in the renal pelvis following clinical and experimental ureteropelvic junction obstruction. They have also shown that high level of TGF-b mRNA expression correlated significantly with good clinical outcome. Other studies are invited to confirm these observations and to determine the correlation between the level of TGF-b and recoverability of renal function.

\section{Doppler ultrasonography}

Resistive index (RI) obtained during Doppler ultrasonography (DU) has been recently described by Platt et al. (1998a). The non-invasive nature of measurement of renal RI gives it considerable appeal in its potential application to patients with obstructive uropathy. We have recently demonstrated in an experimental study that recovery of the renal function could not be predicted from the changes of Rl before deobstruction (Shokeir et al., 1997b). However, we have revealed that a reversal of a previously elevated RI could be used as an early indicator that recovery of renal function is likely. This could be clinically applied by monitoring $\mathrm{Rl}$ before and after temporary release of obstruction via percutaneous nephrostomy. In the future, we may be able to couple DU with the administration of vasoactive drugs to look for a reversal of a high RI to predict recovery following corrective surgery (Vaughan, 1998).

\section{Other suggested methods}

Renal Biopsy

To our knowledge there are only three studies to document histological changes in children with ureteropelvic junction obstruction (Steinhardt et al., 1998; Krueger et al.,1980; Elder et al., 1995). In severe cases ureteropelvic junction obstruction may cause substantial renal damage. Steinhardt et al. (1989) studied 20 patients undergoing nephrectomy for severe obstruction of whom $75 \%$ had interstitial fibrosis with inflammation, $70 \%$ had glomerulosclerosis with inflammation, $30 \%$ had medullary dysplasia and 15\% had glomerular cystic changes. Krueger et al. (1980) previously showed that renal biopsy was normal in 7 of 13 children $(54 \%)$ undergoing pyeloplasty. In that series 2 of 4 patients with a 
half- time drainage less than 10 minutes had an abnormal biopsy but 4 of 6 with a half-time drainage greater than 30 minutes had a normal renal biopsy. Elder et al.(1995) retrospectively analyzed the renal biopsy obtained during pyeloplasty in 55 children with a mean age of 4.9 years (range: 4 days to 19 years). Histological changes were compared to the preoperative differential renal function. Histological changes were graded on a scale of 1 to 5 . Differential function on diuretic renography correlated with histological grade since kidneys with normal histology had a higher differential renal function than abnormal kidneys. However, from those kidneys with differential function greater than $40 \%, 21 \%$ had a histological grade 3 or 4 on renal biopsy. Conversely, from those kidneys with differential function less than $40 \%, 33 \%$ were normal or had minimal histological changes. Thus, in about $25 \%$ of children with ureteropelvic junction obstruction there is a disparity between preoperative differential renal function computed during diuretic renography and the findings on renal biopsy.

To the best of our knowledge, the correlation between the different degrees of histological changes during obstructive uropathy has never been correlated to the recoverability of renal function after relief of obstruction. Studies of such a subject may help urologist to predict the degree of recoverability of renal function after relief of obstruction according to information gained via preoperative percutaneous renal biopsy.

\section{Estimation of renal parenchymal volume}

One of the arbitrary criteria used to indicate whether repair of obstructive uropathy is warranted is the measurement of the parenchymal thickness by ultrasonography. It has been estimated that the presence of a centimeter or more of parenchyma is a good prognostic parameter (Belis et al., 1982). In hydronephrotic kidneys, however, the parenchymal thickness is irregular and its measurement at one or more points does not reflect the true amount of the remaining nephrons. Therefore, we suppose that measurement of the whole volume of renal parenchyma by ultrasound, CT, or MRI may be beneficial in the context of prediction of recoverability of renal function in obstructive uropathy.

Preoperative percutaneous renal biopsy and estimation of the volume of renal mass provide both qualitative and quantitative information of the renal parenchyma and may be a good combination in prediction of recoverability of renal function.

\section{Renal finctional reserve}

The concept of renal functional reserve has been recently introduced to express the extent to which kidney can respond to a metabolic or haemodynamic overload. The idea of the test is to measure 
the selective renal function at basal condition by radioisotope renography; then the kidney is put under a stress condition by induction of diuresis, an injection of dopamine and an amino acid-load. The selective renal function is measured again under the stress condition. The renal functional reserve is determined by subtracting the renal function at basal condition from that under stress. The details of the test was previously described (Shokeir et al., 1994). Some functional reserve can always be detected this way, even in patients with significant advanced renal damage. Whether the estimated functional reserve correlates with the potential of recoverability after deobstructing a hydronephrotic kidney is difficult to ascertain at present.

\section{Conclusions}

1. An IVU is not very suitable to predict recovery of renal function in obstructive uropathy.

2. Most investigators agree that in the adult population, a kidney with a ${ }^{2} \%$ TC-DTPA GFR of less than $10 \%$ of the total normal GFR (approximately $10 \mathrm{ml} / \mathrm{min}$ ) is considered not salvable.

3. Renograms may be misleading in neonates and young children. Therefore, they should be used to support the decision for surgical correction. The decision of nephrectomy should not be solely based on renograms, but other factors should also be considered.

4. It is difficult to differentiate the unsalvable from potentially salvable kidneys when using a single test only. Under such circumstances, combining different tests may be helpful to reach a proper decision.

5. Extensive work needs to be done to establish the clinical usefulness of the new non-invasive predictors of recoverability of renal functional, such as, urinary NAG excretion, renal TGF-b expression, and the RI.

6. Correlations between recoverability of renal function and histological changes secondary to obstruction, like renal mass as determined by ultrasound, CT, or MRI, and renal functional reserve need to be studied. 


\subsection{Scope of the thesis}

The aim of this study was to determine the role of renal resistive index $(\mathrm{RI})$ in the diagnosis of obstructive uropathy. We developed a new canine model for induction of partial ureteric obstruction (Chapter 2) and we used this model to study $\mathrm{Rl}$ in 3 experimental studies. In the first experimental study (Chapter 3), we investigated the effect of intravenous normal saline fluid load, with and without frusemide, upon the renal RI of obstructed and non-obstructed kidneys. In the second experimental study (Chapter 4), we studied the changes in renal RI and renal function with time during different grades of partial unilateral ureteric obstruction, and we determined the correlation between the ultrasonographic and renographic findings. In the third experimental study (Chapter 5), we studied the changes in renal RI before and after release of different grades of partial unilateral ureteric obstruction.

After the encouraging results of the experimental work, we carried out 3 clinical studies in children. In the first clinical study (Chapter 6), we studied the effect on RI of fasting, intravenous infusion of normal saline, and administration of frusemide in children with normal upper urinary tracts. In the second clinical study (Chapter 7), we investigated the effect of hyperhydration with normal saline and frusemide on renal RI in children with unequivocal obstructive uropathy (half-time drainage, $T_{1 / 2}>20 \mathrm{~min}$.). In the third clinical study (Chapter 8 ), we studied the effect of hyperhydration with normal saline and frusemide on the renal $\mathrm{RI}$ in children with equivocal obstructive uropathy $\left(\mathrm{T}_{1 / 2} 10-20 \mathrm{~min}\right.$.). 

Chapter 2

Partial ureteral obstruction: a new variable and reversible canine experimental model

Almmed A. Shokeir

Urology \& Nephrology Center, Mansoura University, Mansoura, Egypt.

Urology (1995) 45: 953-957 



\section{Abstract}

Objectives

To develop a new experimental model of partial ureteral obstruction that is simple, reliable, variable, and reversible.

\section{Methods}

A $6 \mathrm{~F}$ ureteral catheter was inserted into the left ureteral orifice and was cut $2 \mathrm{~cm}$ distal to the orifice. The most distal part of the ureter was ligated around the catheter. The catheter lumen was partially obstructed by insertion of stylets of three different diameters. The catheter and the stylet were fixed inside the bladder. The model was tested in 12 dogs, which were stratified into three groups according to the diameter of the obstructing stylet. All the dogs were subjected to intravenous urography (IVU) and technetium-99m-mercaptoacetyltriglycine $\left({ }^{9 \%} \mathrm{Tc}_{\mathrm{M}} \mathrm{MAG}_{3}\right)$ renal scan at 2 and 4 weeks following induction of partial ureteral obstruction.

Results

Three different grades of hydroureteronephrosis were obtained according to the diameter of the obstructing stylet. The IVU and ${ }^{99 \mathrm{~m}} \mathrm{Tc}-$ $\mathrm{MAG}_{3}$ renal scan studies were repeated at 2 and 4 weeks following removal of the ureteral catheter and ureteroneocystostomy and showed marked improvement in the configuration and function of the corresponding renoureteral units.

\section{Conclusions:}

Our experimental model fulfills the requirements for a useful model of partial ureteral obstruction, namely, simplicity, reliability, variability, and reversibility.

\section{Introduction}

Obstructive uropahty with resultant hydronephrosis is the eventual outcome of most urologic diseases. However, the mechanisms by which renal failure occurs as a result of obstruction and recoverability of renal function after release of obstruction are not fully recognized. The development of an experimental model that closely mimics partial ureteral obstruction would allow investigation of the 
pathophysiologic change in ureteral and renal function. It also allows evaluation and standardization of the clinical tests used to measure the degree of obstruction. Moreover, a reversible model would allow study of recoverability of kidney function after relief of obstructive uropathy. The useful model has to: (I) provide an accurate and persistent degree of partial ureteral obstruction, (2) enjoy a versatile degree of obstruction, and (3) result in reliable and reversible ureteral obstruction. Although several models for the production of experimental hydronephrosis have been reported (Boyarsky and Martinez, 1964; Deluca et al., 1961; Ulm and Miller, 1962; Guze and Q'shea, 1985; Algood et al., 1983; Holmund and Hassler, 1965; Rayan and Fitzpatrick, 1987), as yet no model fulfilling all of those criteria has been developed.

The objective of this study is to develop a new canine experimental model of partial ureteral obstruction that is simple. reliable, variable, and reversible.

\section{Material and Methods}

\section{Experimental animals}

Twelve male mongrel dogs weighing 15 to $20 \mathrm{~kg}$. were used. The procedures were carried out under general anesthesia using thiopental sodium $(10 \mathrm{mg} / \mathrm{kg})$ with endotracheal intubation and mechanical ventilation.

\section{Preoperative assessment}

All dogs were subjected to ascending cystography, intravenous urography (IVU), and technetium - 99m-mercaptoacetyltriglycine $\left.{ }^{(2) m_{1}} \mathrm{Tc}_{\mathrm{C}} \mathrm{MAG}_{3}\right)$ and renal scan before start of the experiments. Dogs that showed primary vesicoureteral reflux or abnormality in the configuration or function of one of their kidneys were excluded from the study.

\section{Technique of partial ureteral obstruction}

The urinary bladder was exposed via a midline transperitoneal incision. The bladder was opened and a $6 \mathrm{~F}$ ureteral catheter was inserted into the left ureteral orifice. The catheter was cut $2 \mathrm{~cm}$ distal to the left ureteral orifice. The most distal part of the ureter was ligated with 3-0 silk ligature around the previously inserted ureteral catheter, preventing passage of urine around the outer surface of the catheter. The ureteral catheter was partially obstructed by insertion of stylets of 
different diameters inside its lumen. The ureteral catheter and the stylet were fixed to the bladder mucosa by 3-0 silk sutures (Fig. 1). The ureteral catheter used was semirigid plastic tube (Porges, 24200, Sarlat, France). Its external diameter was $1.8 \mathrm{~mm}$ and the internal diameter was $0.96 \mathrm{~mm}$. Plastic stylets of three different sizes were left inside the lumen: size I with $0.40 \mathrm{~mm}$ diameter (stylet of $4 \mathrm{~F}$ ureteral catheter), size II with $0.58 \mathrm{~mm}$ diameter (stylet of $5 \mathrm{~F}$ ureteral catheter), and size III with $0.78 \mathrm{~mm}$ diameter (stylet of $6 \mathrm{~F}$ ureteral catheter). In all the dogs, the right renoureteral unit was left undisturbed, serving as a control.

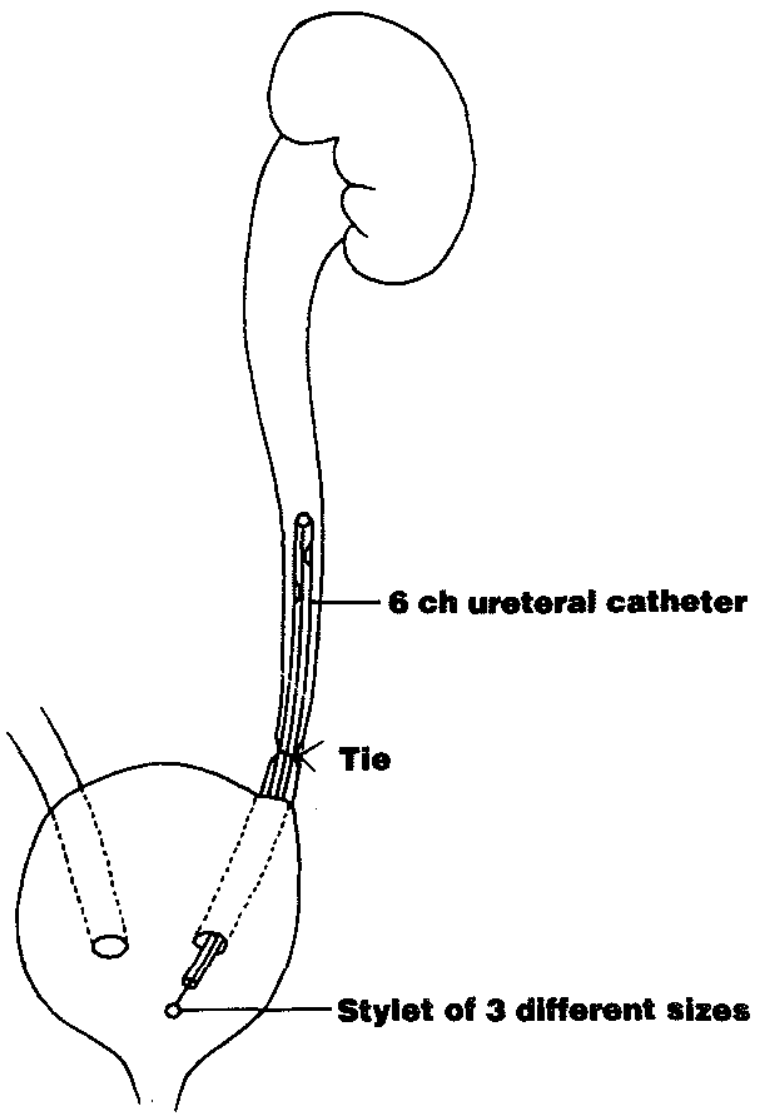

Figure I: A diagram representing the technique used to induce partial variable ureteral obstruction. 


\section{Experimental groups}

The 12 dogs were stratified into three groups ( 4 dogs each) according to the size of the stylets. Stylet sizes I, II, and III were used for groups I, II and III, respectively. The areas of the free space between the walls of the catheter and the stylets were $0.598,0.460$, and 0.246 $\mathrm{mm}^{2}$ for groups I, Il and III, respectively (Table I). These free spaces will allow passage of different amounts of urine, creating different degrees of hydroureteronephrosis.

Table I: Stylet size and arca of the free space between the wall of the ureteral catheter and the stylets

\begin{tabular}{lcc}
\hline Group & Stylet diameter $(\mathrm{mm})$ & Area of free space $\left(\mathrm{mm}^{2}\right)$ \\
\hline Group I & 0.40 (size I) & 0.598 \\
Group II & 0.58 (size II) & 0.460 \\
Group III & 0.78 (size III) & 0.246 \\
\hline
\end{tabular}

Postoperative assessment

All 12 dogs were subjected to IVU and ${ }^{9911}$ Tc-MAG3 renal scans at 2 and 4 weeks following induction of partial ureteral obstruction. After 4 weeks, the dogs were reopened, the left ureter was severed at its most distal end, the ureteral catheter was removed, and Lich-Gregoir ureteroneocystostomy (Lich et al., 1961; Gregoir and Van Regemorter, 1964) was carried out. No stents or catheters were left. IVU and ${ }^{9{ }^{2} \mathrm{~m}}$ Tc-MAG3 renal scan studies were repeated at 2 and 4 weeks following ureteroneocystostomy. In all dogs urine samples for culture were obtained from both ureters just before induction of partial ureteral obstruction and before ureteroneocystostomy. Both procedures of induction and relief of ureteral obstruction were covered by antibiotic prophylaxis (amoxicillin $500 \mathrm{mg}$ twice daily for 3 days).

\section{Results}

All animals survived the experiments, and there were no complications observed due to either the indwelling ureteral catheter or the ureteroneocystostomy. All urine samples obtained for culture were free of infection. 
Intravenous urography studies

Preoperative IVU demonstrated the relatively sinall size of the canine renal pelvis (Fig. 2A). After induction of partial ureteral obstruction, IVU demonstrated progressive hydroureteronephrosis that was increasing in severity as the degree of obstruction became marked (Fig. 2B, C, and D). Significant improvement in the configuration of the corresponding renoureteral unit was achieved in all dogs 4 weeks after ureteroneocystostomy (Fig. 3).
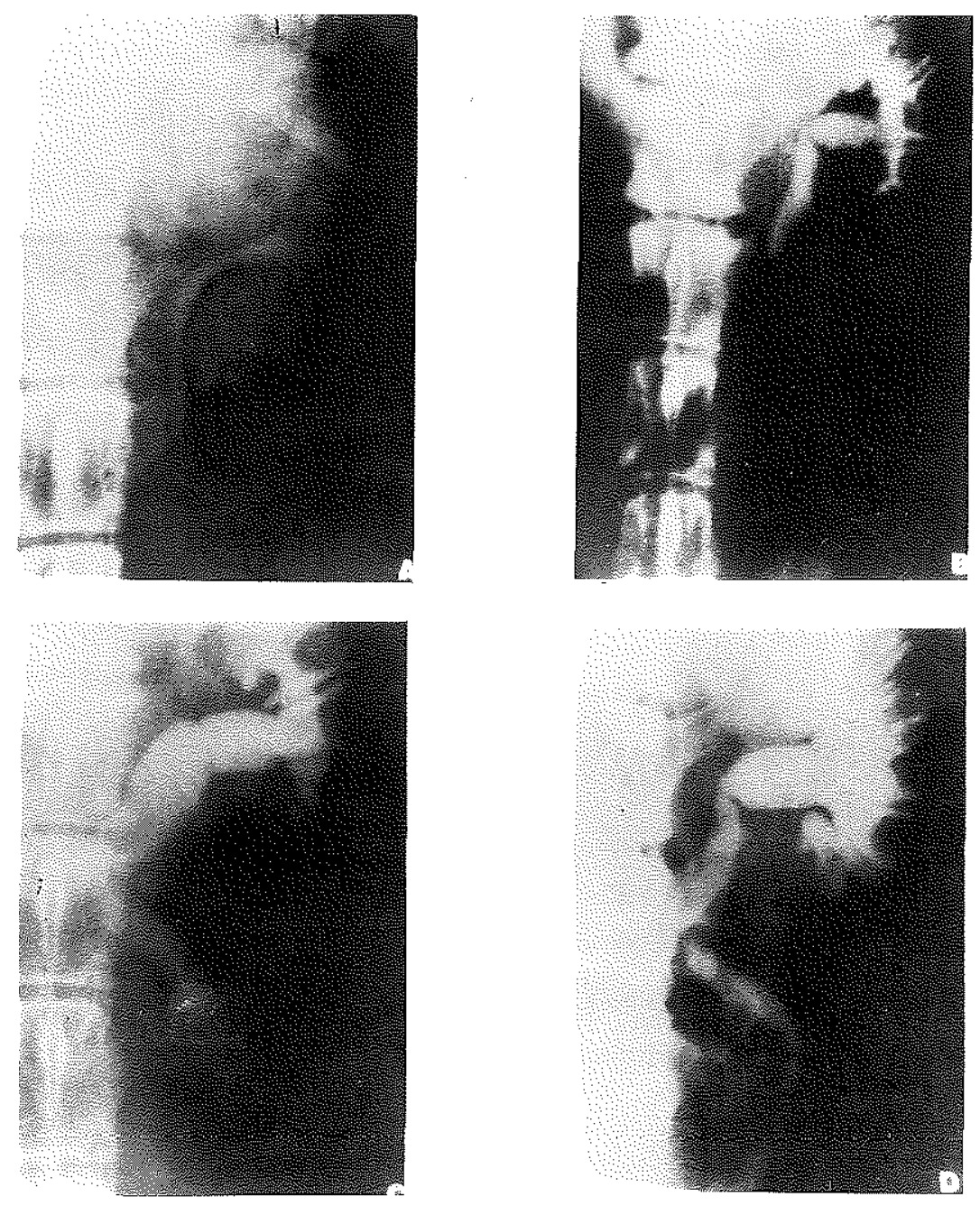

Figure 2: Intravenous urogram showing left renal pelvis and upper ureter of normal kidney (A), grade 1 hydroureteronephrosis (B), grade 2 hydroureteronephrosis (C), and grade 3 hydroureteronephrosis (D). 


\section{Radioisotope studies}

A significant diminution in renographic clearance of the left kidney was observed in all groups 4 weeks following induction of partial ureteral obstruction (Student's $t$ test, $P<0.003,0.0005$, and 0.00001 for groups I,II, and III, respectively). Comparison of selective renographic clearance of corresponding renal units before and after ureteroneocystostomy revealed significant improvement (Student's $t$ test, $p<0.028,0.01,0.0046$ for groups I, II and III, respectively) (Fig. 4). After relief of ureteral obstruction, the kidneys regained $84.2 \%, 66.7 \%$, and $58.2 \%$ of their basal function in groups I, II, and III, respectively. The renogaphic clearance of the left kidney of individual animals before induction of ureteral obstruction, 4 weeks after the obstruction and 4 weeks after the obstruction was removed are given in Fig.5.
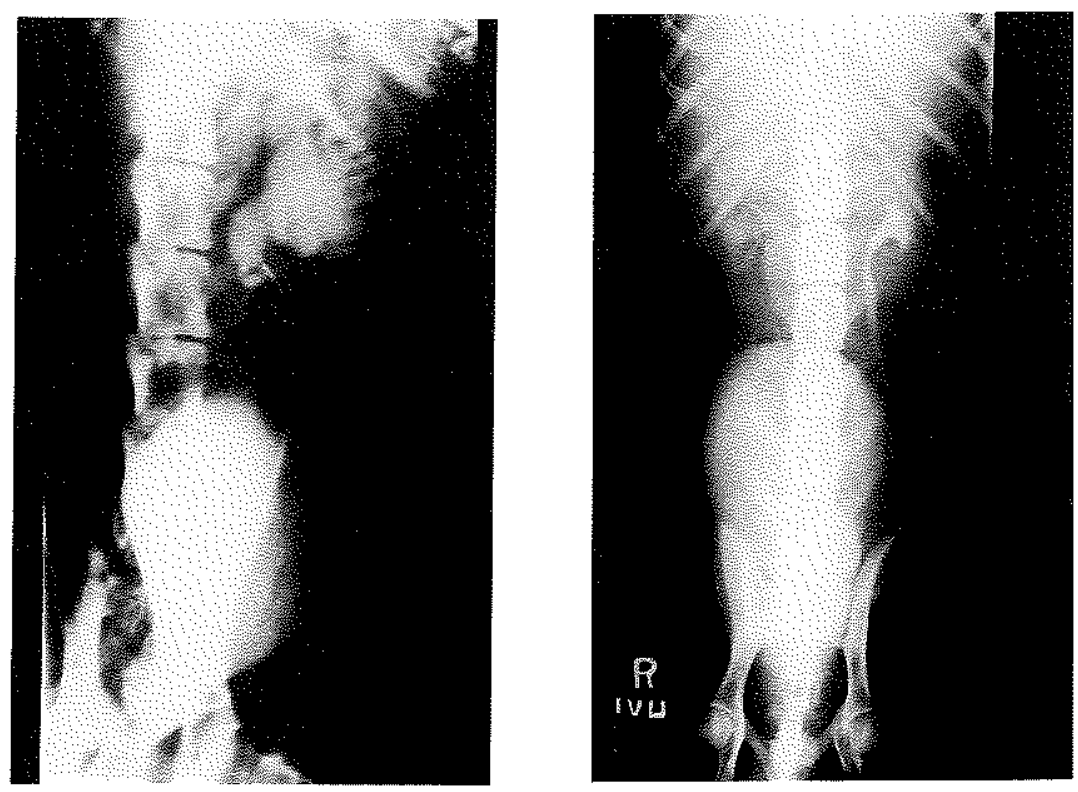

Figure 3: Intravenous urogram of one of the group III dogs. (A) left hydroureteronephrosis 4 weeks after induction of partial ureteral obstruction. (B) disappearance of left hydronephrosis 4 weeks after ureteroneocystostomy.

\section{Comment}

The experimental model of partial ureteral obstruction should provide a measurable degree of obstruction, which should persist for the duration of the experiment. It should also allow variation in the degree 
of obstruction. Unless the method of producing hydronephrosis is reversible, recoverability of kidney function after relief of obstructive uropathy cannot be investigated.

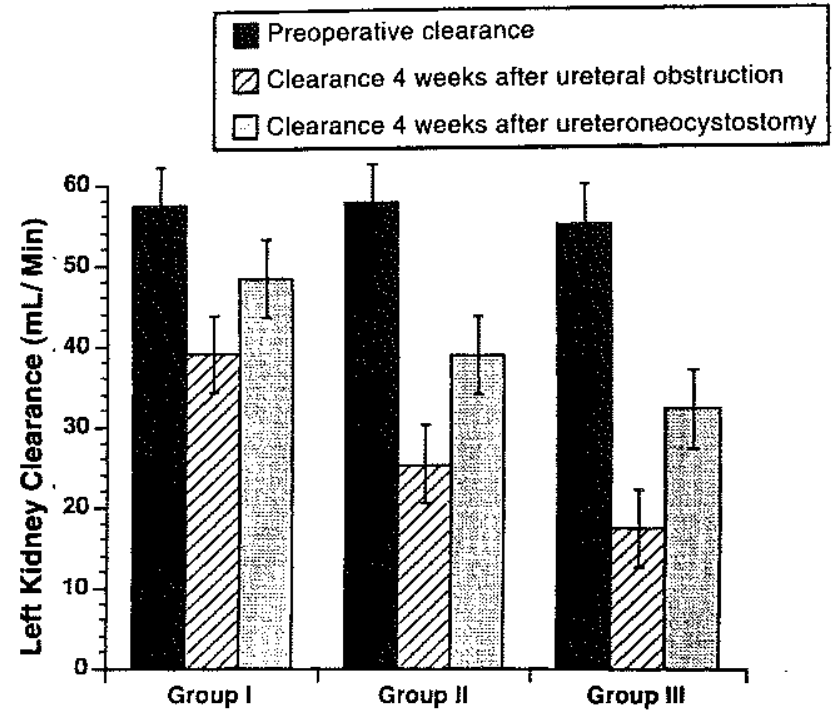

Figure 4: ${ }^{3 / 1 \mathrm{~m}} \mathrm{Tc}_{\mathrm{c}} \mathrm{MAG}_{3}$ clearance of the left kidney.

Several experimental models have been designed to create partial ureteral obstruction. Of these are partial ligation of the ureter with sutures or with aluminum and cellophane bands (Deluca et al., 1961), embedding the ureter into the psoas muscle (Ulm and Miller, 1962), beta irradiation of the ureter (Guze and Q'shea, 1958), and insertion of artificial calculus via a vesicostomy (Algood et al., 1986), or a ureterostomy (Holmund and Hassler, 1965). However, none of these models provides variable or reversible hydronephrosis. Recently, Ryan and Fitzpatrick (1987) introduced a new variable canine experimental model for partial ureteral obstruction by insertion of a $2-\mathrm{cm}$ obstructing stent into the midureter, and the degree of obstruction was varied by altering the internal diameter of the stents. Although this technique is variable, the authors did not report on reversal of hydronephrosis.

The advantage of our experimental model are numerous. The exact grade of ureteral obstruction can be achieved. Moreover, ureteral occlusion is reliably reversible. Unlike the Ryan and Fitzpatrick model (1987), our model does not need temporary proximal urinary diversion via a percutaneous nephrostomy tube. The presence of ureteral catheter in a closed urinary tract avoids the problems of infection and pulling of the catheter by the dogs. The use of Lich-Gregoir ureteroneocystostomy for reversal of hydronephrosis is simple, reliable, and provides both 

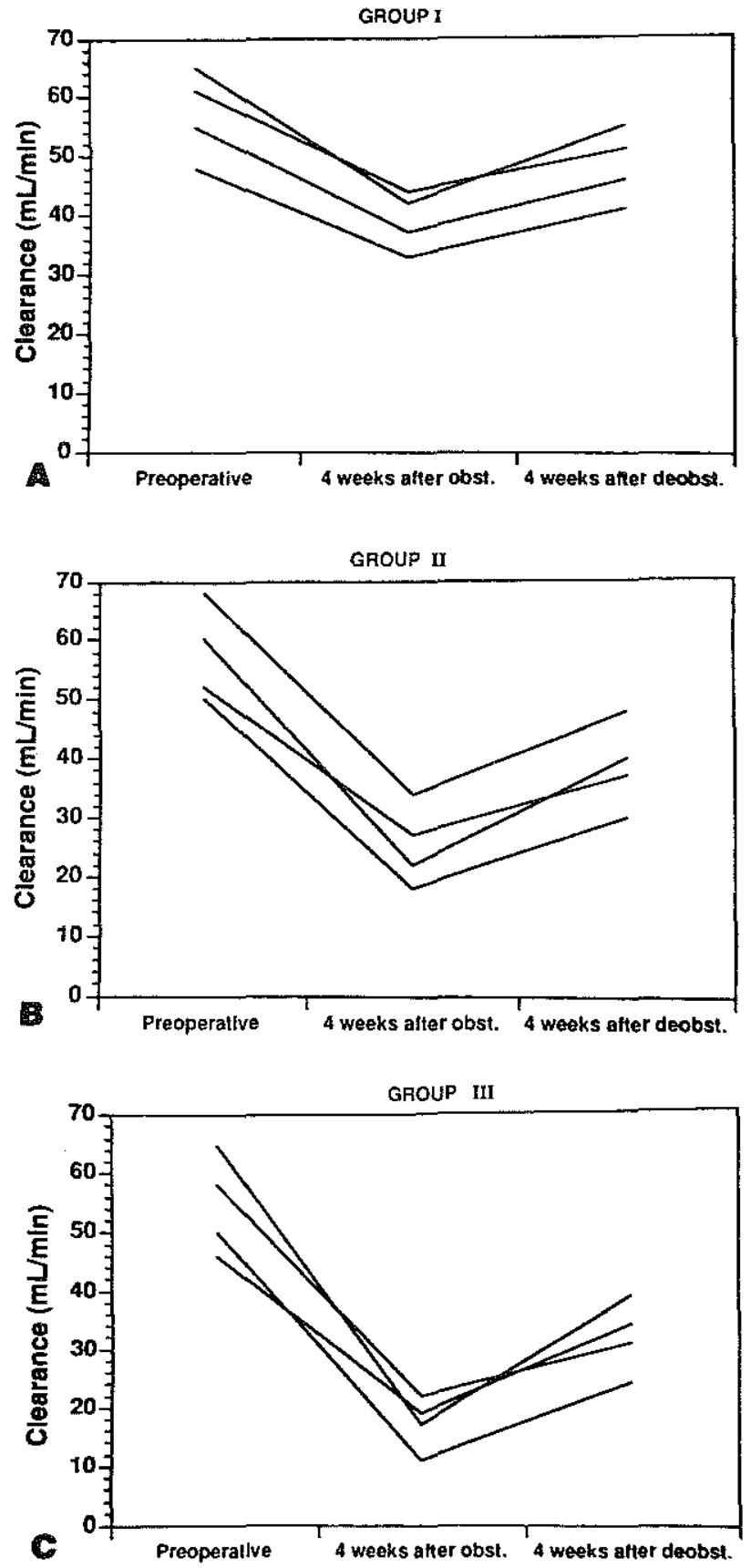

Fig. 5: Renographic clearance of the left kidney for different groups. (A) group I; (B) group II; (C) group III. 
reflux prevention and absence of obstruction (Marberger et al., 1978; Mjalmas et al., 1992).

In this study, a significant diminution in renographic clearance of the corresponding renal units was observed in all animals 4 weeks following induction of partial ureteral obstruction. The use of narrower catheters for longer duration is likely to result in more marked diminution of renographic clearance values.

The principle of our model could be applied with the ureteral catheter brought out on the back of the dog in a subcutaneous tunnel at a point that the dog is unable to reach. Under such circumstances, the degree of hydronephrosis could be changed in the same dog and urine samples could be obtained from each kidney.

The ureteral catheter and the stylets could also be replaced by manufacturing a special ureteral stent with fixed external diameter and with different internal diameters allowing drainage of a variable amount of urine and creating different degrees of hydronephrosis.

In summary, our experimental model fulfills the requirements for a useful model of partial ureteral obstruction, namely, simplicity, reliability, variability, and reversibility. 

Chapter 3

Partial ureteral obstruction: effect of intravenous normal saline and furosemide upon the renal resistive index

Almed A. Shokeir, Rien J.M. Nijman, Mohamed El-Azab, and Abraham P. Provoost

Urology and Nephrology Center, Mansoura University, Mansoura, Egypt, and Departments of Pediatric Urology and Pediatric Surgery, Erasmus University, Rotterdam, The Netherlands

J. Urol. (1997) 157: 1074-1077 



\begin{abstract}
Objectives

To investigate the effect of intravenous normal saline fluid load, with and without furosemide, upon the renal resistive index (RI) of obstructed and non-obstructed kidneys.
\end{abstract}

\title{
Methods
}

Right partial ureteral obstruction was induced in 10 dogs, Grade 1 (mild) obstruction was performed in 5 dogs (group A), and grade 3 (severe) obstruction was carried out to the remaining 5 dogs (group B). Evaluation by Doppler ultrasonography was performed before induction of ureteral obstruction and by the end of the eighth week of obstruction. Every obstructed animal was subjected to bilateral renal Doppler ultrasonography 3 times in one setting:1) before infusion of normal saline, 2) 30-60 minutes after intravenous infusion of normal saline (15 $\mathrm{ml} . / \mathrm{kg}$.) given in a rate of $1 \mathrm{ml} / \mathrm{kg} . / \mathrm{min}$, and 3) 10 minutes after admission of furosemide ( $1 \mathrm{mg} . / \mathrm{kg}$.).

\section{Results:}

After induction of right partial ureteral obstruction, there was a significant increase of the RI of the right kidney and a significant decrease of the RI of the left kidney compared to baseline RI in both groups. Infusion of normal saline and administration of furosemide caused a further significant increase of the RI of the obstructed kidney and a further significant decrease of the RI in non-obstructed kidney in both groups.

\section{Conclusions}

In unilateral partial ureteral obstruction, addition of intravenous normal saline and furosemide cause the RI to increase in obstructed kidney and to decrease in non-obstructed kidney. Such a divergent response may be useful for the development of a pharmacologically challenged Doppler examination to diagnose better potentially obstructed kidneys.

\section{Introduction}

The diagnosis of urinary tract obstruction is a difficult and perplexing problem, particularly in children. Pyelocaliectasis is seen not 
only in obstruction but also in other conditions, such as residual dilatation after relief of obstruction, vesicoureteral reflux and pyelonephritis. The standard excretory urography (IVU), even with diuretic augmentation, does not permit one to diagnose or exclude objectively the presence of urinary obstruction. The Whitaker test (Whitaker, 1973) is considered by some authors to be the gold standard for the diagnosis of obstructive pyelocaliectasis but it is invasive and, therefore, has not gained wide use. Moreover, the intrinsic urine output of the kidney contributes an unknown volume to the total amount of fluid being infused particularly in children and the potential for false positive studies should be considered whenever the urine output of the corresponding kidney is high. Diuretic renography is the most widely accepted non-invasive procedure (Kass et al., 1985). However, it has the disadvantages of being expensive, using ionizing radiation and having a 10 to $15 \%$ rate of false-positive and indeterminate results (HowmanGiles et al., 1987).

Platt et al. (1989a) recently described the use of resistive index (RI) obtained during Doppler ultrasonography to differentiate obstructive from non-obstructive pyelocaliectasis. An RI of 0.70 has been emerged as the dividing line between obstructive and nonobstructive dilatation in adult population. However, the use of RI for diagnosis of obstructive uropathy has several limitations. It has recently been shown that the $\mathrm{Rl}$ is age dependent and frequently elevated above 0.70 in young children (Gilbert et al., 1993; Wong et al., 1989; Dejter et al., 1988; Keller, 1989). Moreover, certain types of renal medical diseases, such as acute tubular necrosis, interstitial nephritis and hemolytic uremic syndrome may cause elevations of RI (Platt et al., 1991). Therefore, predicting obstruction based upon a threshold level of 0.70 may be inaccurate particularly in children.

We have recently established a standard and stable model of unilateral partial ureteral obstruction in dogs (Shokeir, 1995). In the present study, we have used this model to evaluate the effect of different grades of partial obstruction upon the RI. The RI was assessed before and after the addition of intravenous normal saline and furosemide to determine additional changes in the RI that may be useful in the development of a pharmacologically based Doppler utlrasonographic method of evaluation of pyelocaliectasis.

\section{Materials And Methods}

Ten male mongrel dogs weighing $18-25 \mathrm{~kg}$. were used. The procedures were carried out under general anesthesia using thiopental 
sodium (10 mg./kg.) with endotracheal intubation and mechanical ventilation. Right partial ureteral obstruction was created as we previously described (Shokeir, 1995). A 6F ureteral catheter was inserted into the right ureteral orifice and was cut $2 \mathrm{~cm}$ distal to the orifice and fixed into the bladder mucosa. The most distal part of the ureter was ligated around the catheter. This model enables variation of the degree of obstruction by insertion of stylets of different diameters inside the lumen of the ureteric catheter. The dogs were stratified into 2 equal groups (5 dogs each) according to the degree of partial ureteral obstruction. Grade 1 obstruction was applied to group A while dogs of group B were subjected to grade 3 obstruction (Shokeir, 1995). Induction of partial ureteral obstruction was covered by antibiotic prophylaxis (amoxicilin $500 \mathrm{mg}$. twice daily for 3 days). Evaluations by Doppler ultrasonography were carried out before induction of ureteral obstruction and by the end of the eighth week of obstruction after stabilization of the configuration and function of both renal units. Bmode ultrasonography showed the different degrees of obstruction of the right kidney and absence of abnormalities of the left kidney.

Every animal was subjected to Doppler ultrasonography 3 times in one setting: 1) under control condition, before infusion of normal saline, 2) 30-60 minutes after intravenous infusion of normal saline (15 $\mathrm{ml} . / \mathrm{kg}$.) given in a rate of $1 \mathrm{ml} / \mathrm{kg} . / \mathrm{min}$., and 3) 10 minutes after administration of furosemide ( $1 \mathrm{mg} . / \mathrm{kg}$.). A urethral catheter was inserted throughout the entire study to measure urine output and replace it by intravenous saline.

\section{Technique of Doppler ultrasonography}

Ultrasound examinations were performed on a Toshiba (SSA270A) unit (Toshiba Corporation Medical Systems Division, Tokyo, Japan) using transducer frequency of $3.75 \mathrm{MHz}$. Renal morphology was studied in longitudinal and transverse planes. At least 5 Doppler spectra were obtained from more than 3 regions in each kidney in every study. Renal pulsatility indexes (PI) were obtained using Toshiba software from the formula: $\mathrm{PI}=$ (peak systolic velocity-end diastolic velocity) / mean velocity. The renal resistive indexes $(\mathrm{RI})$ were calculated as: $\mathrm{RI}=$ (peak systolic velocity-end diastolic velocity) / peak systolic velocity. Values of PI and RI used in statistical analyses were averages of those obtained at each time in each kidney.

Statistical analyses.

Statistical analyses were performed with an independent $t$ test and simple linear regression analysis. 


\section{Results}

All animals survived the experiments and no complications were observed. Satisfactory Doppler examinations were obtained in all 20 renal units. Representative waveforms at the different conditions of Doppler examinations are given in Figure 1. Combining all examinations, there was excellent positive correlation between PI and RI (correlation coefficient $=0.97, \mathrm{p}=0.0001$ ). Because of this positive correlation between the PI and Rl, we only discussed the changes of vascular resistance in terms of $\mathrm{RI}$.
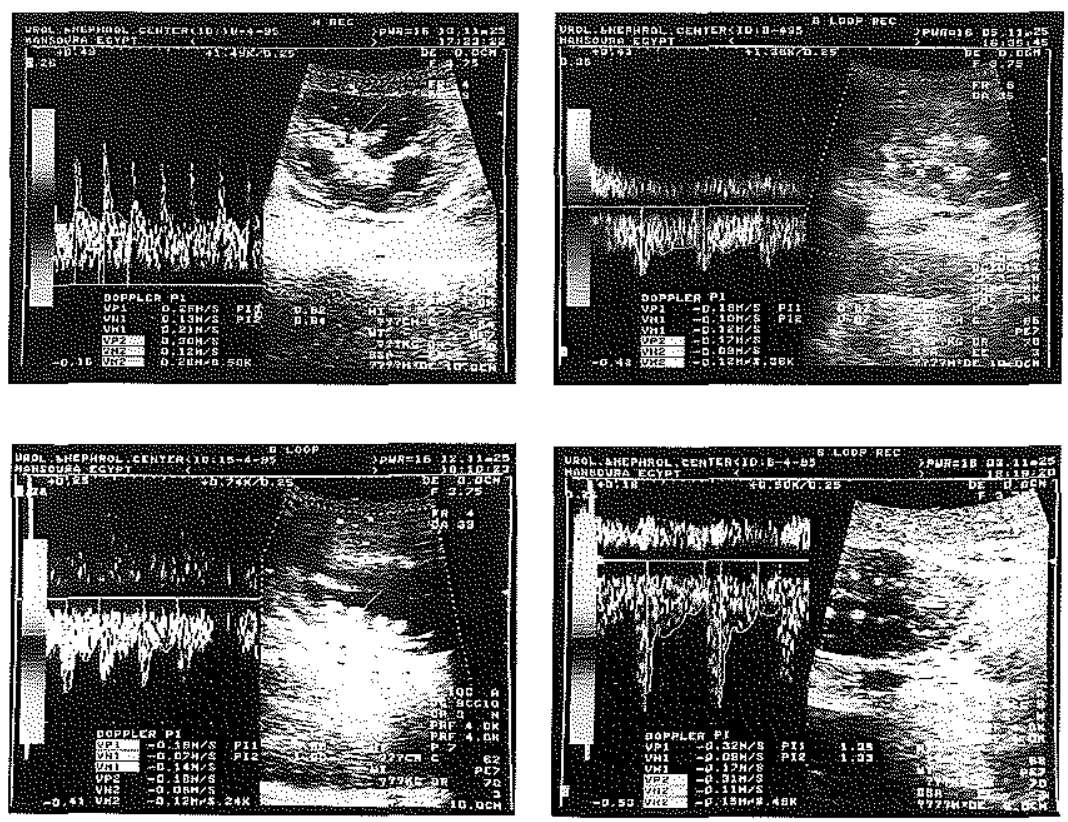

Figure 1: Representative Doppler waveforms of right kidney of group $A$ at different conditions of examination :a) preoperative (mean $\mathrm{Rl}=0.44$ ). b) after induction of right ureteral obstruction and infusion of saline (mean $\mathrm{Rl}=0.55$ ), c) after infusion of saline (mean $\mathrm{RI}=0.65$ ), and $\mathrm{d}$ ) after injection of firosemide (mean $\mathrm{RI}=0.69$ ).

\section{RI of the obstructed kidney}

For group A, the mean preoperative RI of the right kidney was $0.44 \pm 0.04$. After induction of mild degree of partial ureteral obstruction, the RI increased to $0.59 \pm 0.03(p<0.0001)$. Further increase to $0.65 \pm 0.04$ and $0.70 \pm 0.03$ were obtained for the RI of the right 
kidney 30-60 minutes after intravenous infusion of normal saline and 10 minutes after administration of furosemide, respectively. Significant increase could be observed if we compared the RI before and after saline infusion $(p<0.02)$. Moreover, comparison of RI after saline infusion with that after furosemide injection revealed a difference of statistical significance $(p<0.02)$ (Figure 2$)$.

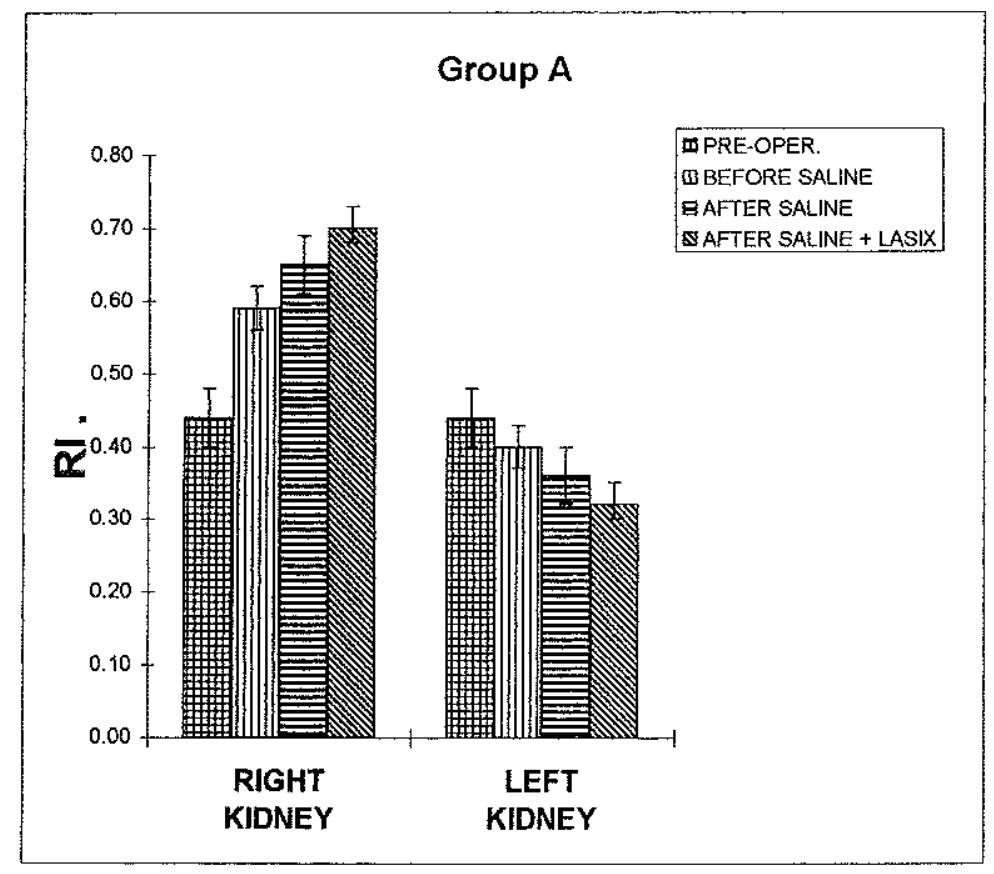

Figure 2 : Mean and standard deviation values of RI of both renal units in group A.

For group B, the mean preoperative RI of the right kidney was $0.46 \pm 0.06$. Induction of a severe degree of partial ureteral obstruction significantly increased the RI to $0.69 \pm 0.02(p<0.00001)$. Intravenous infusion of normal saline caused a further increase to $0.73 \pm 0.02$ and injection of furosemide further increased the RI to $0.79 \pm 0.02$. The difference between the mean values of RI before and after infusion of normal saline; and before and after administration of furosemide are statistically significant ( $p<0.001$ in each condition) (Figure 3 ). 


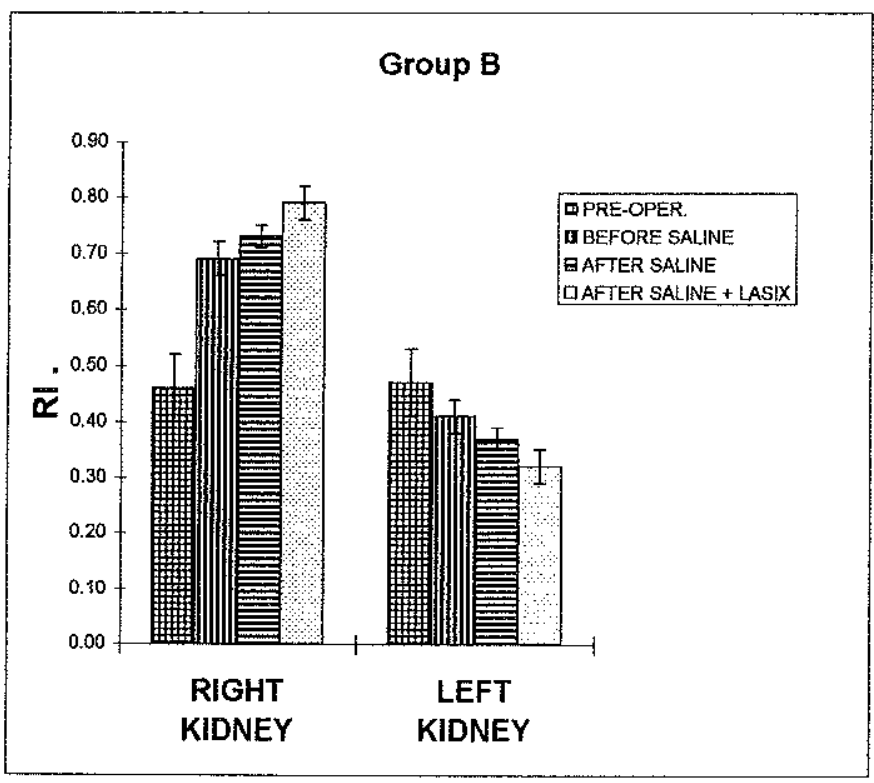

Figure 3: Mean and standard deviation values of RI of both renal units in group B.

RI of the non-obstructed kidney.

The mean preoperative values of $\mathrm{Rl}$ of the left kidney were 0.44 \pm 0.04 and $0.47 \pm 0.06$ for groups A \& B, respectively. After 8 weeks of obstruction of the right kidney, the mean RI of the left kidney significantly decreased to $0.40 \pm 0.03$ for group $A$, and $0.41 \pm 0.04$ for group $B,(p<0.05$ for both groups). After infusion of normal saline, RI values decreased to $0.36 \pm 0.03 \& 0.37 \pm 0.03$ for groups $A$ and $B$, respectively. After administration of furosemide a further decrease of RI was observed and values of $0.32 \pm 0.02 \& 0.32 \pm 0.02$ were reported for groups A and B, respectively. For both groups, the decrease of RI values after intravenous infusion of normal saline and after administration of furosemide is statistically significant $(p<0.05)$ (Figures 2,3 ).

\section{Right/Left RI ratio}

The ratio of $\mathrm{Rl}$ of the obstructed right kidney to its normal contralateral counterpart was calculated in both study groups and given in Figure 4. The mean ratio at preoperative condition was $1.02 \pm 0.04$ for group A and $0.98 \pm 0.15$ for group B. After induction of right partial ureteral obstruction the ratio significantly increased to $1.44 \pm 0.23$ for group $A$ and $1.69 \pm 0.13$ for group B. Infusion of normal saline also significantly increased the ratio to $1.80 \pm 0.22$ for group $\mathrm{A}$ and $2.00 \pm$ 
0.20 for group B. Furthermore, administration of furosemide caused a further significant increase of the ratio to $2.17 \pm 0.09$ and $2.44 \pm 0.19$ for groups $A$ and $B$, respectively. However, there was no significant difference of RI ratio between the 2 study groups at the different conditions of Doppler studies.

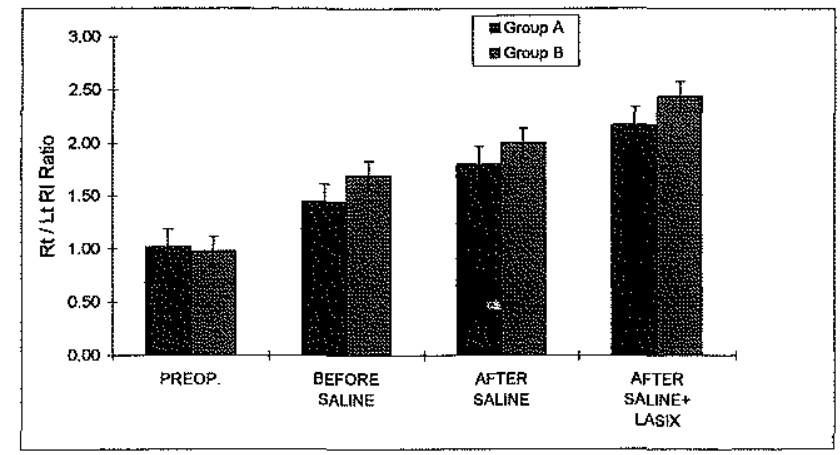

Figure 4: Right/left RI ratio in both study groups

\section{Discussion}

Mechanisms of renal failure in partial ureteral obstruction may be different from those in acute complete obstruction. Apart from accidental ligation or ureteric calculus, obstruction in humans is nearly always chronic and partial in nature. Because of this, we have endeavored to study renal vascular resistance in terms of Doppler waveforms in partial ureteral obstruction. We have used a standard model of stable partial ureteral obstruction which stimulates to a large extent, chronic and partial ureteral obstruction in humans (Shokeir, 1995). However, the duration of obstruction used in this experiment may not be fitting for pediatric obstruction which is usually developmental and stays for prolonged periods.

In 1989, Platt and associates demonstrated that an RI of 0.70 is the dividing line between obstructive and non-obstructive pyelocaliectasis in adults. However, it has been shown that several factors may affect the RI values such as age of the patient (Gilbert et al., 1993; Wong et al., 1989; Dejter et al., 1988; Keller, 1989), presence of certain medical renal diseases, (Platt et al., 1991), oral hydration (Palmer et al,, 1991), and the combination of intravenous infusion of normal saline and furosemide (Bude et al., 1994). Therefore, criteria predicting obstruction based upon a threshold level of 0.70 may be meaningless particularly in young children. In an effort to circumvent these shortcomings, Keller et al. (1991) advocated using a ratio of the Rl of the potentially obstructed 
kidney to its normal contralateral counterpart. Additionally, Palmer et al. (1991) and Keller et al. (1992), recently investigated Doppler ultrasonography in children before and after intravenous furosemide, showing that furosemide causes the RI to increase above baseline in normal and non-obstructive pyelocaliectatic kidneys. Renowden and Cochlin (1992) likewise have shown in adults that furosemide does not affect the Rl of normal kidneys and causes the index to increase above baseline in obstructed kidneys. In this series, we have shown that the combination of normal saline and furosemide can increase the index of obstructed kidneys and decrease the index of non-obstructed ones. Such a difference in response of non-obstructed kidneys could be attributed to the addition of normal saline in our series.

The present studies demonstrate that the RI of the nonobstructed normal kidney decreases after induction of obstruction of its contralateral counterpart. This could be explained by the reduction of renal vascular resistance associated with the compensatory hemodynamic and structural adaptations of the non-obstructed kidney (Restaino et al., 1990) or simply could be due to technical errors of the Doppler ultrasonography. Studies of larger number of renal units will help to solve this issue. Our observation that intravenous normal saline and furosemide can decrease the RI in non-obstructed kidneys is potentiated by the physiological fact that both normal saline and furosemide can decrease renal vascular resistance( Gerber and Nies, 1980; Ludens et al., 1968). Ludens and associates (1968) demonstrated a significant lowering of the renal vascular resistance in dogs after intravenous infusion of normal saline and furosemide. This decrease in renal vascular resistance was dose related. When the normal saline dosage was held constant, larger doses of furosemide decreased the vascular resistance. Similarly, with constant dose of furosemide, increased doses of saline decreased the vascular resistance. It is likely that a similar decrease in renal vascular resistance is reflected in the decrease of renal RI in our series.

Bude and associates (1994) have addressed the idea of a test that would identify obstruction, not based upon a single basal RI value, but upon a change in Rl through a standardized pharmacological test. For this test, they suggested the use of intravenous normal saline and furosemide. In their recent study, they have demonstrated that the combination of intravenous normal saline and furosemide can cause significant decrease of RI in 13 non-obstructed renal units. However, they could not certainly know whether this decrease is due to saline only or due to saline and furosemide. Moreover, their series does not include obstructed kidneys.

We have demonstrated that each of normal saline and furosemide has a significant role in changing the RI values in obstructed as well as in normal kidneys. Intravenous infusion of normal saline and 
furosemide causes the RI to increase in obstructed and to decrease in non-obstructed kidneys. These changes are reflected as a significant increase of the RI ratio between the obstructed and the normal kidneys. From a clinical perspective, the use of RI ratio will greatly enhance the ability of Doppler ultrasonography to distinguish between obstructive and non-obstructive pyelocaliectasis. The RI ratio is better than using a single RI value particularly in young children and certain types of renal medical diseases in which the RI is already elevated. Moreover, the RI ratio eliminates the factors that may affect the renal vascular resistance, rather than obstruction, such as renal colic and pyelonephritis (Urlich et al., 1995). However, this method is inapplicable to solitary kidneys or to situations in which both kidneys are abnormal or potentially obstructed. 

Chapter 4

Partial ureteric obstruction: a study of Doppler ultrasonography and diuretic renography in different grades and durations of obstruction

Ahmed A. Shokeir, Rien J.M. Nijman, Mohamed El-Azab and Abraham P. Provoost

Urology \& Nephrology Center, Mansoura University, Mansoura, Egypt; and Departments of Pediatric Urology and Pediatric Surgery, Erasmus University, Rotterdam, The Netherlands

Br. J. Urol. (1996) 78: 829-835 



\section{Abstract}

\section{Objectives}

To study the changes in renal resistive index (RI) and renal function with time during different grades of partial unilateral ureteric obstructions, and to determine the correlation between the ultrasonographic and renographic findings.

\section{Methods}

Ten dogs underwent right partial ureteric obstruction; grade 1 (mild) obstruction was applied in five dogs (group A) and grade 3 (moderate and severe) obstruction in the other five (group B). All dogs were assessed using excretory urography, diuretic renography with the calculation of half-time drainage $T_{1 / 2}$ and bilateral renal Doppler ultrasonography before the experiment began, after one week of obstruction, and every 2 weeks during 8 weeks of obstruction.

Results

In both groups, after induction of right ureteric obstruction, there was a progressive decrease of effective renal plasma flow (ERPF) and progressive increase of the RI of the right kidney at the end of the first and second weeks of obstruction, with an almost stable value thereafter. The decrease of ERPF and the increase of RI in the right kidney were correlated with the degree of obstruction. There was also a dramatic increase of $\mathrm{T}_{1 / 2}$ of the right kidney that correlated with the degree of obstruction. Concomitantly, there was a significant compensatory increase of ERPF and a significant decrease of the RI of the left kidney in both groups. The compensatory increase in ERPF limited the loss in total ERPF in both groups. The contribution of obstructed kidney to the total ERPF was significantly reduced in both groups. At the end of the eighth week, taking all kidneys together, there was a statistically significant negative correlation between the ERFP and RI, and between ERPF and $T_{1 / 2}$, and a positive correlation between $T_{1 / 2}$ and $R I$.

\section{Conclusions}

Unilateral partial ureteric obstruction increased the $R I$ and $T_{1 / 2}$ and decreased the ERPF of the corresponding kidney, together with a decrease of RI and an increase in ERPF of the contralateral kidney. The more severe the obstruction, the greater the increase in $\mathrm{RI}$ and $\mathrm{T}_{\mathrm{I} / 2}$ and the decrease in ERPF. After the obstruction stabilized $R I$ and $T_{1 / 2}$ were positively correlated. 


\section{Introduction}

There have been numerous animal studies over the past 20 years investigating the haemodynamic changes that occur with acute chronic ureteric obstruction (Ryan et al., 1987; Vaughan et al., 1970). Most of these studies have shown a definite rise in renal arterial vascular resistance when obstruction is present. However, earlier investigators used invasive and cumbersome methods to measure direct renal blood flow and to estimate renal vascular resistance. In 1989, Platt et al. hypothesized that renal resistance would produce a change in the wave characteristics of Doppler ultrasonography and showed that the renal resistive index (RI) was high in obstructed kidney. Since then, others have also suggested that high-grade renal obstruction is reflected in increased RI (Gilbert et al., 1993; Keller et al., 1989; Platt et al., 1991). To our knowledge, the changes in Doppler waveforms assessed for different durations and degrees of partial ureteric obstruction have not yet been fully investigated. Thus, we have examined the natural progression of Doppler waveforms recorded in various durations and for different grades of partial ureteric obstruction.

O'Reilly et al. (1978) and Koff et al. (1980) described characteristic patterns of the diuretic washout curve in normal, dilated unobstructed and obstructed upper urinary systems, with no attempt to quantify the rate in response to the diuretic. Unfortunately, many dilated systems had an intermediate response to the diuretic, making interpretation of the diuretic renogram alone subjective. In 1985, Kass et al. used half-time drainage $\left(\mathrm{T}_{1 / 2}\right)$ as an objective tool in the diagnosis of obstruction. $T_{1 / 2}$ is defined as the interval necessary for half of the radioisotope in the renal collecting system to be eliminated after the administration of frusemide. Kidneys with a $T_{1 / 2}<10 \mathrm{~min}$ are unobstructed, those with $T_{1 / 2}>20 \mathrm{~min}$ obstructed and those with a $T_{1 / 2}$ of $10-20 \mathrm{~min}$ intermediate (Dejter et al., 1988).

Doppler ultrasonography has recently been reported to differentiate obstructive from non-obstructive pyelocalyectasis. An RI of 0.70 is accepted by most investigators as the threshold between the obstructed and non-obstructed dilated upper urinary tract. Because the measurement of renal Rl is non-invasive, it has considerable appeal in its potential application of patients with obstructive uropathy. We determined whether the renal RI could be used to assess the degree of obstruction by comparing changes in $\mathrm{Rl}$ with changes in $T_{1 / 2}$ in a canine model of unilateral partial ureteric obstruction. 


\section{Materials and methods}

The study comprised 10 male mongrel dogs ( body weight 18$25 \mathrm{~kg}$ ). Operative procedures were carried out with the animals anaesthetized using thiopental sodium $(10 \mathrm{ml} / \mathrm{kg})$ with endotracheal intubation and mechanical ventilation. The study was approved by the local ethical committee.

All dogs underwent IVU, diuretic renography using ${ }^{99 \mathrm{~m}} \mathrm{Tc}-$ $\mathrm{MAG}_{3}$ and bilateral renal Doppler ultrasonography. The latter procedure was used to study the renal anatomy (B-mode imaging) and to measure the velocity of renal blood flow (Doppler scan), thus providing not only an anatomical but a functional assessment.

Partial ureteric obstruction was created as described previously (Shokeir, 1995). A $6 \mathrm{~F}$ ureteric catheter was inserted into the right ureteric orifice and was cut $2 \mathrm{~cm}$ distal to the orifice and fixed into the bladder mucosa. The most distal part of the ureter was ligated around the catheter. This model allows the degree of obstruction to be varied by inserting stylets of different diameters inside the lumen of the ureteric catheter. The dogs were stratified into two groups of five dogs each according to the degree of partial ureteric obstruction. Grade 1 obstruction was applied to group A and grade 3 to those in group B (Shokeir, 1995). The induction of partial ureteric obstruction was covered by antibiotic prophylaxis (amoxicilin $500 \mathrm{mg}$ twice daily for 3 days).

After surgery, all 10 dogs underwent were assessed by IVU,

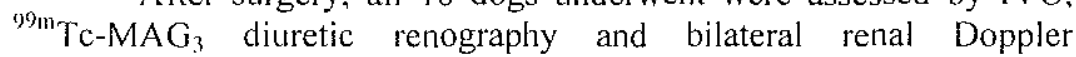
ultrasonography by the end of the first week and every 2 weeks for 8 weeks thereafter.

\section{Doppler ultrasonography}

The dogs were given intravenous normal saline $(15 \mathrm{~mL} / \mathrm{kg})$ before Doppler studies and a urethral catheter was inserted. The infusion of normal saline was continued during the entire Doppler studies to replace the urine output. Ultrasonography was performed using a Toshiba SSA-270A unit (Toshiba Corp, Japan) at a transducer frequency of $3.75 \mathrm{MHz}$, and the renal morphology was studied in longitudinal and transverse planes. At least five Doppler spectra were obtained from more than three regions in each kidney in every study. Renal pulsatility index (PI) was obtained using the Toshiba software from the formula: $\mathrm{PI}=$ (Peak systolic velocity-end diastolic velocity) / mean velocity. The renal resistive index (RI) was calculated as: $\mathrm{RI}=$ (peak systolic velocity-end diastolic velocity)/ peak systolic velocity. Values of PI and RI used in statistical analyses were means of those obtained at each time in each kidney. 


\section{Diuretic renography}

Diuretic renography was performed according to a standard protocol for all dogs. A urethral catheter was placed, an intravenous line was established and normal saline was given $(15 \mathrm{~mL} / \mathrm{kg})$. The dog was placed supine on the scanning table, $2.1 \mathrm{MBq} / \mathrm{kg}{ }^{90 \mathrm{~m}} \mathrm{Tc}-\mathrm{MAG}_{3}$ was injected intravenously and a standard renal scan was taken. After 20 $\mathrm{min}, 1 \mathrm{mg} / \mathrm{kg}$ frusemide was injected intravenously and sequential images acquired a computerized gamma camera system for $30 \mathrm{~min}$ and the computed data processed to generate a time-activity curve and to calculate the effective renal plasma flow (ERPF) and the half-time drainage $\left(\mathrm{T}_{1 / 2}\right)$ as described previously (Shokeir et al., 1994). Statistical analyses were performed using an independent or paired $t$-test and linear regression analysis.

\section{Results}

All animals survived the surgery and there were no complications. After the induction of partial ureteric obstruction, IVU and B-mode ultrasonography showed progressive hydro-ureteronephrosis that was increased in severity with the increasing grade and duration of obstruction (Shokeir, 1995). For group A, the renal morphology stabilized by the end of the second week in three dogs and by the end of the fourth week in the remaining two; the hydronephrosis was assessed as mild. In group B the hydronephrosis occurred more quickly and more severe, the renal morphology stabilizing by the end of fourth week in three dogs and by the end of the sixth week in two; the hydronephrosis was assessed as moderate and severe.

\section{Doppler ultrasonography of the right kidney}

Satisfactory Doppler examinations were obtained in all 20 renal units. There was a close positive correlation between PI and RI at all times of examinations $(r=0.87, P<0.001)$; because $P I$ and $R I$ were positively correlated, the changes in vascular resistance are discussed in terms of RI.

For group A, the mean (SD) initial RI of the right kidney was $0.44(0.04)$. By the end of the first week, the mean (SD) RI was unchanged at $0.44(0.07)(P>0.05)$. By the end of the second week, the RI significantly increased to $0.065(0.03)$, remaining stable thereafter (Fig. 1). Representative Doppler waveforms of the right kidney of one of the dogs of group $A$ at different durations of obstruction are given in Figure 2. For group $B$, the mean initial $\mathrm{Rl}$ of the right kidney was $0.46(0.06)$ and the RI increased significantly to $0.68(0.03)$ at the end of 
the first week and $0.72(0.01)$ at the end of the second week; thereafter, RI remained stable (Figure I). Representative Doppler waveforms of the right kidney of one of dogs in group $B$ at different durations of obstruction are given in Figure 3. The mean values of Rl of group B were significantly higher than those of group $A$ at all times of obstruction (Figure 1).

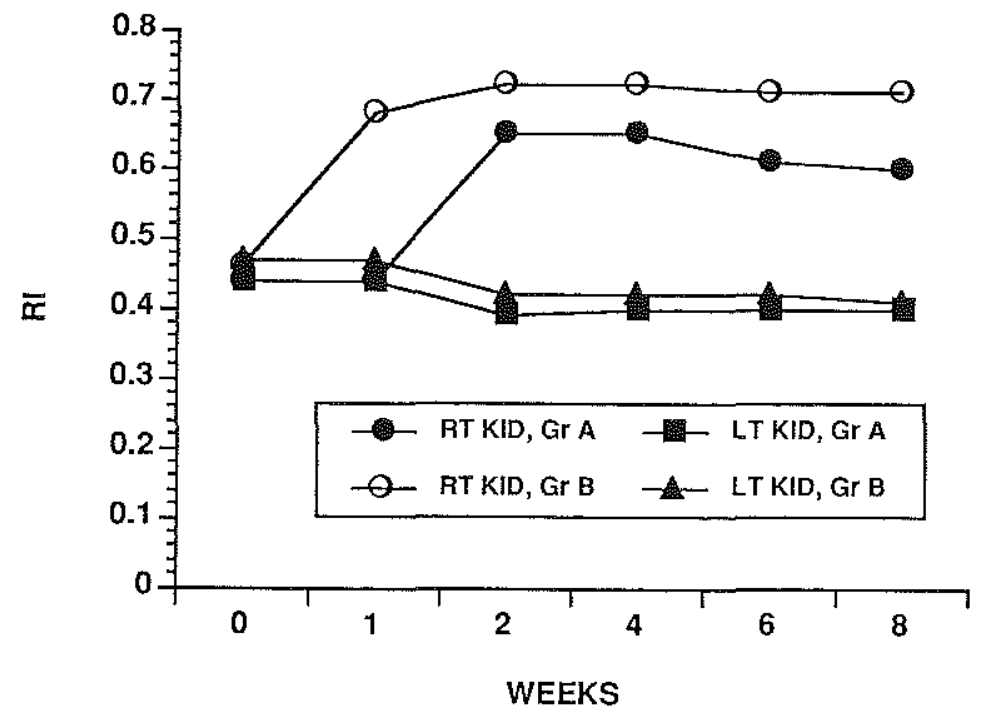

Fig. I : The initial Rl of both kidneys and at different times during right ureteric obstruction in both groups.

Doppler ultrasonography of the left kidney

Initially, the mean (SD) RI values of the left kidney were $0.44(0.04)$ and $0.47(0.06)$ for groups $\mathrm{A}$ and $\mathrm{B}$, respectively. By the end of the eighth week, the RI was significantly decreased to $0.40(0.03)$ and $0.41(0.04)$ for groups $\mathrm{A}$ and $\mathrm{B}$, respectively (Figure 1). There were no significant difference between the mean RI of groups A and B at any time.

\section{Changes in ERPF of the right and left kidneys}

Figure 4 shows the changes in ERPF of the obstructed (right) and contralateral (left) kidneys after partial ureteric obstruction. For group A, the initial mean (SD) ERPF of the right kidney was $286(40)$ $\mathrm{mL} / \mathrm{min}$. After the induction of right partial ureteric obstruction the mean ERPF of the obstructed kidney decreased significantly to 230 (37) and $174(17) \mathrm{mL} / \mathrm{min}$. by the end of the first and second weeks, respectively, remaining stable thereafter. For group B, the initial mean 
(SD) ERPF of the right kidney was $287(36) \mathrm{mL} / \mathrm{min}$; there was a significant progressive reduction of ERPF, with mean values of 144(37) and $111(17) \mathrm{mL} / \mathrm{min}$ by the end of the first and second weeks of obstruction. After the fourth week of obstruction, the ERPF was 89(28) $\mathrm{mL} / \mathrm{min}$ and there was no further decrease in ERPF. At all times during obstruction, the mean values of ERPF of the right kidney of groups $B$ were significantly lower than those of group A.

Initially, the mean (SD) ERPF of the left kidney was 271(64) and $258(67) \mathrm{mL} / \mathrm{min}$ for groups $\mathrm{A}$ and $\mathrm{B}$, respectively. After the induction of right partial ureteric obstruction, there was a progressive compensatory increase in ERPF of the left kidney. By the end of the eighth week, the mean (SD) ERPF of the left kidney was 315(58) and $327(28) \mathrm{mL} / \mathrm{min}$, for groups $\mathrm{A}$ and $\mathrm{B}$, respectively, with the increase in both groups being statistically significant compared to basal levels. In both groups, the compensatory increase in ERPF was most marked during the first 4 weeks and remained almost stable thereafter. The degree of the compensatory increase of ERPF of the left kidney was not significantly different in the dogs of both groups.

Due to the compensatory increase in ERPF of the contralateral kidney, the loss in total ERPF during unilateral partial obstruction was limited. Total ERPF in groups A and B stabilized at about $85 \%$ and $75 \%$ of the basal level, respectively, and the difference was not significantly different.

\section{Changes in the contribution of the obstructed kidney to total ERPF}

Because the changes in ERPF of the obstructed and the contralateral kidneys were opposite, the contribution of the obstructed kidney to total ERPF decreased with time. In group A, the contribution of the obstructed kidney decreased from 52 to $35 \%$ of total; the decrease in group B was significantly more severe, from 53 to $19 \%$ of the total. 

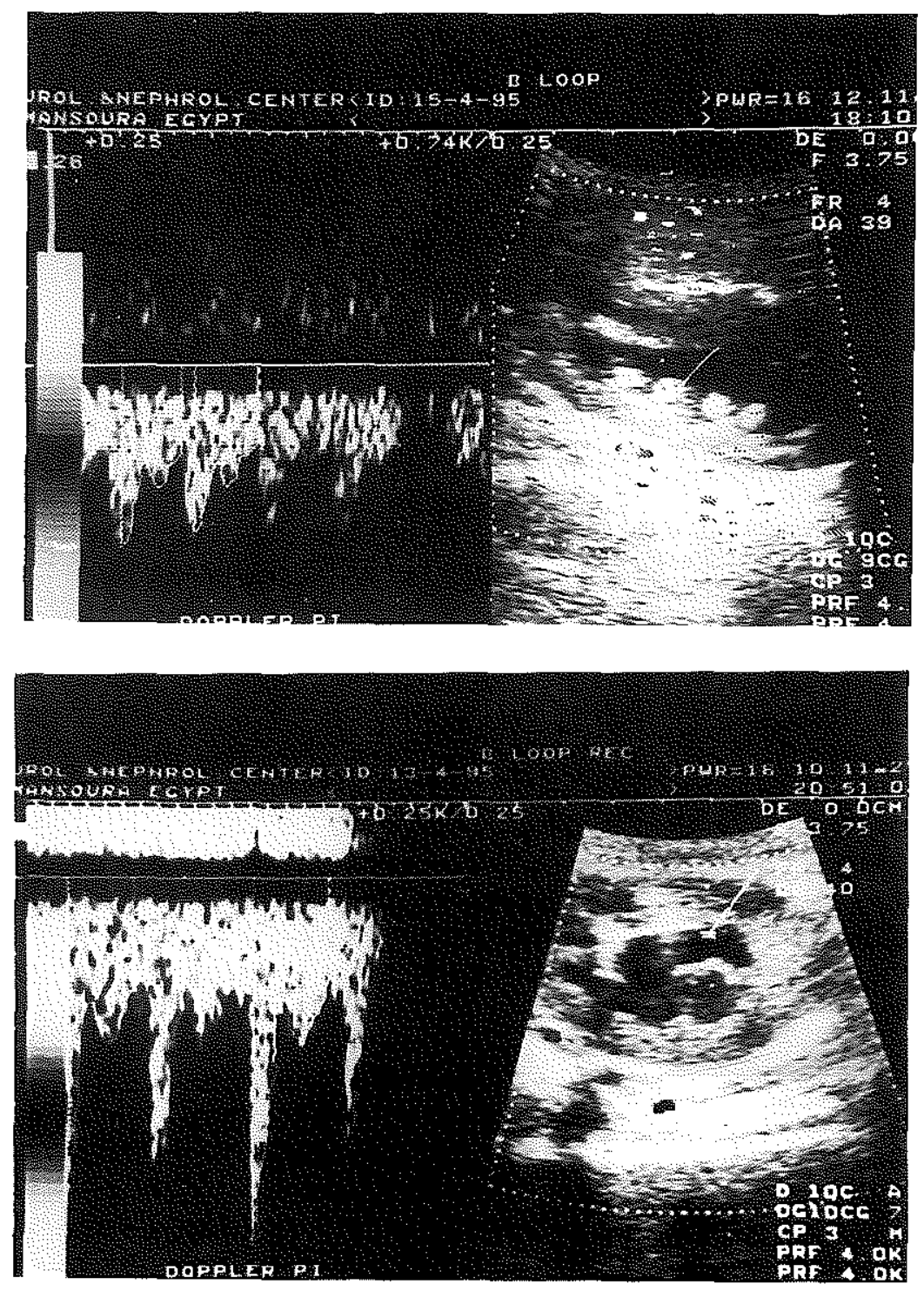

Fig. 2: Doppler ultrasonography of a right kidney of one of the dogs in group $\mathrm{A}$, a. at the end of the second week of obstruction (minimal liydronephrosis, mean $\mathrm{R} I=0.60$ ), and $\mathrm{b}$. by the end of the eighth week of obstruction (mild hydronephrosis, mean $\mathrm{Rl}=0.64$ ) 

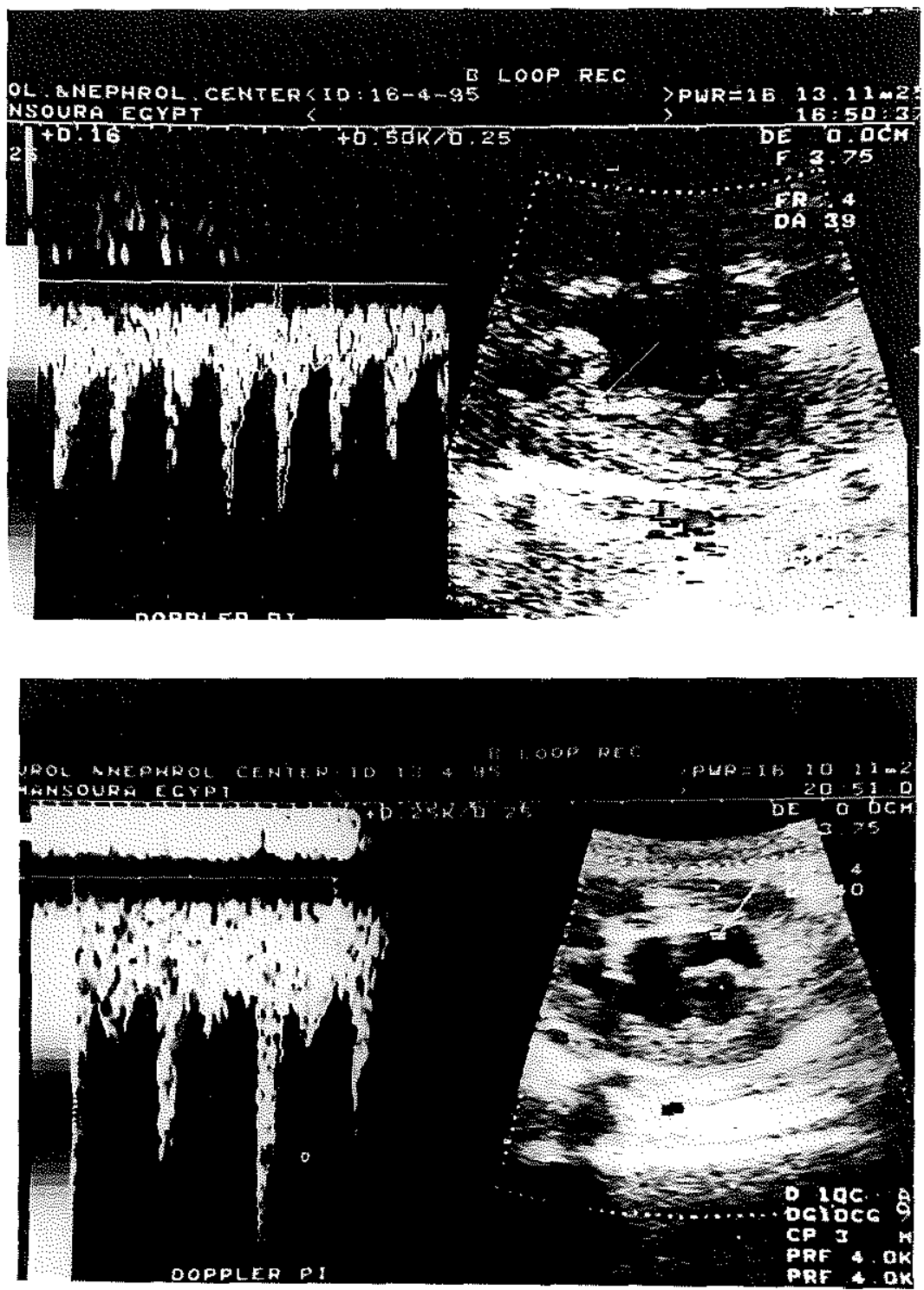

Figure 3: Doppler ultrasonography of a right kidney of one of the dogs in group B, a. by the end of the second week of obstruction (moderate lyydronephrosis, $\mathrm{Rl}=0.67$ ), and $\mathrm{b}$, by the end of the eighth week of obstruction (marked hydronephrosis, mean $R I=0.7$ I). 


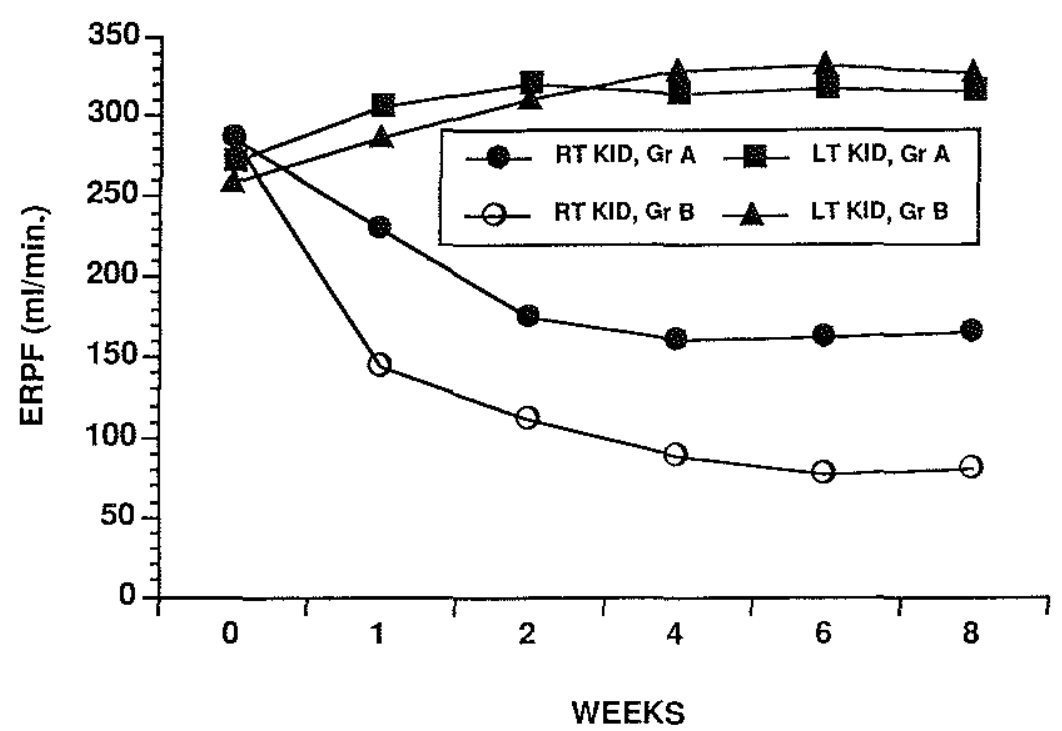

Figure 4: Progress of effective renal plasma flow (ERPF) of the corresponding (right) and contralateral (left) kidneys of both groups at all times during right ureteric obstruction.

Changes in $T_{1 / 2}$

Initially, the mean (SD) $\mathrm{T}_{1 / 2}$ of the right kidney was $4.6(2.3)$ and $4.9(2.4)$ min for groups $\mathrm{A}$ and $\mathrm{B}$, respectively. After the induction of right partial ureteric obstruction, there was a dramatic increase of $\mathrm{T}_{1 / 2}$ in both groups, with mean values of 14.4(1.8) and $31 \mathrm{~min}$ by the end of the first week for groups $\mathrm{A}$ and $\mathrm{B}$, respectively. The $\mathrm{T}_{1 / 2}$ values of the right kidney of group $B$ were significantly higher than those of group $A$ at all points of obstruction. In contrast, there was no significant change of $T_{1 / 2}$ of the left kidney in both groups after the induction of obstruction of the right kidney. The mean values of $T_{1 / 2}$ of both renal units in the two groups are given in Figure 5.

\section{Correlations between variables}

Before obstruction, there were no significant correlations between ERPF and RI, ERPF and $T_{1 / 2}$ and between $T_{1 / 2}$ and RI. During obstruction, a significant negative correlation developed between ERPF and RI. At the end of the eighth week, taking the kidneys of all 10 dogs together, there was a significant negative correlation between ERPF and RI $(\mathrm{r}=-0.942, \mathrm{p}<0.001)$, and between ERPF and $\mathrm{T}_{1 / 2}(\mathrm{r}=-0.924, \mathrm{p}<$ $0.005)$. Furthermore, there was a significant positive correlation between $\mathrm{T}_{1 / 2}$ and $\mathrm{RI}(\mathrm{r}=0.948, \mathrm{p}<0.001)$. 


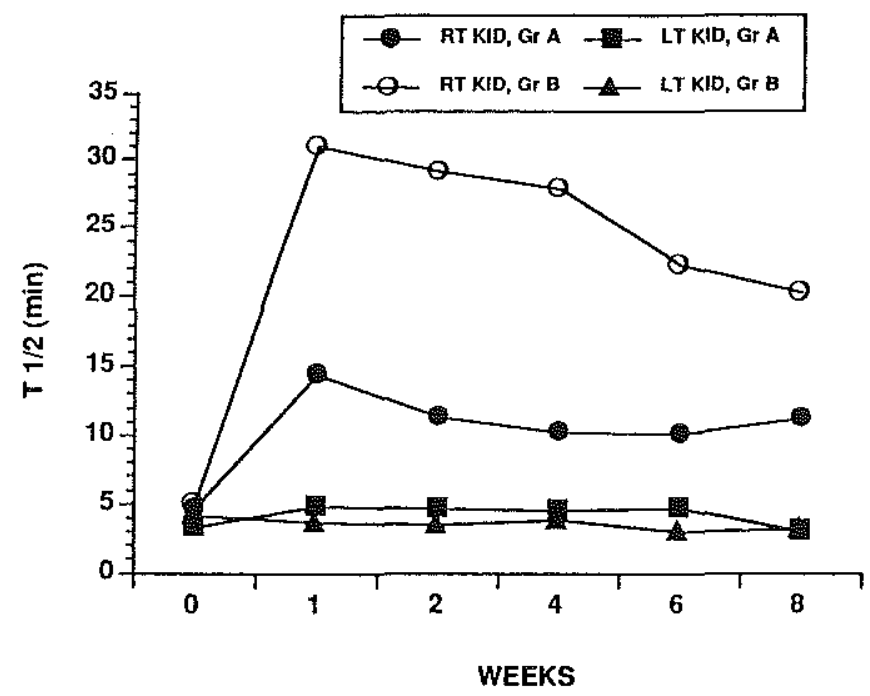

Figure 5 : Mean values of half-time drainage $\left(\mathrm{T}_{1 / 2}\right)$ of both renal units in the two groups of dogs with the duration of right ureteric obstruction.

\section{Discussion}

Mechanisms of renal failure in partial ureteric obstruction may be different from those in acute complete obstruction. Apart from accidental ligation or ureteric calculus, obstruction in humans is nearly always chronic and partial. Thus, we examined renal vascular resistance using Doppler ultrasonography in partial ureteric obstruction using a standard model of stable partial ureteric obstruction which largely simulates chronic and partial ureteric obstruction in humans (Shokeir, 1995).

The mean (SD) values of PI and RI in dogs, at $0.64(0.08)$ and $0.45(0.05)$, respectively, are much lower than those in humans, at 0.99 $(0.21)$ and 0.57 (0.06), respectively (Chen et al., 1993). In human obstructed kidneys, the threshold values of PI and RI are 1.50 and 0.70 (Chen et al., 1993), respectively, while in canine obstructed kidneys, the present threshold values were 0.90 and 0.60 , respectively.

Most investigators agreed that marked partial ureteric obstruction causes an increase of intra-renal vascular resistance that is reflected in an elevated PI and RI. The increase of vascular resistance in mild partial ureteric obstruction is still a matter of controversy. While this concept has been supported by some (Ryan et al., 1987; Restaino et 
al., 1990), it has been questioned by others (Chevalier et al., 1986). Different results could be explained by different definition of mild obstruction; the present study helps to resolve this issue, for there was a significant increase of RI at 2 weeks after the induction of mild partial ureteric obstruction in a standard and stable experimental model.

Chen et al. (1993), reported that in partial unilateral obstruction, the RI of the contralateral kidney is unchanged. Naturally, in human studies the RI of the contralateral kidney could not be known before occurrence of obstruction of the other kidney. After obstruction of one kidney, the RI of the contralateral kidney is always compared to that of a normal control group. The change in RI of the contralateral kidney may be so small that it could not be detected except in a large series of patients. The experimental models are more sensitive, as the same kidney serves as a control.

That the RI of the contralateral normal kidney is decreased after obstruction of the other kidney may be explained by a decrease in renal vascular resistance of the contralateral kidney due to compensatory hypertrophy (Ichikawa et al., 1979). Whether compensatory hypertrophy produces a change in Doppler waveforms could be examined in humans by studying of a group of live-kidney donors before and after nephrectomy.

It has been estimated that an RI of 0.70 is the threshold distinguishing an obstructive and non-obstructive dilated upper urinary tracts. The accuracy of this discriminatory value of RI can be improved by evaluating the contralateral kidney, especially in acute obstruction in which the RI may not yet have exceeded 0.70. Platt et al. (1991) suggested that a difference of $\geq 0.10$ between the obstructed and the contralateral kidney potentiated the diagnosis of obstruction. In the present study, unilateral obstruction increased the RI of the corresponding kidney and decreased RI in the contralateral kidney. This divergent response may be more valuable in the diagnosis of obstruction than a single discriminatory value.

At present, the diuretic renogram is used at most centers as the gold standard for the diagnosis of obstruction. An added benefit to radioisotope renography is the ability to measure differential GFR, thus obtaining the percentage of the total GFR contributed by each kidney. The calculation of half-time draining is more objective in the diagnosis of obstruction; the present results show that RI correlated closely with $\mathrm{T}_{\mathrm{V} / 2}$ in severe and mild partial ureteric obstruction; thus RI is a useful modality in the evaluation of hydronephrosis, even in its early and mild phases. These results agree with those of Gilbert et al. (1993) where values from renal Doppler ultrasonography were compared using drainage values obtained with renal scintigraphic paediatric kidneys and were correlated closely. In contrast. Chen et al. (1993) stated that not all 
renal obstructions have significantly elevated values of RI; in cases of mild obstruction there may be low and normal RI values.

The renal Doppler examination provides physiological information and is insufficient if precise anatomical information is required (Gilbert et al., 1993). Therefore, it cannot be used as a single modality to evaluate a patient with hydronephrosis. Thus, the value of using RI is in providing corroboration of the initial diuretic renogram, and as a noninvasive modality for monitoring the dilated collecting system under observation. Hence, a hydronephrotic kidney with a nonobstructive $T_{1 / 2}$ on diuretic renography can be followed closely with renal Doppler ultrasonography, obviating the need for frequent isotopic renal scintigraphy. Additionally, this method provides a non-invasive modality for monitoring patients after re-constructive surgery of the upper urinary tract.

In the present study, changes in ERPF of the obstructed kidneys occurred within the first 2 weeks of obstruction and remained almost stable thereafter. This finding is in agreement with similar studies of partial ureteral obstruction with a normal contralateral kidney (Stecker and Gillenwater, 1971; Claesson et al., 1983). Stecker and Gillenwater (1971) found that the maximal depression in function occurred during the first week of partial unilateral obstruction and remained almost stable by the $16^{\text {th }}$ week. Similarly, Claesson et al. (1983) induced partial obstruction of the left ureter in rats and significant changes in configuration and function of the obstructed kidney developed early (during the first 2 weeks) and did not progress significantly after that.

In the present study, the compensatory increase of ERPF of the left kidney occurred during the first 4 weeks and remained stable thereafter. In similar experiments in rats, compensatory renal growth continued rapidly for 2-3 weeks, began to stabilize after 4-6 weeks and did not apparently change beyond that time (Mitchell et al., 1974).

We have shown that the rate and magnitude of the compensatory response to mild obstruction (group A) was approximately the same as the response to severe obstruction (group B). This confirms the speculation that the progression of compensatory renal growth is an all or nothing phenomenon. If the threshold amount of kidney tissue is damaged or removed, then compensatory renal growth begins and progresses until compensatory growth is completed (Mitchell et al., 1974). 
Partial ureteral obstruction: role of renal resistive index in stages of obstruction and release

Ahmed A. Shokeir, Rien J.M. Nijman, Mohamed El-Azab, And Abraham P. Provoost

Urology \& Nephrology Center, Mansoura University, Mansoura, Egypt and Departments of Pediatric Urology and Pediatric Surgery, Erasmus University, Rotterdam, The Netherlands

Urology (1997) 49: 528-535 



\begin{abstract}
Objectives

To study the changes in renal resistive index (RI) and renal function before and after release of different grades of partial unilateral ureteral obstructions.
\end{abstract}

\title{
Methods
}

Ten dogs were subjected to right partial ureteral obstruction. Grade I (mild) obstruction was applied to 5 dogs (group A) and grade 3 (moderate and severe) obstruction was applied to the other 5 dogs (group B). Obstruction was maintained for 8 weeks followed by release of obstruction. All dogs were subjected to excretory urography, techetium-99m mercaptoacetyltriglycine diuretic renography with calculation of half-time drainage $\left(\mathrm{T}_{/ / 2}\right)$ and bilateral renal Doppler ultrasonography before the start of the experiment, after 8 weeks of obstruction, and every 2 weeks during the 8 weeks after release of obstruction.

Results

In both groups, after induction of right ureteral obstruction, there was a dramatic decrease of effective renal plasma flow (ERPF), increase of $\mathrm{Rl}$ and increase of $\mathrm{T}_{1 / 2}$ of the right kidney. Relief of obstruction was associated with normalization of $T_{1 / 2}$, reversal of $R I_{\text {, }}$ and recovery of ERPF to near basal values. No correlation was found between ERPF at the end of the recovery period and the functional parameters $\left(T_{1 / 2}, \mathrm{Rl}\right.$, or ERPF) of the obstructed kidney before release of obstruction.

\section{Conclusions}

(1) Unilateral partial ureteral obstruction produces an elevation of RI and $T_{1 / 2}$ and a fall in ERPF of the corresponding kidney. (2) After relief of obstruction, renal function is regained with associated reversal of RI. (3) Functional parameters (T $T_{1 / 2}, \mathrm{RI}$, or ERPF) of the obstructed kidney do not predict the recovery of ERPF after release of obstruction. (4) Rapid reversal of a previously elevated RI is an early indicator of recoverability of renal function after relief of ureteral obstruction. 


\section{Introduction}

It is established that the physiologic hallmark of significant obstructive uropathy is a decrease in renal blood flow or an increase in renovascular resistance. Thus, a noninvasive test to identify an increase in renovascular resistance is of obvious clinical use in the differentiation of patients with anatomical hydronephrosis without physiologic significance from patients with true obstructive uropathy. In 1989, Platt et al. described the use of Doppler ultrasonography as a noninvasive test to differentiate obstructive from nonobstructive pyelocaliectasis. They demonstrated a high renal resistive index (Rl) in obstructed kidneys. Since then, others have also suggested that high-grade renal obstruction is reflected in elevated RI (Gilbert et al., 1993; Keller et al., 1989; Platt et al., 1991).

Using our own canine model of stable partial ureteral obstruction (Shokeir, 1995), we have recently demonstrated that RI is correlated to the degree of obstruction; the more severe the obstruction, the more the RI is elevated (Shokeir et al., 1996a). Moreover, in other experimental (Shokeir et al., 1997a) as well as clinical studies (Shokeir et al., 1996b; Shokeir et al., 1996c) we have shown that the addition of intravenous normal saline and furosemide causes the RI to increase in the obstructed kidney and to decrease in nonobstructed kidney.

The present article is a continuation of our previous experimental work on the role of renal RI in unilateral partial ureteral obstruction. The aim of this work is to investigate the changes in RI, half-time drainage $\left(T_{1 / 2}\right)$ and effective renal plasma flow (ERPF) before and after release of different grades of partial ureteral obstruction and to determine whether these changes could be useful in predicting recoverability of renal function before corrective surgery.

\section{Materials and methods}

\section{Experimental Animals}

Ten male mongrel dogs weighing 18 to $25 \mathrm{Kg}$ were used. The procedures were carried out under general anesthesia using thiopental sodium $(10 \mathrm{mg} / \mathrm{kg})$ with endotracheal intubation and mechanical ventilation.

\section{Preoperative Assessment}

All dogs were subjected to intravenous urography (IVU),

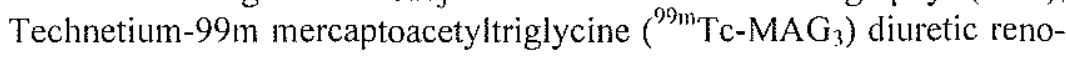


graphy and bilateral renal Doppler ultrasonography. The latter procedure was used to study the renal anatomy (B-mode imaging) and to measure the velocity of renal blood flow (Doppler scan), thus providing not only an anatomical assessment but a functional assessment as well.

\section{Operative Procedures and Experimental Groups}

Partial ureteral obstruction was created as we previously described (Shokeir, 1995). A 6F ureteral catheter was inserted into the right ureteral orifice and was cut $2 \mathrm{~cm}$ distal to the orifice and fixed into the bladder mucosa. The most distal part of the ureter was ligated around the catheter. This model enables variation of the degree of obstruction by insertion of stylets of different diameters inside the Iumen of the ureteric catheter. The dogs were stratified into 2 equal groups (5 dogs each) according to the degree of partial ureteral obstruction. Grade I obstruction was applied to group A, whereas dogs of group B were subjected to grade 3 obstruction. Induction of partial ureteral obstruction was covered by antibiotic prophylaxis (amoxicilin $500 \mathrm{mg}$ twice daily for 3 days).

The ureteral catheter used was semirigid plastic tube (Porges, 24200, Sarlat, France). Its external diameter was $1.8 \mathrm{~mm}$ and the intenal diameter was $0.96 \mathrm{~mm}$. Plastic stylets of two different sizes were left inside the lumen: for group A, a stylet with $0.40 \mathrm{~mm}$ diameter (stylet of $4 \mathrm{~F}$ ureteral catheter) and for group B, a stylet with $0.78 \mathrm{~mm}$ diameter (stylet of $6 \mathrm{~F}$ ureteral catheter).

By the end of eighth week of obstruction, the dog was reopened, the catheter was removed and ureterovesical reimplantation was carried out using our own technique of extravesical seromuscular tunnel (El-Hammady and Shokeir, 1994; Shokeir and El-Hammady, 1996).

\section{Postoperative Assessment}

All 10 dogs were subjected to IVU, ${ }^{9}{ }^{2} \mathrm{Tc}-\mathrm{MAG}_{3}$ diuretic renography, and bilateral renal Doppler ultrasonography by the end of the eighth week of obstruction, 1 week after release of obstruction, and then every 2 weeks for 8 weeks after release of obstruction. Ascending cystography was carried out by the end of the first week after release of obstruction.

\section{Technique of Doppler Ultrasonography}

The dogs were given intravenous normal saline $(1 \mathrm{~mL} / \mathrm{kg} / \mathrm{min}$ for 15 minutes) before Doppler studies and a urethral catheter was 
inserted. Infusion of normal saline to replace urine output was continued during the entire course of Doppler studies. Ultrasound examinations were performed on a Toshiba (SSA-270A) unit (Toshiba Corporation Medical Systems Division, Tokyo, Japan) using transducer frequency of $3.75 \mathrm{MHz}$. Renal morphology was studied in longitudinal and transverse planes. At least five Doppler spectra were obtained from more than three regions in each kidney in every study. Renal pulsatility indexes $(\mathrm{PI})$ were obtained using Toshiba software from the formula: PI = (peak systolic velocity-end diastolic velocity)/ mean velocity. The renal RIs were calculated as follows: $\mathrm{RI}=$ (peak systolic velocity-end diastolic velocity) / peak systolic velocity. Values of PI and RI used in statistical analyses were averages of those obtained at each time in each kidney.

\section{Technique of diuretic renography}

Diuretic renography was performed according to a standard protocol for all dogs. In each dog a urethral catheter was placed, an intravenous line was established and a normal saline was given $(1 \mathrm{~mL} / \mathrm{kg} / \mathrm{min}$ for 15 minutes). The dog was placed on the scanning table in the supine position and ${ }^{109} \mathrm{Tc}_{\mathrm{M}} \mathrm{MAG}_{3}$ was injected intravenously in a dose of $2.1 \mathrm{MBq} / \mathrm{kg}$, and a standard renal scan was performed. After 20 minutes, $1 \mathrm{mg} / \mathrm{kg}$ furosemide was injected intravenously. Sequential images were acquired into a gamma camera computer system for 30 minutes and the computed data then were processed for generation of a time-activity curve and calculation of ERPF and the $T_{1 / 2}$. Our technique of diuretic renography was previously described (Shokeir et al., 1994).

\section{Statistical analysis}

Statistical analyses were performed with an independent or paired $t$-test. Linear regression analysis was used to correlate the ERPF at the end of the recovery period with parameters of renal function obtained before release of obstruction.

\section{Results}

All animals survived the experiments and no complications were observed after induction of ureteral obstruction or after its release. IVU, gray-scale ultrasonography and ascending cystography

After induction of partial ureteral obstruction, IVU and B-mode ultrasonography demonstrated progressive hydroureteronephrosis that 
increased in severity with the increase of both the grade and duration of obstruction (Shokeir, 1995). For group A, the hydronephrosis was assessed as mild. In group B, the hydronephrosis was assessed as moderate and severe. After release of right ureteric obstruction, all the corresponding renal units regained a perfect configuration as shown by IVU (Fig. I A. B). Moreover, none of the 10 dogs showed vesicoureteral reflux by ascending cystography (Fig. 1-C).

\section{Doppler ultrasonography studies of the right kidney}

Satisfactory Doppler examinations were obtained in all 20 renal units. There was excellent positive correlation between PI and RI at all time points of examination (correlation coefficient $=0.87, p=0.0001$ ). Because the PI and Rl were positively correlated, we only discussed the changes of vascular resistance in terms of RI .

For group $\mathrm{A}$, the mean $\mathrm{RI}$ of the right kidney at basal condition was $0.44 \pm 0.04$. By the end of the eighth week of obstruction, the mean RI was $0.60 \pm 0.05$, indicating a significant increase $(p<0.0001)$. One week after release of obstruction, the $\mathrm{RI}$ almost returned to its basal condition, with a mean value of $0.46 \pm 0.04$ and remained stable thereafter (Fig. 2). Representative Doppler waveforms of the right kidney of one of group A dogs is given in Figure 3.

For group B, induction of right ureteric obstruction significantly increased the RI from $0.46 \pm 0.06$ at basal condition to $0.71 \pm 0.01$ by the end of the eighth week of obstruction $(p<0.00001)$. One week after relief of obstruction, the $\mathrm{R}$ I returned to its basal level with a mean value of $0.47 \pm 0.02$. The RI remained stable throughout the entire period of obstruction release (Figure 2). During the stage of obstruction, the mean $\mathrm{RI}$ values of group B were significantly higher than those of group A.

\section{Changes in ERPF of the right kidney}

For group A, the mean ERPF of the right kidney at basal condition was $286 \pm 40 \mathrm{~mL} / \mathrm{min}$. By the end of eighth week of obstruction, the ERPF significantly decreased to $164 \pm 16 \mathrm{~mL} / \mathrm{min}$. After release of obstruction, there was a progressive improvement of the ERPF starting by $192 \pm 21 \mathrm{~mL} / \mathrm{min}$ by the first week and attaining $248 \pm 28 \mathrm{~mL} / \mathrm{min}$ by the end of the eighth week (Fig. 4 ). 

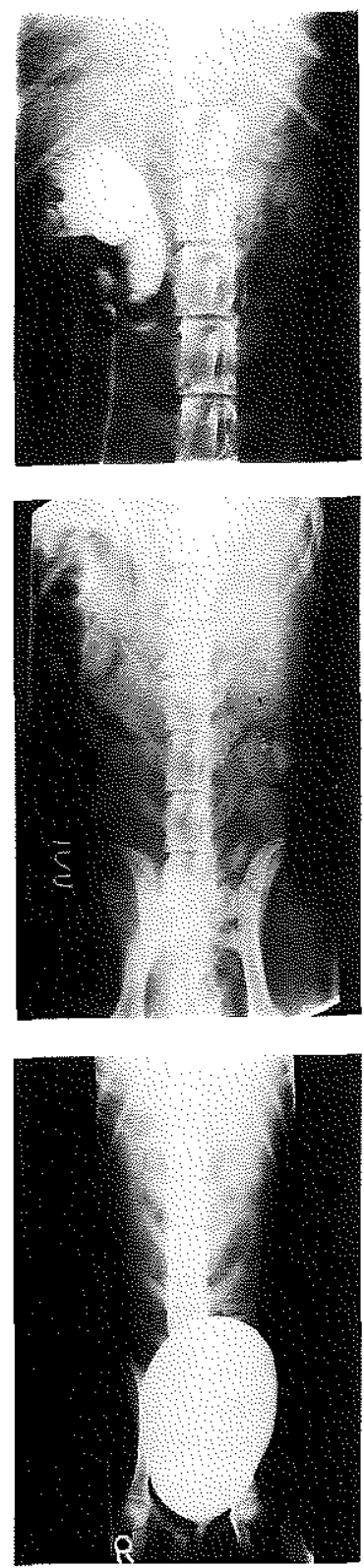

Fig. 1: Radiologic profile of one of the group B dogs: (A) IVU 8 weeks after right partial ureteral obstruction; (B) IVU 8 weeks after release of obstruction; and (C) ascending cystogram 1 week after release of obstruction and ureterovesical re-implantation. 


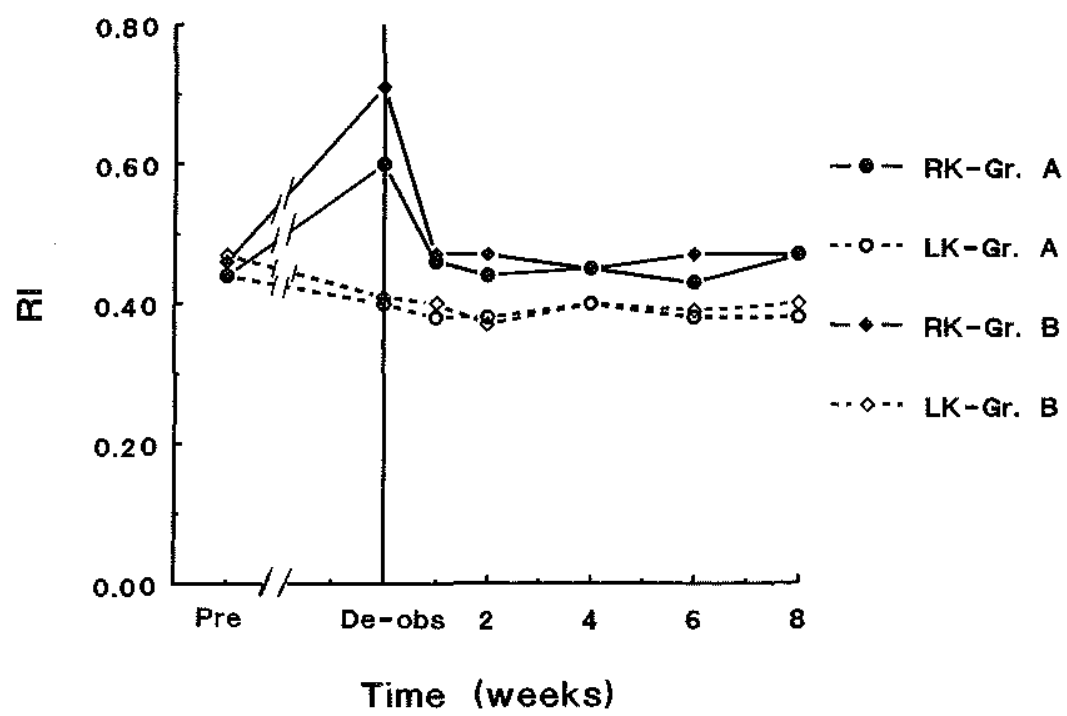

Fig. 2: Resistive index (RI) of both kidneys for the two study groups at basal condition, 8 weeks after right ureteral obstruction, and at different time points of release of obstruction (closed circle: right kidney, group $A$; open circle: left kidney, group $A$; closed, diamond, right kidney, group B; open diamond, left kidney, group B)..

For group $\mathrm{B}$, induction of right ureteric obstruction significantly decreased the ERPF from the basal level of $287 \pm 36 \mathrm{~mL} / \mathrm{min}$ to $80 \pm 42$ $\mathrm{mL} / \mathrm{min}$ at the eighth week of obstruction. After relief of obstruction, the right kidney started to regain its function and the ERPF increased from $113 \pm 18 \mathrm{~mL} / \mathrm{min}$ at the first week to $216 \pm 23 \mathrm{~mL} / \mathrm{min}$ at the eighth week after release of obstruction (Fig. 4).

\section{Changes in $T_{1 / 2}$ of Right Kidney}

At basal condition, the $T_{1 / 2}$ mean values of the right kidney were $4.6 \pm 2.3$ and $4.9 \pm 2.4$ minutes for groups $A$ and $B$, respectively. After induction of right partial ureteral obstruction, there was a dramatic increase of $T_{1 / 2}$ in both groups, with mean values of $11 \pm 1.8$ and $20 \pm$ 1.9 minutes by the end of the eighth week for groups $A$ and $B$, respectively. The $T_{1 / 2}$ values of the right kidney of group $B$ were significantly higher than those of group $A$ at all time points of obstruction. After relief of obstruction, the $T_{1 / 2}$ values returned back to their basal levels in both groups (Fig. 5). 

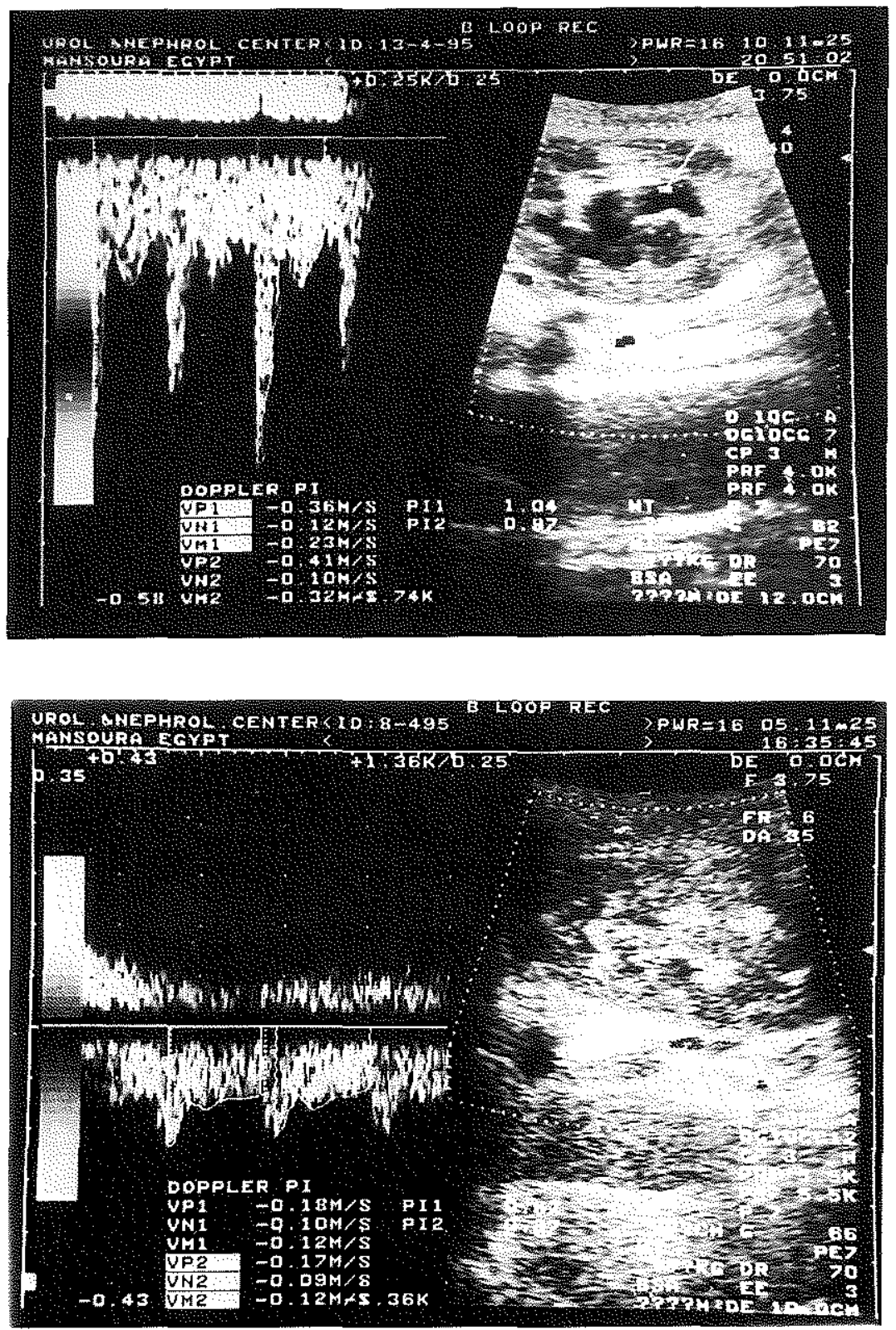

Fig. 3: Representative Doppler waveforms of a right kidney of one of the group A dogs: (A) 8 weeks after obstruction (mean resistive index $[R I]=0.64$ ), and $(B) 8$ weeks after release of obstruction (mean $\mathrm{RI}=0.44$ ). 


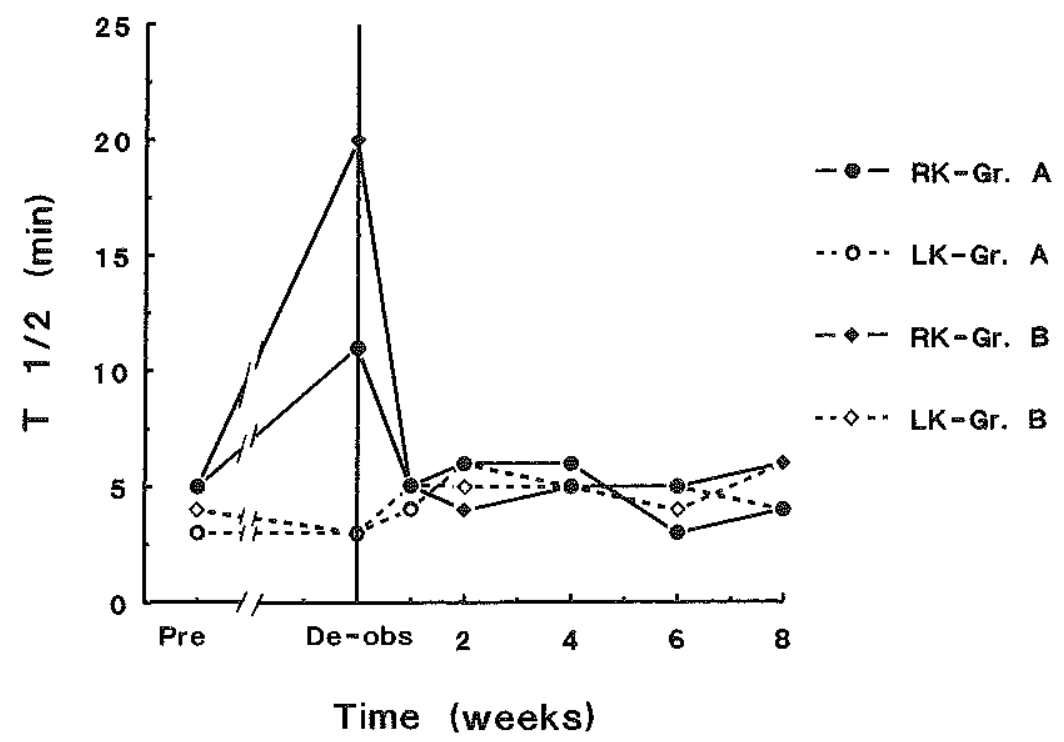

Fig. 4: Progress of the selective effective renal plasma flow (ERPF) of the corresponding (right) and contralateral (left) kidneys at different times of the study. (Closed circle: right kidney, group A; open circle: left kidney, group A; closed diamond, right kidney, group B; open diamond, left kidney, group B.)

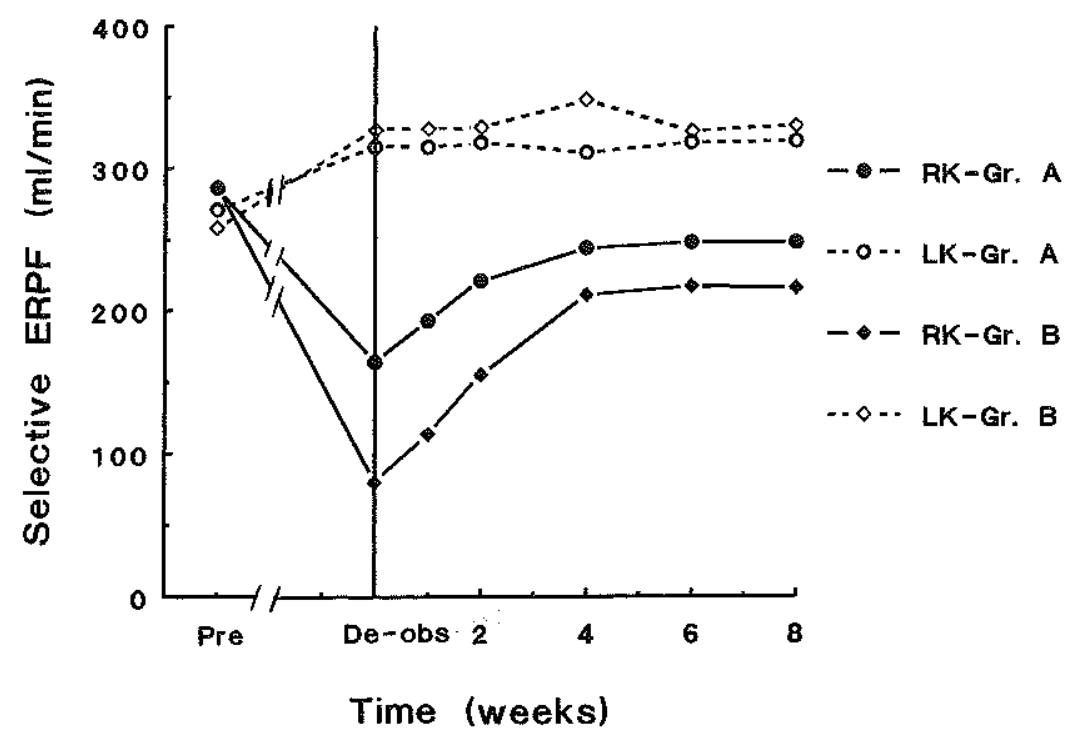

Fig. 5: Mean values of half-time drainage $\left(T_{1 / 2}\right)$ of both renal units at different times of the study. (Closed circle: right kidney, group $\mathrm{A}$; open circle: left kidney, group $\mathrm{A}$; closed diamond, right kidney, group B; open diamond, left kidney, group B.) 
Doppler ultrasonography studies of the left kidney

At basal condition, the mean RI values of the left kidney were $0.44 \pm 0.04$ and $0.47 \pm 0.06$ for groups $\mathrm{A}$ and $\mathrm{B}$, respectively. By the end of the eighth week of right ureteric obstruction, the RI of the left kidney decreased significantly to $0.40 \pm 0.03$ and $0.41 \pm 0.04$ for groups $\mathrm{A}$ and $\mathrm{B}$, respectively and remained stable after relief of obstruction (Fig.2). No significant differences were present between the mean levels of RI of groups A and B at any time.

Changes in ERPF of the left kidney

For group $A$, the mean ERPF of the left kidney at basal condition was $271 \pm 64 \mathrm{~mL} / \mathrm{min}$. By the end of the eighth week of obstruction of the right kidney the mean ERPF of the left kidney significantly increased to $315 \pm 58 \mathrm{~mL} / \mathrm{min}$. After relief of obstruction, the ERPF of the left kidney remained stable with mean values of $314 \pm$ 60 and $319 \pm 63 \mathrm{~mL} / \mathrm{min}$ at the end of the first and eighth week of deobstruction, respectively (Fig. 4).

For group $\mathrm{B}$, obstruction of the right kidney significantly increased the ERPF of the left kidney from a basal mean value of $258 \pm$ $67 \mathrm{~mL} / \mathrm{min}$ to $327 \pm 28 \mathrm{~mL} / \mathrm{min}$ at the end of the eighth week of obstruction. There was no significant change of the ERPF of the left kidney after relief of obstruction of the right kidney. At the end of the eighth week after release of obstruction, the ERPF of the left kidney was $330 \pm 53 \mathrm{~mL} / \mathrm{min}$ (Fig. 4).

\section{Changes in T1/2 of Left Kidney}

The $T_{1 / 2}$ of the left kidney remained stable during all phases of the experiments in both groups (Fig. 5).

\section{Changes in total ERPF}

The mean total ERPF values at basal condition were $557 \pm 68$ and $546 \pm 92 \mathrm{~mL} / \mathrm{min}$ for groups A and B, respectively. By the end of the eighth week of obstruction, there was significant reduction of the mean total ERPF values with the decrease being significantly more marked in group B ( $479 \pm 53$ versus $407 \pm 56 \mathrm{~mL} / \mathrm{min}, \mathrm{p}<0.05)$. After relief of obstruction, dogs of both groups regained their original function. However, regain of function was faster in group A. ( 4 weeks in group A versus 8 weeks in group B) (Fig. 6). 


\section{Correlations between the various parameters}

To find a potentially useful predictor of functional recovery after release of obstruction, we calculated the correlation coefficients between the ERPF of the previously obstructed kidney at 8 weeks after release of obstruction and various parameters that were obtained at the end of the obstruction period. Unfortunately, none of the measured parameters of the obstructed kidney (RI, ERPF, and $T_{1 / 2}$ ) nor parameters derived from it (the relative contribution to total ERPF or the obstructed/nonobstructed RI ratio) correlated $(p>0.05)$ with the ERPF value obtained following recovery after release of obstruction. This indicates that the magnitude of these parameters at the time of release of obstruction do not predict the functional recovery of the partially obstructed kidney. A more positive view is that with an ERPF as low as $12 \%$ of the basal value a good recovery is still possible. Actually, when entering basal ERPF of the obstructed kidney into the analysis, a positive correlation was found between the basal ERPF and the ERPF after recovery $(r=0.73, p=0.016)$. Multiple regression analysis showed that: ERPF recovery $=0.81 \times$ ERPF basal $+0.35 \times$ ERPF obstruction $43(\mathrm{~mL} / \mathrm{min})$. This indicated that the ERPF after release of obstruction depends more on basal ERPF than on the ERPF at the end of the obstruction period $(r=0.88, \mathrm{p}=0.05)$.

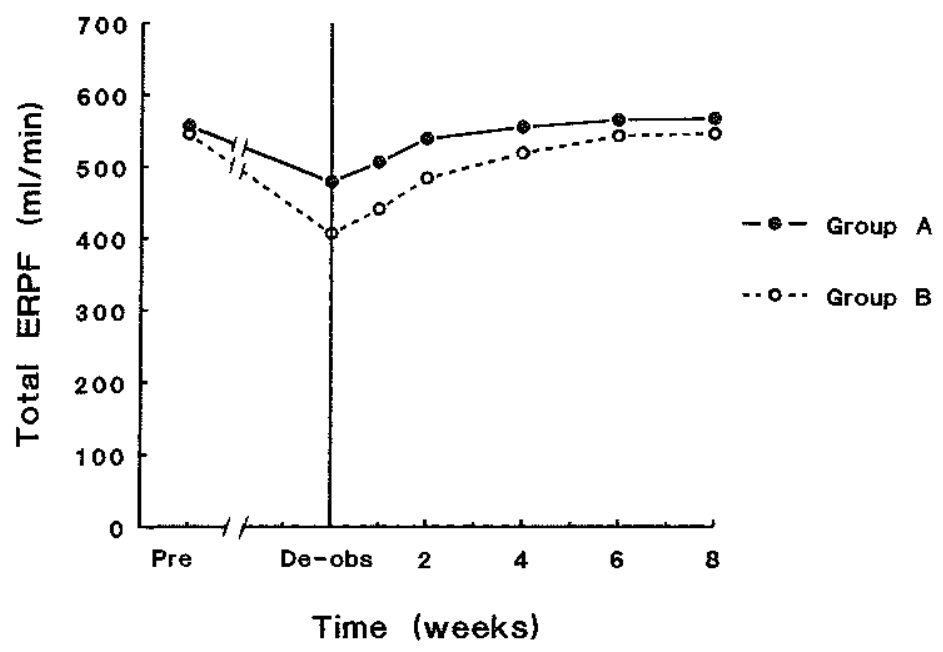

Fig. 6: Changes in total effective renal plasma flow (ERPF) of both kidneys at the different times of the study. (Closed circle, group A; open circle, group B.)

\section{Discussion}

Obstructive uropathy with resultant hydronephrosis is the eventual outcome of most urologic diseases. It is well known that 
ureteral obstruction eventually destroys renal function. The postulated mechanisms are elevated ureteral pressure and decreased renal blood flow, which cause cellular atrophy and necrosis. The longer or more severe the obstruction, the more renal damage will result.

The accurate prediction of recoverability of kidney function after obstructive uropathy is of great potential clinical value to the urologist and nephrologist. If restoration of kidney function appears probable, surgical relief of obstruction may be indicated even though there has been considerable renal damage. In patients whose kidney function is irreversibly damaged by the underlying obstructive process, it may be preferable to apply nonsurgical management or nephrectomy.

The recovery potential is dependent upon many things, most important of which are severity of renal injury and the presence or absence of infection. Great recovery is seen under the stimulus of impaired function of the opposite kidney (Kalika et al., 1981), In children, many urologists believe that the potential for recovery of what seems to be a severely damaged renal unit is great (Kalika et al., 1981).

Determination of recovery potential has historically been based on gross inspection or histologic evaluation at surgery or preoperatively by intravenous urography. Recently, renal scan with different tracers has been used to assess recoverability in hydronephrosis (Chibber et al., 1981; McDougal and Flanigan, 1981). Temporary relief of the obstruction with nephrostomy tubes followed by tests measuring renal function may be helpful in determining recoverability of function in hydronephrosis (Gillenwater et al., 1975).

There is an extending body of information which suggests that Doppler ultrasonography will be of increasing use in identifying clinically significant obstructive uropathy (Gilbert et al., 1993; Keller et al., 1989; Platt et al., 1991; Shokeir et al., 1996c). One of the remaining points of controversy is the exact role of RI in predicting recoverability of renal function after relief of ureteral obstruction. In the present study, reversal of RI to its basal value was associated with recovery of function after relief of obstruction in all renal units. Palmer and coworkers (1991) have shown an increase in renal RI in a group of children who subsequently underwent reconstruction. Fortunately, they were able to follow these children; in a second publication 2 years later, they demonstrated that, of the 9 kidneys measured 3 months postoperatively, all had a decrease in RI values less than 0.75 (Ordorica et al.,1993). Chen and associates (1993) have demonstrated a decrease of RI to below 0.70 in 3 of 5 patients after release of severe obstruction. The other 2 patients still had persistent elevated RI after relief of obstruction. This might be due to permanent parenchymal change because the serum creatinine levels remained unchanged during follow-up after relief of obstruction.

In the present study, recovery of ERPF could not be predicted from the changes of RI before release of obstruction. However, a 
reversal of a previously elevated RI could be used as an early indicator that recovery of ERPF is likely. This could be clinically applied by monitoring RI before and after temporary release of obstruction via percutaneous nephrostomy. In the future, we may be able to couple Doppler ultrasonography with the administration of vasoactive drugs to look for a reversal of a high $\mathrm{Rl}$ to predict recovery following corrective surgery (Vaughan, 1995).

Most investigators have agreed that marked partial ureteral obstruction results in an increase of intrarenal vascular resistance that is reflected as an elevation of PI and RI. The increase of vascular resistance in mild partial ureteral obstruction is still a matter of controversy. Although this concept has been supported by some (Ryan et al., 1987; Restaino et al., 1990), it has been questioned by others (Chevalier and Jones, 1986). Differences in results could be explained by the difference in the definition of the mild obstruction. This study helps to solve this issue. We have demonstrated that a significant increase in $\mathrm{Rl}$ is present 2 weeks after induction of mild partial ureteral obstruction in a standard and stable experimental model.

Our observation that the RI of the contralateral normal kidney is decreased after obstruction of the other kidney may be explained by a decrease in renal vascular resistance of the contralateral kidney due to compensatory functional and structural adaptations (lchikawa and Brenner, 1979). The human equivalent could be explored through a study of a group of live-kidney donors before and after nephrectomy.

It has been estimated that the value of RI of 0.70 is the dividing line between obstructive and nonobstructive dilated upper urinary tracts. The accuracy of this discriminatory value can be improved by evaluation of the contralateral kidney, especially in the acute obstruction in which the RI may not yet have exceeded 0.70. Platt et al. (1991) have suggested that if there is a greater than 0.10 difference between the obstructed and the contralateral kidney, this potentiates the diagnosis of obstruction. We have observed that unilateral obstruction produces an elavation of RI of the corresponding kidney and a decrease of RI of the contralateral kidney. This divergent response may be more valuable in the diagnosis of obstruction than a single discriminatory value.

At present, the diuretic renogram is used at most centers as the reference standard for diagnosis of obstruction (O'Reilly et al., 1978; Koff et al., 1980; Kass et al., 1985). An added benefit to radioisotope renography is the ability to measure differential glomerular filtration rate, thus arriving at a percentage of total glomerular filtration rate contributed by each kidney. The calculation of half-time drainage is more objective in the diagnosis of obstruction. Those kidneys able to excrete more than half of the radioisotope in less than 10 minutes against an empty bladder are considered nonobstructed and those requiring greater than 20 minutes are considered obstructed. We have 
demonstrated that RI values correlate closely with T1/2 values both in severe and mild partial ureteral obstruction. This means that RI is a useful modality in the evaluation of hydronephrosis even in its early and mild phases. These results are in agreement with those obtained by Gilbert and associates (1993). In their study, renal Doppler ultrasound findings were compared with drainage values obtained with renal scintigraphy diuretic renography in 28 hydronephrotic pediatric kidneys, and they demonstrated close correlation. On the other hand, Chen et at (1993) stated that not all renal obstructions have significantly elevated RI values. In cases of mild obstruction there may be low and normal RI values.

The renal Doppler examination represents physiologic information and does not suffice if precise anatomical information is required (Gilbert et al., 1993). Therefore, it cannot be used as a single modality of evaluation of a patient with hydronephrosis. Thus, the value of using the RI is that it provides corroboration of the initial diuretic renogram and a noninvasive modality for monitoring the dilated collecting system under observation. A hydronephrotic kidney with a non-obstructive $T_{1 / 2}$ on diuretic renography can be followed closely with surveillance renal Doppler ultrasound, obviating the need for frequent isotope renal scintigraphy. Additionally, this method provides a noninvasive modality for monitoring patients after reconstructive surgery of the upper urinary tract.

We could demonstrate that the rate and magnitude of compensatory response to mild obstruction (group A) was approximately the same as the response to severe obstruction (group B). This confirms the speculation that the progression of compensatory renal growth is an all-or-nothing phenomenom. If the threshold amount of kidney tissue is damaged or removed, then compensatory renal growth begins and progresses until compensatory growth is completed (Mitchell et al., 1974).

It has been shown from this experiment that if ureteral obstruction is relieved after 8 weeks, the post-obstructed kidney will gradually improve its function, regardless of the degree of partial obstruction. As expected, hypertrophy occurs in the opposite kidney in response to the obstruction. Several studies note that the contralateral hypertrophy will persist after the obstruction is relieved and that total renal function will be better in such a situation than if the obstructed kidney had been removed. This is true even if the affected kidney involved has been severely obstructed. This argues that, in general, even with compensatory renal growth considered, total renal function is maximized by unobstructing any functioning kidney (Stecker and Gillenwater, 1971). 
Renal Doppler ultrasound in children with normal upper urinary tracts: effect of fasting, hydration with normal saline, and furosemide administration.

Ahmed A. Shokeir, Abraham P. Provoost, Mohamed El-Azab, Mohamed Dawaba and Rien J. M. Nijman

Urology and Nephrology Center, Mansoura University, Mansoura, Egypt, and Departments of Pediatric Surgery and Pediatric Urology, Erasmus University, Rotterdam, The Netherlands

Urology (1996) $47: 740-744$ 



\section{Abstract}

\section{Objectives}

To study the age dependency of renal resistive index (RI) and to study the effect on the renal RI of fasting, intravenous infusion of normal saline, and administration of furosemide in children with normat upper urinary tracts.

\section{Methods}

The study included 28 non-obstructed renal units in 15 boys ranging in age from 3 to 11 years. Diuretic renography and Doppler ultrasonography were attempted in all children. Doppler ultrasonography was carried out under three different conditions: fasting state, 30 to 60 minutes after intravenous infusion of normal saline $(15 \mathrm{~mL} / \mathrm{kg})$, and 10 minutes after administration of furosemide $(1 \mathrm{mg} / \mathrm{kg} ;$ maximum, $40 \mathrm{mg})$.

Results:

There was an inverse correlation between age and RI of both renal units under the three conditions of Doppler studies. At fasting state mean RI was $0.70 \pm 0.04$, whereas 15 of 28 renal units $(54 \%)$ had an RI of 0.70 or higher. Intravenous infusion of normal saline significantly decreased the RI to $0.63 \pm 0.04(p<0.000001)$. Injection of furosemide caused a further significant decrease of RI from $0.63 \pm 0.04$ to $0.60 \pm$ $0.04(\mathrm{P}<0.002)$.

\section{Conclusions}

The renal $\mathrm{RI}$ in healthy children is age dependent. In the fasting state, $54 \%$ of non-obstructed renal units in children have an RI of 0.70 or higher. Intravenous infusion of normal saline and administration of furosemide can independently cause a significant decrease of the $R I$ in non-obstructed renal units in children.

\section{Introduction}

In 1989, Platt et al. described the use of Doppler ultrasound to differentiate obstructive from non-obstructive pyelocaliectasis. A mean resistive index $(\mathrm{Rl})$ value of 0.70 has been used as the dividing line between obstruction and non-obstruction. Although an RI of 0.70 is 
accepted by most investigators as the higher level of normal in the adult population, more work needs to be done to establish accepted standards of normal and abnormal for pediatric renal Doppler waveforms. It has been shown that Doppler waveforms are likely to be age dependent, particularly in infants (Gilbert et al., 1993; Wong et al., 1989; Dejeter et al., 1988; Keller, 1989; Bude et al., 1992). Bude and associates (1992) have found that the renal RI in healthy children is commonly elevated above the upper limit in adults in the first year of life, and the overall trend shows a decrease with age. From 4 years on, the likelihood is low ( $2 \%$ probability) that the $\mathrm{RI}$ is above 0.70 . The $\mathrm{Rl}$ is also affected by some non-urologic renal diseases (Platt et al., 1991), oral hydration (Palmer et al., 1991), and the intravenous infusion of normal saline and furosemide in combination (Bude et al., 1994).

We studied the changes of renal vascular resistance in terms of RI under three different conditions in a group of children with normal upper urinary tracts. The RI was monitored in the fasting state, after intravenous infusion of normal saline, and after administration of furosemide. The study is important to define the normal values of RI under various conditions and may be useful in the development of a pharmacologically based Doppler sonographic method of evaluation of pyelocaliectasis.

Recent literature has shown that the combination of intravenous normal saline and furosemide can cause a significant decrease of RI in children with non-obstructed renal units (Bude et al., 1994). However, they could not know with certainty whether this decrease was due to saline only or due to saline and furosemide in combination. The present study is important to solve this issue.

\section{Material and methods}

The study included 15 male children with hypospadias with a mean age of 6.4 years (range, 3 to 11). Diuretic renography and Doppler sonography were attempted in all children during their stay in the hospital after correction of hypospadias. In all patients the urinary bladder was drained by a suprapubic catheter. The procedures were explained and informed consent was obtained from the parents.

Technetium-99m mercaptoacetyltriglycine (MAG3) diuretic renography was performed in all patients according to our standard protocol (Shokeir et al., 1994). Doppler ultrasonography was carried out on a subsequent day early in the morning after the child had been fasting for 10 to 12 hours. The child was then allowed to drink and a bolus dose of intravenous normal saline $(15 \mathrm{~mL} . / \mathrm{kg}$.) was given at a rate of 1 $\mathrm{mL} / \mathrm{kg} / \mathrm{min}$ and Doppler study was repeated. A third Doppler study was 
carried out 10 minutes after injection of furosemide (1 $\mathrm{mg} / \mathrm{kg}$; maximum, $40 \mathrm{mg}$.). An interval of I hour was given between the second and third Doppler studies during which the maximum effect of intravenous fluid load had started to decrease. Intravenous normal saline was infused during the entire second and third Doppler studies to replace urine output.

Ultrasound examinations were performed on a Toshiba (SSA$270 \mathrm{~A}$ ) unit using transducer frequency of $3.75 \mathrm{MHz}$. Renal morphology was studied in longitudinal and transverse planes. At least 5 Doppler spectra were obtained from more than three regions in each kidney in every study. Renal pulsatility indexes (PIs) were obtained using Toshiba software from the formula: $\mathrm{PI}=$ (peak systolic velocity - end diastolic velocity)/mean velocity. The renal RIs were calculated as: $\mathrm{RI}=$ (peak systolic velocity - end diastolic velocity) / peak systolic velocity. Values of $\mathrm{Pl}$ and RI used in statistical analyses were averages of the mean measurements of each study in the individual kidney.

Statistical analyses were performed with paired and unpaired $t$ test, chi-square test, and simple linear regression analysis.

\section{Results}

Two of the 15 children had solitary renal units. The mean selective renographic MAG3 clearance was $53 \pm 5$ and $52 \pm 6 \mathrm{~mL} / \mathrm{min}$ for the right and left kidneys, respectively (corrected for body surface area). The mean half-time drainage of the right kidneys was $5.6 \pm 2.5$ and that of the left kidneys was $4.6 \pm 2.4$ minutes.

Satisfactory Doppler examinations were obtained in all 28 renal units and the results are summarized in Table 1. Representative waveforms under the three conditions of Doppler examinations are illustrated in Figure 1. There was excellent positive correlation between Pl and RI during the whole phases of Doppler studies (correlation coefficient $=0.83 ; \mathrm{P}=0.0001 ;$ Figure 2). Because the PI and Rl were positively correlated, all results of the RI were also applicable to PI. For this reason, we only discussed the changes of vascular resistance in terms of RI. Results of the right kidneys were comparable to those of the left kidneys during the three phases of Doppler studies (Table 1). 


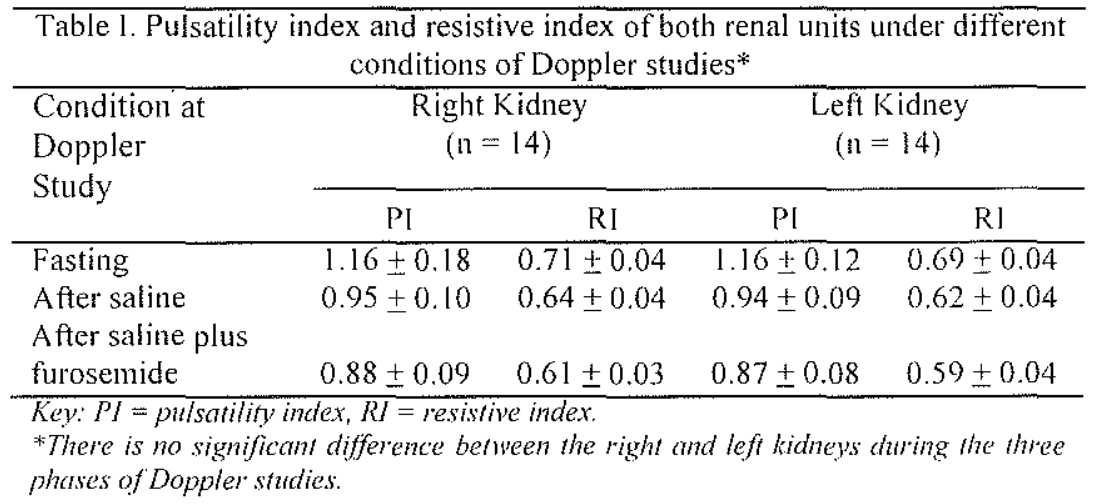

Children were stratified into two groups according to their ages: those with an age less than 4 years ( 7 children with 13 renal units) and those 4 or more years old ( 8 children with 15 renal units). After infusion of normal saline, the RI was found to be significantly higher in the group of children with younger age $(\mathrm{P}<0.001)$ (Table II). Moreover , there was an inverse correlation between age and $\mathrm{RI}$ of both renal units under the three conditions of Doppler studies (Fig. 3).

Figure 4 illustrates the mean and standard deviation of PI and $\mathrm{RI}$ of all renal units at the three phases of Doppler studies. RI values of $0.70 \pm 0.04,0.63 \pm 0.04$, and $0.60 \pm 0.04$ were obtained at fasting state, 30 to 60 minutes after intravenous infusion of normal saline, and 10 minutes after administration of furosemide, respectively. A significant decrease could be observed comparing the RI at fasting state and following saline infusion ( $\mathrm{P}<0.000001)$. Comparison of the $\mathrm{Rl}$ after saline infusion with that after furosemide injection also revealed a statistically significant difference $(\mathrm{P}<0.002)$.

\begin{tabular}{|c|c|c|c|}
\hline \multirow[b]{2}{*}{$\begin{array}{l}\text { Condition at Doppler } \\
\text { Study }\end{array}$} & \multicolumn{2}{|c|}{ RI (nean \pm SD) } & \multirow[b]{2}{*}{$\begin{array}{c}\text { P } \\
\text { Value }\end{array}$} \\
\hline & $\begin{array}{l}<4 \text { years } \\
\text { (13 units) }\end{array}$ & $\begin{array}{l}\geq 4 \text { years } \\
\text { (15 units) }\end{array}$ & \\
\hline Fasting & $0.70 \pm 0.04$ & $0.69 \pm 0.05$ & NS \\
\hline After saline & $0.65 \pm 0.04$ & $0.61 \pm 0.03$ & 0.001 \\
\hline After saline plus furosemide & $0.61 \pm 0.04$ & $0.59 \pm 0.04$ & NS \\
\hline
\end{tabular}

Key: $N S=$ not significant: $R I=$ resistive index 

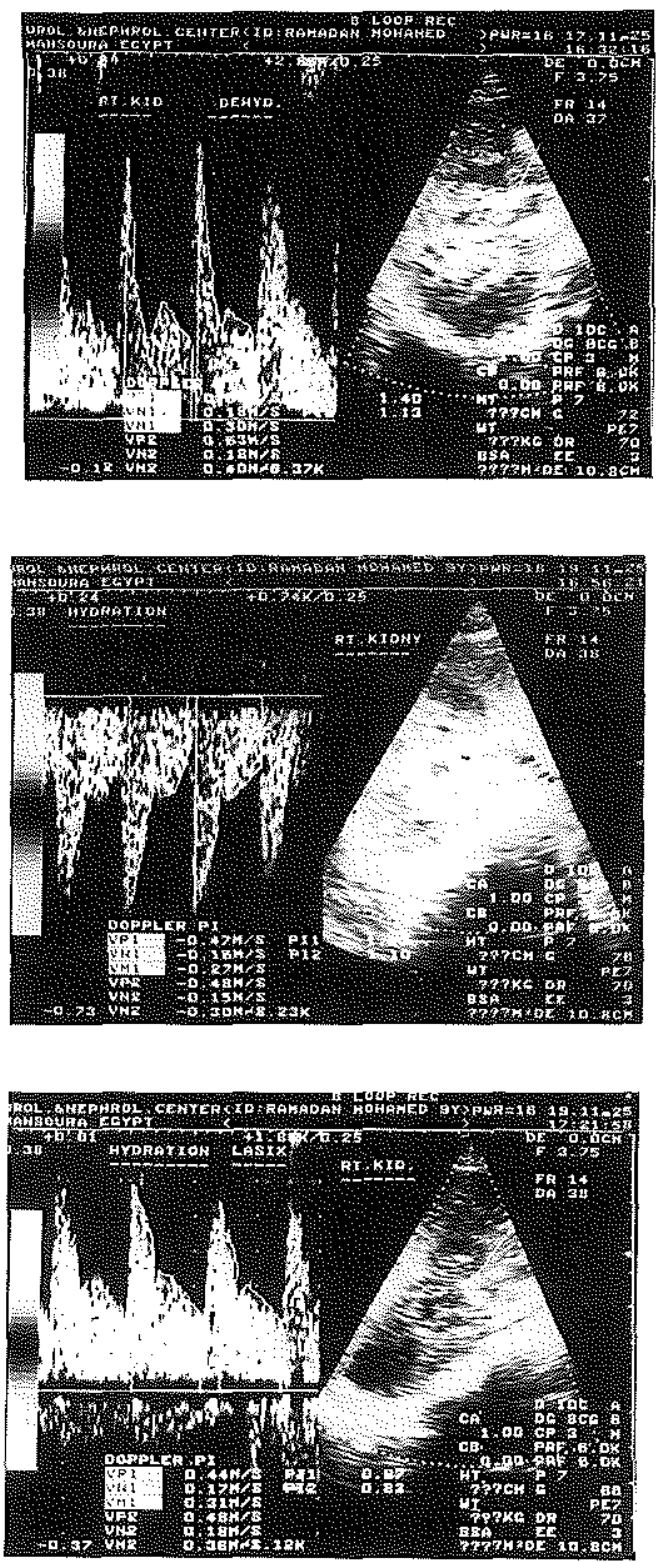

Fig. 1: Doppler waveforms of the sane kidney in the same patient at (A) fasting state (mean pulsatility index [Pl], 1.27; mean resistive index [RI], 0.71), (B) alter intravenous infusion of normal saline (mean PI, 1.13; mean RI, 0.68), and (C) after injection of furosemide (mean PI, 0.85; mean RI, 0.62). 


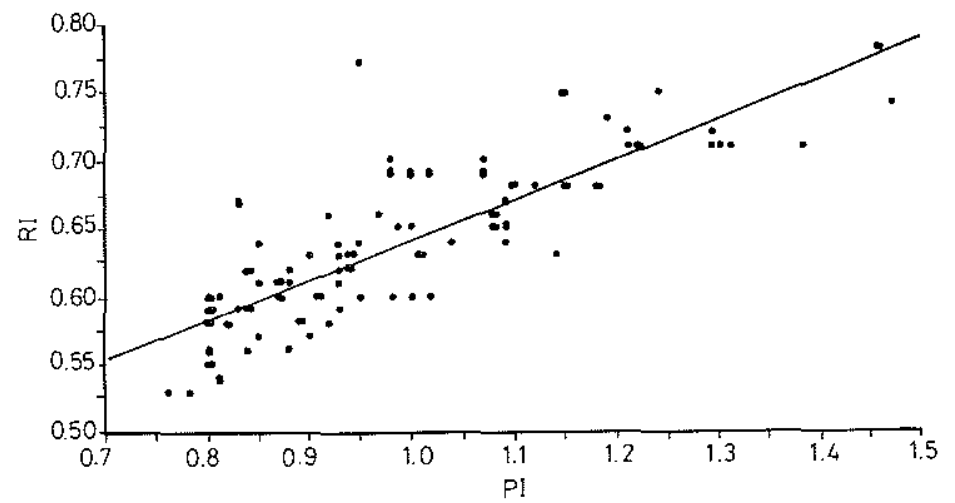

Fig. 2: Correlation between pulsatility index (PI) and resistive index (RI) during the entire three phases of the study $(r=0.83 ; \mathrm{P}=0.0001)$.

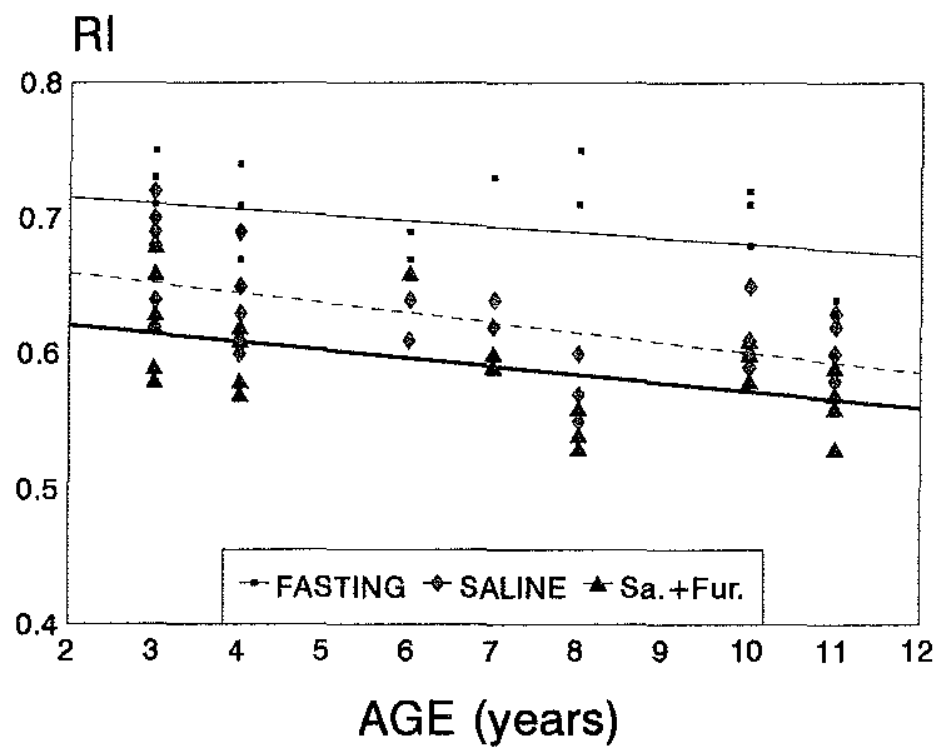

Fig. 3: Correlation between age and resistive index (RI) of both renal units at fasting state $(R=-0.41 ; P=0.04)$, after infusion of normal saline ( $\mathrm{Sa} .: R=-0.58, P=0.0008)$, and after administration of furosemide (Fur.; $R=-0.47, P=0.008$ ) 


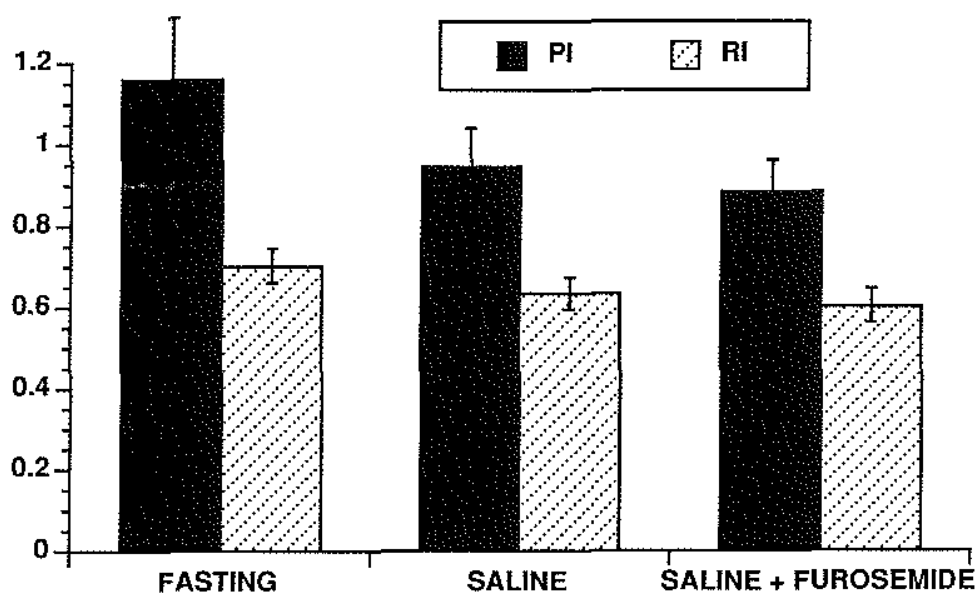

Fig. 4: Mean and standard deviation of pulsatility index (PI) and resistive index (RI) during the three phases of the study.

At fasting state, 15 of 28 renal units $(54 \%)$ had an $\mathrm{RI}$ value of 0.70 or higher. On the other hand, none of the renal units had an RI of 0.70 or higher after infusion of normal saline and administration of furosemide, a difference of statistical significance $(P=0.0000004)$ (Table III).

\begin{tabular}{lc}
\hline $\begin{array}{c}\text { Table III : Renal units with resistive index of } 0.70 \text { or higher under the three conditions } \\
\text { of Doppler studies }\end{array}$ \\
\hline $\begin{array}{c}\text { Condition at Doppler Study } \\
\text { Nonal units with RI of } 0.70 \text { or } \\
\text { higher }(\%)^{*}\end{array}$ \\
\hline $\begin{array}{l}\text { Fasting } \\
\text { Saline }\end{array}$ & $15(54 \%)$ \\
& 0 \\
\hline
\end{tabular}

Key $: \mathrm{R} \mid=$ resistive index

$* X 2=29.35 ; p=0.000004$.

\section{Comment}

In the past, ultrasonography was criticized because of its inability to help differentiate clearly between obstructed and nonobstructed urinary tracts. This dilemma has been addressed with other examinations, such as diuretic radionuclide renography and pressure flow studies (Whitaker, 1973; Jaffe and Middleton, 1980). A renewed interest in ultrasonography to differentiate between obstructive and nonobstructive dilation of the upper tract was addressed by Platt et al in 1989. They reported their experience with renal artery waveform 
analysis in kidneys with dilated collecting systems before and after percutaneous nephrostomy. In their series of adults, an RI of 0.70 emerged as the dividing line between obstructive and non-obstructive dilation. This concept holds promise for future clinical application in both adults and children. However, more work needs to be done to establish accepted standards of normal and abnormal values for pediatric renal Doppler waveforms (Keller, 1989).

It has recently been shown that the $\mathrm{RI}$ is age dependent. It is frequently elevated above 0.70 in children younger than I year and can sometimes be greater than 0.70 before the age of 4 years (Gilbert et al., 1993; Wong et al., 1989; Dejeter et al., 1988; Keller, 1989; Bucle et al., 1992). In this study, it has been shown that there is an inverse correlation between age and $\mathrm{RI}$ and that after hydration the RI is higher among children younger that 4 years compared with older children. Possible explanations for higher $\mathrm{Rl}$ in younger children are increased renal vascular resistance and decreased total blood flow in the infant kidney (Gilbert et al., 1993). However, Palmer et al.(1991) found no inverse relationship between age and $\mathrm{RI}$ in children. Further research in the pediatric age group is needed to define the age at which the kidneys assume adult renal resistance levels.

A noteworthy observation in this series is that the RI is 0.70 or higher in $54 \%$ of non-obstructed renal units in fasting children. After hydration, the RI regains its normal value previously mentioned in the literature. This observation addresses the importance of at least oral hydration for proper interpretation of Doppler studies. It is evident that changes in RI after hydration or furosemide administration or both are more accurate for predicting renal obstruction.

Certain types of renal medical diseases also cause elevations of RI, such as acute tubular necrosis, interstitial nephritis, and hemolyticuremic syndroms (Platt et al., 1991). Therefore, criteria predicting obstruction based on a threshold level of 0.70 may be meaningless in the pediatric population. Bude and associates (1994) have addressed the idea of a test that would identify obstruction, not based on a single basal RI value, but on a change in RI through a standardized pharmacologic test. For this test, they suggested the use of intravenous normal saline and furosemide. In their recent study, they have demonstrated that the combination of intravenous normal saline and furosemide caused a significant decrease of RI in 13 non-obstructed renal units. However, they could not know for certain whether this decrease was due to saline only or due to saline and furosemide in combination. The present study has solved this issue and showed that intravenous administration of normal saline as well as furosemide both significantly decrease the RI in non-obstructed kidneys. Other studies, however, showed that furosemide has no effect on the RI in non-obstructed kidneys in children (Keller 1989; Palmer et al., 1991) and in adults (Renowden and Cochlin, 
1992). In these latter studies, however, furosemide was used alone without intravenous normal saline.

Our observation that intravenous administration of normal saline and furosemide can decrease the RI in non-obstructed kidneys is potentiated by the physiologic fact that both normal saline and furosemide can decrease renal vascular resistance (Hook et al., 1966; Gerber and Nies, 1980; Ludens et al., 1968). Ludens and associates (1968) demonstrated a significant lowering of the renal vascular resistance in dogs after intravenous infusion of normal saline and furosemide. This decrease in renal vascular resistance was dose related. When the normal saline dosage was maintained constant, larger doses of furosemide decreased the vascular resistance. Similarly, with constant doses of furosemide, increased doses of saline decreased the vascular resistance. It is likely that a similar decrease in renal vascular resistance is reflected in the decrease of renal RI in our series.

Although obstructed kidneys are not included in this study, it is expected that the use of intravenous normal saline and furosemide will cause an increase of RI of obstructed kidneys. This speculation is based on the previous reports that the use of furosemide alone can cause significant increase of RI of obstructed kidneys (Palmer et al., 1991; Renowden and Cochlin, 1992). If this is true, the use of saline and furosemide would produce a divergent effect, causing the RI to increase in obstructed kidneys and to decrease in non-obstructed ones. Such a divergent response might be useful in the diagnosis of partial unilateral kidney obstruction by exaggeration of the difference between kidneys. A current study investigating the effect of infusion of normal saline and administration of furosemide on the renal RI of children with obstructed renal units is ongoing. 

Renal Doppler ultrasound in children with obstructive uropathy: effect of intravenous normal saline fluid load and furosemide.

Ahmed A. Shokeir, Abraham P. Provoost, Mohamed El-Azab, Mohamed Dawaba and Rien J. M. Nijman.

Urology and Nephrology Center, Mansoura University, Mansoura, Egypt, and Departments of Pediatric Surgery and Pediatric Urology, Erasmus University, Rotterdam, The Netherlands

J. Urol. (1996 ) 156: 1455-1458 



\section{Abstract}

Purpose

We studied the effect of hyperhydration with normal saline and furosemide on renal resistive index in children with obstructive uropathy.

\section{Materials and Methods}

99m Technetium-mercaptoacetyltriglycine diuretic renography and Doppler ultrasound were done in 27 children (54 renal units) with unilateral or bilateral hydronephrosis. Doppler studies were performed at baseline, and after infusion of normal saline and administration of furosemide. Half-time drainage, considered the gold standard for the diagnosis of renal obstruction, was compared to resistive index.

Results

There was a positive correlation between half-time and resistive index on both Doppler studies. With a resistive index of 0.70 as the critical value for predicting renal obstruction 82 versus $100 \%$ sensitivity $(p<0.006), 63$ versus $94 \%$ specificity $(p<0.04)$ and 76 versus $98 \%$ overall accuracy $(\mathrm{p}<0.0005)$ were obtained for Doppler studies at baseline and after induced diuresis, respectively. All children with falsepositive results were younger than age 4 years.

\section{Conclusion}

Doppler ultrasonography after hyperhydration with normal saline and furosemide is an accurate method for diagnosing renal obstruction in children. It is more sensitive, specific and accurate than baseline Doppler studies.

\section{Introduction}

In 1989 Platt et al. described performing Doppler ultrasound to differentiate obstructive from non-obstructive pyelocalicectasis. Although a resistive index of 0.70 is accepted by most investigators as a high normal level in the adult population, more studies must be done to establish accepted standards of normal and abnormal values for pediatric renal Doppler waveforms. It has been shown that Doppler waveforms are likely to be age dependent, particularly in infants. (Gilbert et al., 
1993; Wong et al., 1989; Bude et al., 1989; Keller et al., 1989). Resistive index is also affected by some medical renal diseases (Platt et al., 1991), oral hydration (Palmer et al., 1991), and the combination of intravenous normal saline and furosemide (Bude et al., 1994). Resistive index is frequently elevated above 0.70 in children and in some renal diseases, such as acute tubular necrosis, interstitial nephritis and the hemolytic-uremic syndrome. Therefore, predicting obstruction based on a threshold of 0.70 may be meaningless in children. We evaluated the effect of normal saline fluid load and furosemide to determine whether they caused any significant improvement in the diagnostic accuracy of a resistive index value of 0.70 as a predictor of renal obstruction in children.

\section{Patients and methods}

We evaluated intra-renal hemodynamics in 33 children with unilateral or bilateral obstructive uropathy on Doppler ultrasonography. Hydronephrosis was diagnosis by ultrasound and excretory urography, and confirmed by diuretic renography with determination of half-time drainage (the time necessary for half of the radioisotope to be eliminated from the pelvicaliceal system). Kidneys with a half-time less than 10 minutes were considered non-obstructed and those with a half-time greater than 20 minutes were considered obstructed. Six patients with an indeterminate half-time (between 10 and 20 minutes) were excluded from study due to inadequate data on the degree of renal obstruction. A total of 16 boys and 11 girls (54 renal units) 1 to 12 years old (mean age plus or minus standard deviation $5.7 \pm 3.4$ ) was eligible for study. In all patients hydronephrosis was discovered during childhood. Obstruction was bilateral in 11 patients and unilateral in 16. The causes of obstruction were ureteropelvic junction obstruction in 29 renal units, posterior urethral valves in 10 and urolithiasis in 15 . In the 16 patients with unilateral obstruction the contralateral kidney was normal ( 8 renal units) or dilated and non-obstructed (8) with a half-time less than 10 minutes. The causes of non-obstructive dilatation were non-refluxing megaureter in 3 renal units and non-obstructive ureteropelvic junction narrowing in 5 .

All children were well hydrated before sonography and indwelling catheters were placed to ensure the absence of bladder pressure artifact. Doppler ultrasonography was done at baseline and repeated after intravenous infusion of $15 \mathrm{ml} / \mathrm{kg}$. normal saline during 30 to 60 minutes, followed by an injection of $1 \mathrm{mg} . / \mathrm{kg}$. furosemide (maximum $40 \mathrm{mg}$.). The study was repeated 10 minutes after the 
furosemide injection. Infusion of normal saline continued during the entire second Doppler study to replace urine output.

Ultrasound was performed on a Toshiba SSA-240A unit using a transducer frequency of $3.75 \mathrm{MHz}$. Renal morphology was studied in longitudinal and transverse planes. At least 5 Doppler spectra were obtained from more than 3 regions in each kidney in every study. Renal pulsatile index (PI) was determined using computer software and the formula, PI = (peak systolic velocity - end diastolic velocity) / mean velocity. Renal resistive index was calculated using the formula, $\mathrm{RI}=$ (peak systolic velocity- end diastolic velocity) / peak systolic velocity. Values of pulsatile and resistive indexes used for statistical analysis were averages of the mean measurements of each study in the individual kidneys.

Diuretic renography was done according to a standard protocol in all patients. A urethral catheter was placed, an intravenous line was established and $15 \mathrm{ml} / \mathrm{kg}$. normal saline were given. The patient was placed supine, $57 \mathrm{uCi} . / \mathrm{kg}$. ${ }^{2 \% \mathrm{~m}}$ technetium-mercaptoacetyltriglycine were injected intravenously and a standard renal scan was preformed. After 20 minutes $1 \mathrm{mg} . / \mathrm{kg}$. furosemide was injected intravenously. Sequential images were obtained by a gamma camera computer system for 30 minutes, and the data were processed for generation of a time activity curve and calculation of glomerular filtration rate and half-time. Our technique of diuretic renography has been previously described. (Shokeir et al., 1994)

\section{Results}

Of the 54 renal units 38 were obstructed and 16 were nonobstructed ( 8 normal and 8 dilated). Glomerular filtration rate, half-time and resistive index values of obstructed, dilated non-obstructed and normal renal units are shown in table 1 . Values of dilated nonobstructed and normal renal units were comparable. On the other hand, obstructed rena! units had a significantly lower glomerular filtration rate, longer half-time and higher resistive index compared to nonobstructed renal units.

There was positive correlation between half-time and resistive index in all renal units at baseline $(r=0.8, p<0.0001)$ and after the infusion of normal saline and furosemide $(r=0.9, p<0.00001$, fig 1 , A). Furthermore, there was an inverse correlation between glomerular filtration rate and resistive index in all renal units on both Doppler studies (fig. 1,B). Half-time, considered the gold standard for the diagnosis of obstruction, was assessed as positive when greater than 20 minutes and negative when less than 10 minutes. Resistive index was compared to half-time, and was considered positive (sensitivity) when 
resistive index was greater than 0.70 and half-time was greater than 20 minutes. Doppler studies were considered negative (specificity) when resistive index was less than 0.70 and half-time was less than 10 minutes.

Table 1 . Summary of the results of obstructed and non-obstructed renal units

Obstructed Non-obstructed
(p Value not significant)

\begin{tabular}{|c|c|c|c|c|}
\hline & & Dilated & Normal & Overall \\
\hline No. renal units & 38 & 8 & 8 & 16 \\
\hline GFR (ml./min.) $)^{*}$ & $21 \pm 10.5$ & $54 \pm 6.7$ & $56 \pm 7.2$ & $55 \pm 6.9$ \\
\hline Half-time (min.) ${ }^{*}$ & $26.6 \pm 4.4$ & $6.8 \pm 1.7$ & $5.5 \pm 2.0$ & $6.0 \pm 1.9$ \\
\hline \multicolumn{5}{|l|}{ Resistive index * } \\
\hline At baseline & $0.75 \pm 0.06$ & $0.66 \pm 0.04$ & $0.66 \pm 0.05$ & $0.66 \pm 0.04$ \\
\hline $\begin{array}{l}\text { After saline \& } \\
\text { furosemide }\end{array}$ & $0.79 \pm 0.06$ & $0.60 \pm 0.05$ & $0.61 \pm 0.05$ & $0.60 \pm 0.05$ \\
\hline
\end{tabular}

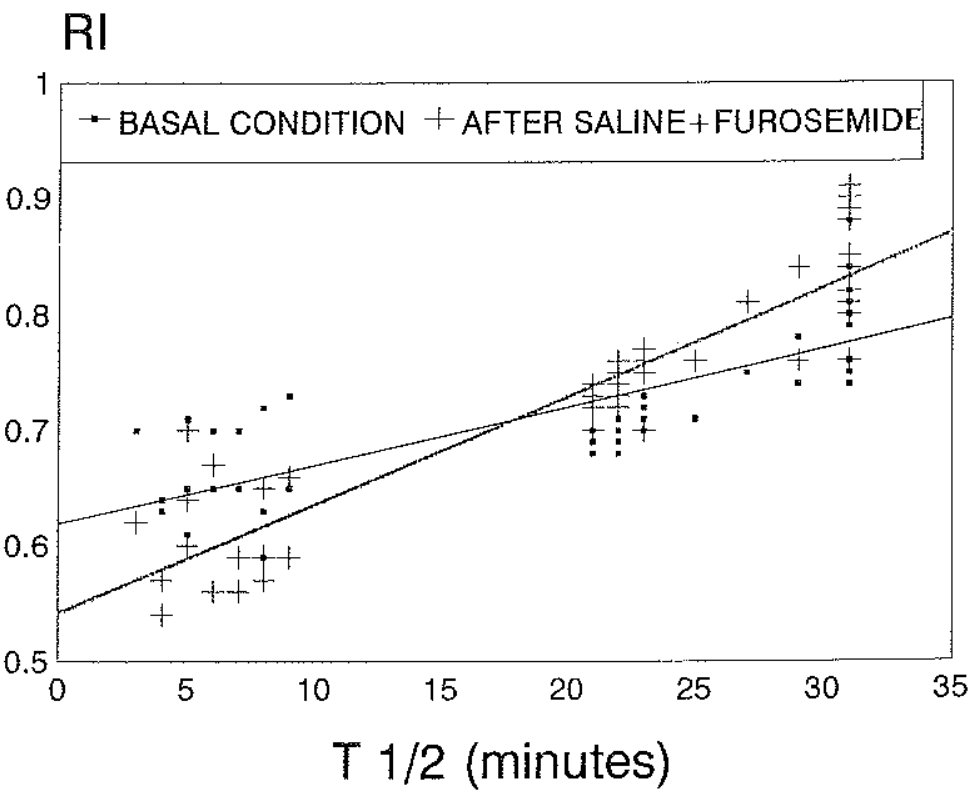

Fig. 1: A, correlation between half-time $\left(\mathrm{T}_{U 2}\right)$ and resistive index $(\mathrm{RI})$ at baseline $(\mathrm{r}=0.8$, $p<0.000 \mathrm{I})$, and after addition of saline and furosemide $(r=0.9, p<0.00001)$. 


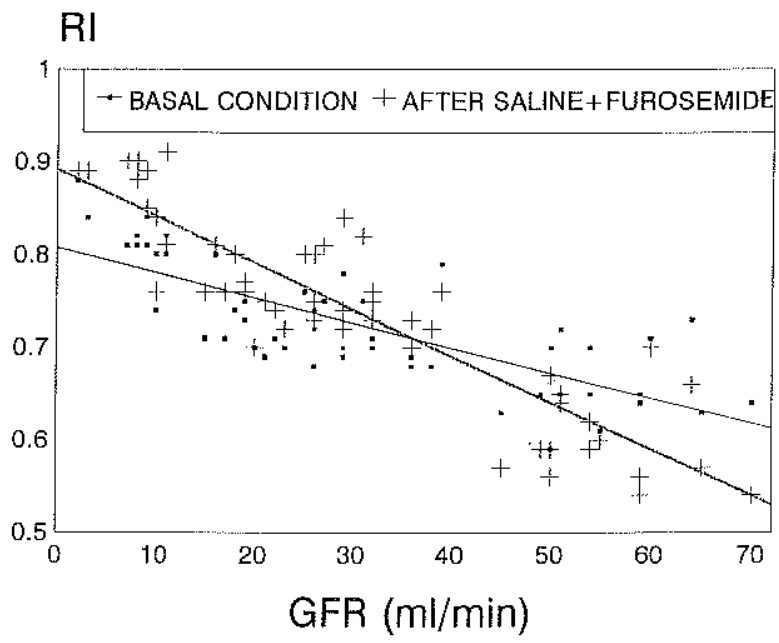

Fig. 1: B, correlation between glomertlar filtration rate (GFR) and resistive index at baseline $(r=-0.8, p<0.000)$, and after addition of saline and furosemide $(r=-0.9, p<$ $0.00001)$.

At baseline mean resistive index plus or minus standard deviation was $0.66 \pm 0.04$ and $0.75 \pm 0.06$ in non-obstructed and obstructed kidneys, respectively. With a resistive index of 0.70 as the critical value for predicting renal obstruction sensitivity was $82 \%$, specificity $63 \%$ and overall accuracy $76 \%$ (table 2 ). After the infusion of normal saline and administration of furosemide there was a significant increase in the resistive index of obstructed kidneys $(0.75 \pm 0.06$ versus $0.79 \pm 0.06, p<0.001)$ and a significant decrease in the resistive index of non-obstructed kidneys $(0.66 \pm 0.04$ versus $0.60 \pm 0.05, \mathrm{p}=0.0006)$. This divergent response improved the results, and values of 100,94 and $98 \%$ were obtained for sensitivity, specificity and overall accuracy, respectively (table 2). A comparison of sensitivity, specificity and overall accuracy on both Doppler studies is shown in table 3. Diuretic Doppler ultrasonography was significantly more sensitive, specific and accurate for predicting renal obstruction than Doppler ultrasonography at baseline. Representative Doppler waveforms of an obstructed and a normal kidney before and after induction of diuresis are shown in figures 2 and 3, respectively. 
Table 2. Agrcement between half-time \& resistive index

\begin{tabular}{llcc}
\hline & $\begin{array}{c}\text { Resistive } \\
\text { Index }\end{array}$ & \multicolumn{2}{c}{$\begin{array}{c}\text { Half-Time } \\
\text { (No. renal units) }\end{array}$} \\
\cline { 3 - 4 } & & Positive & Negative \\
\hline At baseline* & Pos. & 31 & 6 \\
& Neg. & 7 & 10 \\
After saline and furosemide* & Pos. & 38 & 1 \\
& Neg. & 0 & 15 \\
\hline
\end{tabular}

* Sensitivity $82 \%$, specificity $63 \%$ and overall accuracy $76 \%$.

* Sensitivity $100 \%$, specificity $94 \%$ and overall accuracy $98 \%$.

Children were stratified into 2 groups according to age: 9 younger than 4 years (12 obstructed and 6 non-obstructed renal units) and 18 children 4 years old or older (26 obstructed and 10 nonobstructed renal units). The correlation between patient age and number of renal units with false results on both Doppler studies is shown in table 4. At baseline 6 of the 16 non-obstructed renal units (37.5\%) had falsepositive results $(r>0.70)$ and all were in children younger than age 4 years. After infusion of normal saline and furosemide, 1 renal unit $(6 \%)$ continued to have a resistive index of 0.70 , while in the remaining 5 resistive index decreased to less than 0.70 . At baseline 7 of 38 obstructed renal units $(18 \%)$ had false-negative results $(R I<0.70)$, including only 1 in a patient younger than age 4 years. After the addition of saline and furosemide resistive index increased to greater than 0.70 and none of the patients with obstruction continued to have a falsenegative result. It is evident that the addition of normal saline and furosemide decreases the number of false-positive results in children younger than age 4 years and false-negative results in older children.

Table 3. Comparison between the results of the 2 Doppler studies

\begin{tabular}{llll}
\hline \multicolumn{4}{c}{ No. Renal Units / Total No. } \\
& \multicolumn{2}{c}{$\%)$} & \\
& At baseline & $\begin{array}{c}\text { After saline \& } \\
\text { furosemide }\end{array}$ & $\begin{array}{c}\text { p Value (Fisher's } \\
\text { exact test) }\end{array}$ \\
\cline { 2 - 4 } Sensitivity & $31 / 38(81)$ & $38 / 38(100)$ & $<0.006$ \\
Specificity & $10 / 16(63)$ & $15 / 16(94)$ & $<0.04$ \\
Overall accuracy & $41 / 54(76)$ & $53 / 54(98)$ & $<0.0005$ \\
\hline
\end{tabular}


Table 4. Correlation between patient age and false results

\begin{tabular}{lccc}
\hline & \multicolumn{2}{c}{ Younger than 4 years } & \multirow{2}{*}{$\begin{array}{c}\text { 4 years or older } \\
\text { (obstructed) }\end{array}$} \\
\cline { 2 - 3 } & Nonobstructed & Obstructed & \\
\hline $\begin{array}{l}\text { No renal units } \\
\text { At baseline: }\end{array}$ & 6 & 12 & 26 \\
$\quad$ False pos. & 6 & - & - \\
$\quad$ False neg. & - & 1 & 6 \\
After saline and & & & \\
furosemide: & & - & - \\
$\quad$ False pos. & 1 & 0 & 0 \\
False neg. & - & & \\
\hline
\end{tabular}

*No lalse results in 10 nonobstructed renal units.
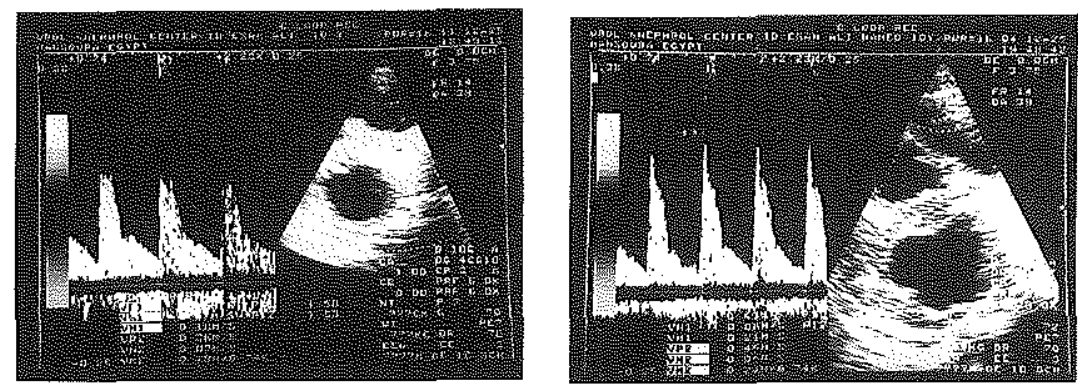

lig 2: Doppler ultrasound of obstructed kidney. A, at baseline (mean pulsatile index 1.5 , mean resistive index 0.81 ). B, after infusion of normal saline and administration of furosemide (mean pulsatile index 1.71, mean resistive index 0.88 ).
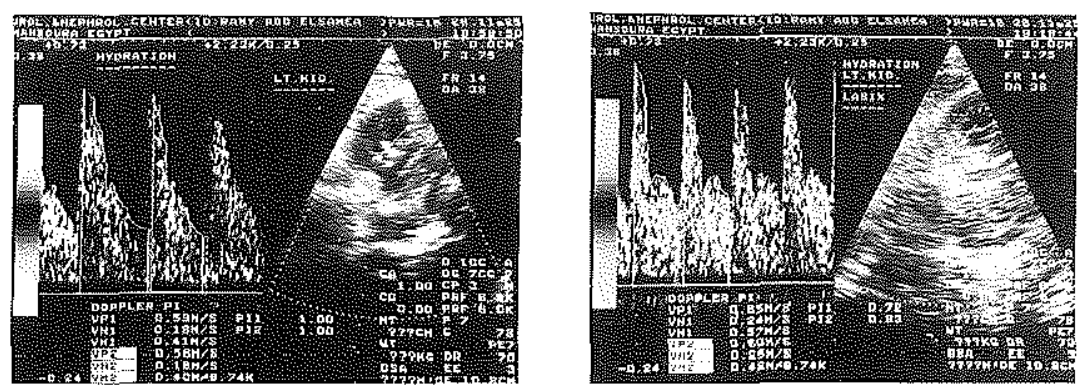

Fig. 3: Doppler ultrasound of normal kidney. A, at baseline ( mean pulsatile index 1.00 , mean resistive index 0.70 ). B. alter infusion of normal saline and administration of furosemide (mean pulsatile index 0.78 , mean resistive index 0.61 ). 


\section{Discussion}

Platt et al (1989a) recently described the use of renal resistive index to differentiate obstructive from non-obstructive pyelocaliectasis. In an effort to define a good discriminatory value to differentiate obstructive from non-obstructive dilatation they reported a large series of patients, including 70 with pyelocaliectasis (Platt et al., 1991). In the 38 obstructed kidneys mean resistive index $(0.77 \pm 0.05)$ was elevated compared to that of 32 with non-obstructive dilatation (mean $0.63 \pm$ 0.06 ). These 70 kidneys allowed Platt et al to plot a receiver operating curve that identified 0.70 as an optimal discriminatory resistive index value. This threshold value achieved $92 \%$ sensitivity, $88 \%$ specificity and $90 \%$ overall accuracy in diagnosing the presence or absence of obstruction in the adult population.

Few data exist regarding renal Doppler assessment of hydronephrotic kidneys in the pediatric population. It has been shown that the resistive index is frequently elevated above 0.70 in young children with normal upper urinary tracts (Gilbert et al., 1993; Wong et al., 1989; Bude et al., 1989; Keller et al., 1989). In an attempt to circumvent this age dependency, Keller et al (1991) recently evaluated the resistive index ratio (the ratio of resistive index of the potentially obstructed-to-contralateral normal kidney), achieving $77 \%$ sensitivity and $81 \%$ specificity. Also, Palmer et al (1991) and Keller et al (1992) recently investigated Doppler ultrasonography in children before and after intravenous furosemide administration, showing that furosemide causes the resistive index to increase above baseline in obstructed kidneys but it does not significantly affect the resistive index compared to baseline in normal and non-obstructed kidneys with pyelocaliectasis. In 1994 Bude et al demonstrated that infusion of normal saline and administration of furosemide significantly decrease the resistive index in non-obstructed renal units compared to baseline values. We recently confirmed the results of Bude et al in 14 children with normal upper urinary tracts (Shokeir et al., 1996b). In another experimental study we showed that infusion of saline and furosemide causes the resistive index to decrease in non-obstructed and increase in obstructed kidneys (Shokeir et al., 1997a). The same results were obtained in the present clinical study. The divergent responses of the obstructed and nonobstructed renal units to intravenous fluid load and furosemide have significantly improved the sensitivity, specificity and overall accuracy of the resistive index value of 0.70 as a predictor of renal obstruction.

Our results confirm those of previous studies that furosemide can cause a significant increase in the resistive index in obstructed renal units (Palmer et al., 1991; Keller et al., 1992). We agree with Bude et al (1994) that intravenous normal saline and furosemide decrease the resistive index in non-obstructed kidneys. However, other studies have 
shown that furosemide has no effect on the resistive index in nonobstructed kidneys in children (Palmer et al., 1991; Keller et al., 1992) as well as adults (Renwoden and Cochlin, 1992), although furosemide was given alone without an intravenous fluid load.

Our observation that the intravenous administration of normal saline and furosemide decreases the resistive index in non-obstructed kidneys is potentiated by the physiological fact that normal saline and furosemide decrease renal vascular resistance (Hook et al., 1966; Gerber et al., 1980; Ludens et al., 1968). Ludens et al (1968) demonstrated a significant dose related decrease in renal vascular resistance in dogs after intravenous infusion of nomal saline and furosemide. When the normal saline dose was maintained constant, larger doses of furosemide decreased vascular resistance. Similarly with a constant dose of furosemide, increased doses of saline decreased vascular resistance. It is likely that a similar decrease in renal vascular resistance is reflected in the decrease in renal resistive index in our series.

Notably all children in our series with false-positive results were younger than age 4 years. Similar results were obtained in our recent study of 28 non-obstructed renal units (Shokeir et al., 1996b). These results are in agreement with those of Bude et al (1994) who noted that the renal resistive index in healthy children is commonly elevated above the upper limit in adults $(0.70)$ in the first year of life and the overall trend shows a decrease with age. From age 4 years on the likelihood is low ( $2 \%$ probability) that the resistive index will be above 0.70 .

Presently diuretic renography is used at most centers as the gold standard for the diagnosis of obstruction. Kass et al (1985) evaluated 42 hydronephrotic kidneys with diuretic renography and pressure perfusion studies to diagnose or exclude obstruction. Both studies were highty accurate but a small number of false-positive and false-negative results were noted with each testing modality. An added benefit to radioisotope renography is the ability to measure differential glomerular filtration rate and arrive at a percent of total glomerular filtration rate contributed by each kidney. The calculation of half-time drainage is more objective in the diagnosis of obstruction. Kidneys able to excrete more than half of the radioisotope in less than 10 minutes with an empty bladder are considered non-obstructed and those requiring more than 20 minutes are considered obstructed. Although diuretic renography is non-invasive, it has the disadvantages of being expensive, using ionizing radiation and having a 10 to $15 \%$ rate of false-positive and indeterminate results. (Howman - Giles et al., 1987) 


\section{Conclusions}

The non-invasive nature of measuring renal resistive index gives it considerable appeal in its potential application to children with obstructive uropathy. The addition of normal saline fluid load and furosemide has significantly improved the sensitivity, specificity and overall accuracy of Doppler ultrasonography in the diagnosis of renal obstruction in children, particularly those younger than 4 years. 
Chapter 8

Renal Doppler ultrasound in children with equivocal obstructive uropathy: effect of intravenous normal saline fluid load and frusemide

Ahmed A. Shokeir, Abraham P. Provoost, Mohamed El-Azab, M. Dawaba, M. A. Shokeir, and Rien J.M. Nijman

Urology \& Nephrology Center and Pediatric Department, Mansoura University, Mansoura-Egypt; and Departments of Pediatric Surgery and Pediatric Urology, University Hospital, Erasmus University, Rotterdam, The Netherlands.

Br. J. Urol. (1997) 80: 313-318 



\section{Abstract}

\section{Objective}

To study the effect of hyperhydration with normal saline and frusemide on the renal resistive index (RI) in children with equivocal obstructive uropathy.

\section{Methods}

Twelve children (24 kidneys) with unilateral or bilateral hydronephrosis underwent isotopic diuretic renography and Doppler ultrasonography. All children had equivocal obstruction of the hydronephrotic renal units with half-time drainage $T_{I / 2}$ values between $10-20$ min. Doppler studies were carried out at baseline and after the infusion of normal saline and frusemide.

Results

Of the 24 kidneys, five were normal and 19 were hydronephrotic; compared with normal kidneys, the hydronephrotic units had a significantly lower glomerular filtration rate (GFR) and longer $T_{1 / 2}$. At baseline, the mean RI values of normal and hydronephrotic kidneys were not significantly different $(0.70$ SD 0.03 and 0.71 SD 0.04 , respectively). After the infusion of saline and frusemide, the mean $\mathrm{RI}$ of hyrdonephrotic kidneys $(0.67 \mathrm{SD} 0.07)$ was significantly $(\mathrm{P}=0.01)$ higher than that of normal kidneys $(0.60 \mathrm{SD} 0.02)$, but the response of $\mathrm{RI}$ in hydronephrotic kidneys was variable. Based on the $\mathrm{Rl}$ at baseline and after infusion, hydronephrotic kidneys could be categorized into three groups. Group $1(n=6)$ had an RI $<0.7$ before and after infusion, group $2(n=6)$ had a baseline RI $>0.7$ and $<0.7$ after infusion, and in groups $3(n=7)$ both Rls were $>0.7$. Kidneys in group 3 had the lowest GFR and the highest $T_{1 / 2}$ values. Five of these seven hydronephrotic kidneys eventually had deteriorating GFRs requiring surgical correction; the GFR of the remaining hydronephrotic kidneys remained stable.

\section{C'onclusions}

In children with equivocal obstructive uropathy based on diuretic renography, the determination of RI before and after infusion of normal saline and frusemide could be helpful in distinguishing obstructed from non-obstructed renal units. 


\section{Introduction}

The diagnosis of obstructive uropathy in children is a difficult and perplexing problem. Diuretic renography is the most widely accepted non-invasive procedure for the diagnosis of obstructive pyelocalyectasis (O'Reilly et al., 1978; Koff et al., 1980; Kass et al., 1985). In 1985 Kass and associates used the half-time drainage $T_{1 / 2}$ as an objective tool in the diagnosis of obstruction. $T_{1 / 2}$ is defined as the interval necessary for half of the isotope to be eliminated after administration of frusemide. Kidneys with a $T_{1 / 2} \leq 10 \mathrm{~min}$ are considered to be unobstructed and those with $\mathrm{T}_{1 / 2} \geq 20$ minutes are obstructed. Unfortunately, $15-20 \%$ of dilated collecting systems have indeterminate results, with a $T_{1 / 2}$ of 10-20 min (Kass et al., 1985). In this particular group of patients, diuretic renography must be augmented with other diagnostic procedures to determine whether the dilated collecting system is obstructed or not.

In 1989, Platt et al. described the use of Doppler ultrasonography to differentiate obstructive from non-obstructive pyelocalyectasis. The non-invasive measurement of the resistive index (RI) is particularly attractive for use in children with obstructive uropathy. We have recently shown that the sensitivity, specificity and overall accuracy of RI in identifying children with unequivocal obstructive uropathy $\left(T_{1 / 2}\right.$ $>20$ minutes) increased significantly with the addition of intravenous normal saline fluid load and frusemide (Shokeir et al., 1996c). In the present study, we determined the RI before and after infusion of normal saline and frusemide to identify obstruction in children in whom the diuretic renegraphy was equivocal $\left(\mathrm{T}_{1 / 2}\right.$ of $\left.10-20 \mathrm{~min}\right)$.

\section{Patients and methods}

The intrarenal haemodynamics in 12 children (seven boys and: five girls, mean age 4.3 years, SD 2.7, range 1-9; 24 kidneys) with unilateral or bilateral equivocal obstructive uropathy were evaluated using Doppler ultrasonography: and diuretic renography in separate sessions. The study group was highly selected so that one or both kidneys had pyelocalyectasis with a $T_{1 / 2}$ value between 10 and 20 min. Pyelocalyectasis was diagnosed by ultrasonography and excretory urography; in all patients, the hydronephrosis was discovered during childhood and was bilateral in seven and unilateral in five. The causes of pyelocalyectasis were pelviureteric junction narrowing in 11 and non-refluxing megaureter in eight renal units. In all the five patients with unilateral pyelocalyectasis the contralateral kidney was normal, with $T_{1 / 2}$ value $<10 \mathrm{~min}$. 
After the renographic and ultrasonographic studies, children were monitored with renography every 3 months during a follow-up period of 3 to 12 months. When a progressive decrease in GFR occured, the hydronephrotic units were surgically corrected.

\section{Doppler ullrasonography}

All children were well hydrated before ultrasonography and indwelling catheters were placed to ensure there were no bladder pressure artifacts. In every patient, Doppler ultrasonography was carried out at baseline and then repeated after the intravenous infusion of normal saline $(15 \mathrm{~mL} / \mathrm{kg})$ given during 30-60 min followed by an injection with frusemide $(1 \mathrm{mg} / \mathrm{kg}$, maximum $40 \mathrm{mg}$ ); the study was repeated $10 \mathrm{~min}$ after the injection. The Infusion of normal saline was continued during the entire second Doppler study, to replace urine output.

Ultrasonography was performed on a Toshiba (SSA-270A) unit (Toshiba Corporation Medical Systems Division, Tokyo, Japan) at a transducer frequency of $3.75 \mathrm{MHz}$. Renal morphology was examined in longitudinal and transverse planes. At least five Doppler spectra were obtained from more than three regions in each kidney in every study. The RI was obtained using software, from the formula RI = (peak systolic velocity-end diastolic velocity) / peak systolic velocity. Values of RI used in statistical analyses were overall means of the mean measurements in each study in the individual kidney.

\section{Diuretic renography}

Diuretic renography was performed using a standard protocol for all patients; a urethral catheter was placed in each child, an intravenous line established and normal saline given $(15 \mathrm{~mL} / \mathrm{kg})$. The patient was placed supine on the scanning table and ${ }^{99}{ }^{\mathrm{m}} \mathrm{Tc}_{\mathrm{M}}-\mathrm{MAG}_{3}$ injected intravenously at a dose of $2.11 \mathrm{MBq} / \mathrm{kg}$ and a standard renal scan performed; after $20 \mathrm{~min}, 1 \mathrm{mg} / \mathrm{kg}$ frusemide was injected intravenously. Sequential images were acquired by a gamma camera computer system for $30 \mathrm{~min}$ and the computed data processed to generate a time activity curve, the GFR and the $T_{1 / 2}$; the technique of diuretic renography was described previously (Shokeir et al., 1994). 


\section{Results}

Of the 24 kidneys investigated, five were normal and 19 hydronephrotic; the GFR, $T_{1 / 2}$ and RI values are summarized in Table 1. Compared to the normal kidneys, the hydronephrotic renal units had significantly lower GFR and longer $T_{1 / 2}$ values. At baseline, the mean (SD) Rl values were $0.70(0.02)$ and $0.71(0.04)$ for normal and hydronephrotic kidneys, respectively; the difference was not significant. After the infusion of saline and frusemide, the difference between RI of normal and hydronephrotic kidneys became significant ( $p<0.01$; Table 1). Compared with baseline values, the infusion of saline and frusemide caused a significant decrease in the mean RI of normal renal units ( $p<$ 0.001 ) and no significant change in the mean RI of hydronephrotic kidneys ( $p>0.05$; Table 1). However, the response of the hydronephrotic kidneys was remarkedly diverse. Representative Doppler waveforms in both conditions are given in Fig. 1 .

Table 1. Summary of results obtained from hydronephrotic and normal kidneys

\begin{tabular}{lccc}
\hline & $\begin{array}{c}\text { Normal } \\
\mathrm{n}=5\end{array}$ & $\begin{array}{c}\text { Hydro } \\
\text { nephrotic } \\
\mathrm{n}=19\end{array}$ & P* \\
\hline GFR (mL/min) & $52(2)$ & $46(9)$ & 0.01 \\
T1/2 (min) & $5(2)$ & $14(3)$ & $<0.0001$ \\
RI & & & \\
Basal & $0.70(0.02)$ & $0.71(0.04)$ & 0.7 \\
After saline+frusemide & $0.60(0.02)$ & $0.67(0.07)$ & 0.01 \\
\hline * Student $/$-test & & &
\end{tabular}

There was a strong positive correlation between the $T_{1 / 2}$ and $\mathrm{RI}$ of all kidneys after the infusion of normal saline and frusemide $(r=0.75$ $\mathrm{P}<0.01$; Fig. 2) but there was no significant correlation between $T_{1 / 2}$ and $\mathrm{RI}$ at baseline. Moreover, there was an inverse correlation between the GFR and RI of all kidneys in both conditions during the Doppler studies(Fig. 3).

Children were classified into two groups according to their age i. e. those aged $<4$ years (seven children with 14 kidneys, three normal and 11 hydronephrotic) and those aged $>4$ years (five children with 10 kidneys, two normal and eight hydronephrotic). There was no significant difference between the mean values of RI in children of both age groups in both conditions of Doppler studies. Of the normal kidneys, all three with an $\mathrm{RI}>0.70$ were in children younger than 4 years. 

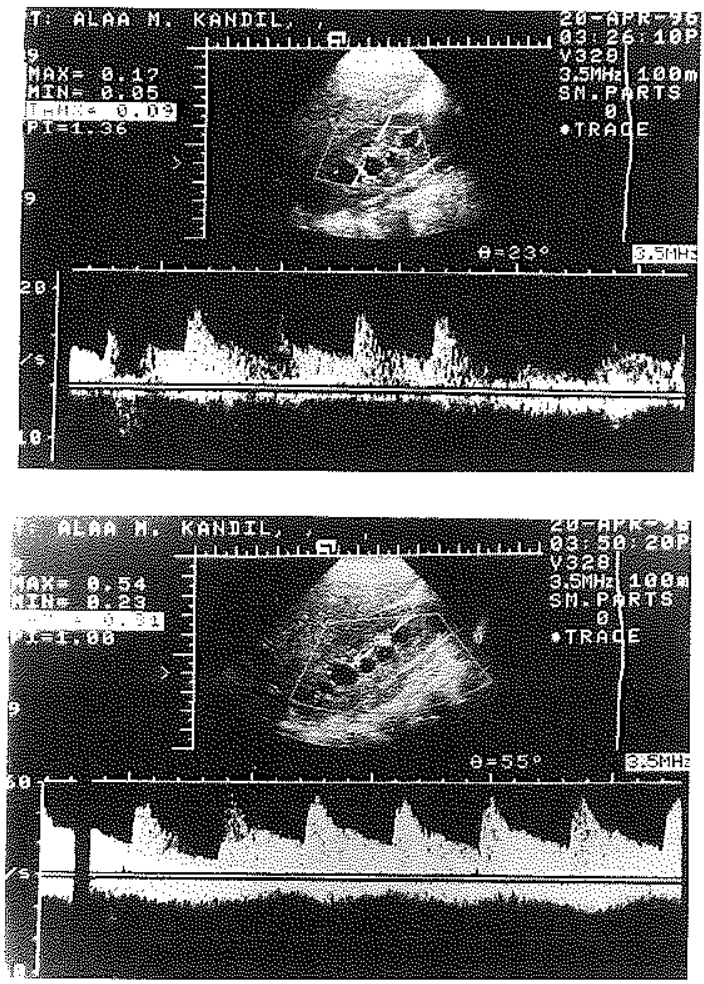

Figure 1: Doppler utlrasonography of a kidney with mild pyelocalyectasis: a) at basal condition ( $(\mathrm{l} \mathrm{l}=0.7 \mathrm{l})$, and $\mathrm{b}$ ) after infusion of normal saline and frusemide $(\mathrm{RI}=0.57)$.

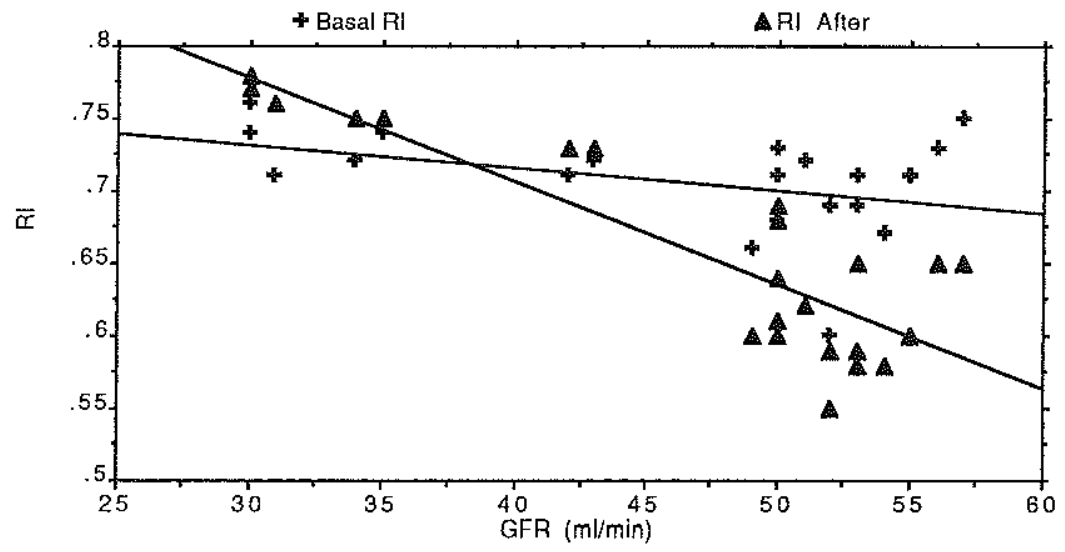

Figure 2: Correlation between $T_{1 / 2}$ and $R l$ at basal condition $(r=0.33, p=0.12)$ and after infusion of saline and fruscmide $(r=0.76, p=0.0001)$. 


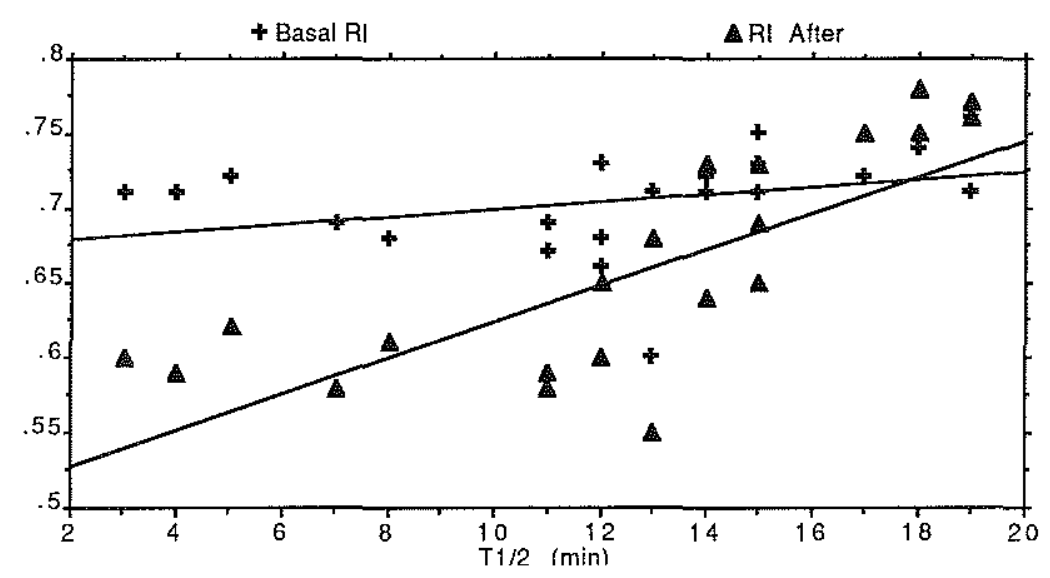

ligure 3: Correlation between GFR and RI at baseline $(r=-0.41, p=0.048)$ and after infusion of saline and fritsemide $(r=0.86, p=0.0001)$.

The 19 hydronephrotic kidneys were further classified into three groups according to the value of RI before and after the infusion of normal saline and frusemide. In group $1(n=6)$, RI was $<0.70$ at both times, in group $2(n=6)$ the basal RI was $>0.70$ and after infusion was $<0.70$, and in group $3(n=7)$ the RI was $>0.70$ on both occasions. The mean values of RI before and after infusion, the GFR, and $T_{1 / 2}$ of the three groups of hydronephrotic and the normal kidneys are summarized in Table 2. Compared with the normal kidneys and groups 1 and 2 , the kidneys in group 3 had the lowest GFR and the highest $T_{1 / 2}$ values. Furthermore, the clianges in $\mathrm{Rl}$ after the infusion of saline and frusemide were significantly different in group 3 and increased rather than decreased. Thus, the kidneys of group 3 showed all the characteristics compatible with obstruction of the hydronephrotic kidneys (Shokeir et al., 1996c).

All patients with hydronephrotic kidneys were followed using renography every 3 months; five of the seven kidneys of group 3 showed a progressive decrease in GFR and were surgically corrected.

Comparing the results of these five kidneys before and after surgical correction showed significant decrease in mean (SD) RI from $0.73(0.02)$ to $0.66(0.03)(P<0.01)$, and significant decrease in $T_{1 / 2}$ from $18.2(0.8)$ to $7.0(1.6)(\mathrm{P}<0.001)$ and no significant change of GFR. The hydronephrotic kidneys of groups 1 and 2 showed a stable GFR on surveillance and were not subjected to surgery. 
Table 2. Classification of kidneys according to the RI before and after infusion of normal saline and frusemide

\begin{tabular}{|c|c|c|c|c|c|}
\hline & \multirow[b]{2}{*}{ Normal } & \multicolumn{4}{|c|}{ Hydronephrotic group ${ }^{*}$} \\
\hline & & $\mathrm{I}$ & 2 & 3 & $p^{*}$ \\
\hline Number & 5 & 6 & 6 & 7 & \\
\hline Mean ( SD ) & & & & & \\
\hline RI baseline & $0.70(0.01)$ & $0.66(0.03)$ & $0.72(0.01)$ & $0.73(0.02)$ & $>0.05$ \\
\hline $\begin{array}{l}\text { Ri alter } \\
\text { infusion }\end{array}$ & $0.60(0.01)$ & $0.58(0.02)$ & $0.66(0.02)$ & $0.76(0.02)$ & $<0.05$ \\
\hline$\Delta \mathrm{RI}$ & $-0.10(0.02)$ & $-0.08(0.02)$ & $-0.06(0.02)$ & $0.02(0.01)$ & $<0.05$ \\
\hline $\begin{array}{l}\mathrm{GFR} \\
(\mathrm{mL} / \mathrm{min})\end{array}$ & $52(1.7)$ & $52(1.9)$ & $53(3)$ & $35(5)$ & $<0.05$ \\
\hline $\mathrm{T}_{1 / 2(\mathrm{~min})}$ & $5.4(1.9)$ & $12(0.7)$ & $14(1.2)$ & $17(1.8)$ & $<0.05$ \\
\hline
\end{tabular}

\section{Discussion}

The differentiation of true renal obstruction from non-obstructive dilatation in children is difficult. Grey-scale ultrasonography is generally of little value in this clinically important distinction. Standard excretory urography, even with diuretic augmentation, does not permit the objective diagnosis or exclusion of urinary obstruction. The Whitaker test has not achieved extensive popularity because it has potential risks and is invasive (Whitaker, 1973; Jaffe and Middleton, 1980). As a substitute, there is considerable interest in the diuretic renal scan, which provides an accurate measurement of individual renal function, is not invasive and is tolerated well by patients( $\mathrm{O}^{\prime}$ Reilly et al., 1978; Koff et al., 1980; Kass et al., 1985). However, the rate of washout of the radionuclide is influenced by several factors, including renal functions, ability to respond to the diuretic, distensibility and the volume of the collecting system (Kass et al., 1985). Therefore, 15-20\% of children with pyelocalyectasis have indeterminate results from diuretic renography (Kass et al., 1985). Platt et al. (1989a) recently described the use of the renal RI to differentiate obstructive from nonobstructive pyelocalyectasis. In an effort to define a good discriminatory value to differentiate obstructive from non-obstructive dilatation, they reported on a large series of patients, including 70 with pyelocalyectasis. In the 38 obstructed kidneys, the mean (SD) RI of $0.77(0.05)$ was greater than the 32 kidneys that had non-obstructive dilatation (0.63 SD 0.06). The results from these 70 kidneys were used to construct a receiver-operating curve that identified an RI of 0.70 to be the optimal discriminatory value. This threshold value gave a sensitivity of $92 \%$, a specificity of $88 \%$, and an overall accuracy of $90 \%$ in 
diagnosing the presence or absence of obstruction in the adult population (Platt et al., 1989b).

There is little information about the renal Doppler assessment of hydronephrotic kidneys in children, it has been shown that the RI frequently exceeds 0.70 in young children with normal upper urinary tracts (Gilbert et al., 1993; Wong et al., 1989; Bude et al., 1992). In an attempt to circumvent this relationship with age, Keller et al. (1991) examined the RI ratio (the ratio of the RI in the potentially obstructed kidney to the contralateral normal kidney), achieving a sensitivity of $77 \%$ and a specificity of $81 \%$. Additionally, Palmer et al. (1991) and Keller et al. (1992) investigated Doppler ultrasonography in children before and after intravenous frusemide, showing that frusemide causes the RI to increase above baseline in obstructed kidneys and did not significantly affect the RI in normal and unobstructed pyelocalyectatic kidneys. Bude et al. (1994) showed that the infusion of normal saline and administration of frusemide significantly decreased the RI of unobstructed kidneys compared with baseline values; we recently confirmed these results in 14 children with normal upper urinary tracts (Shokeir et al., 1996b). In another clinical study we showed that hyperhydration with normal saline and the administration of frusemide significantly increased the sensitivity, specificity and overall accuracy of Doppler ultrasonography in the identification of obstruction in 27 children with unilateral or bilateral hydronephrosis (Shokeir et al., 1996c). In that, all the hydronephrotic kidneys had unequivocal obstruction with a $T_{1 / 2}>20 \mathrm{~min}$. In the present study, intravenous normal saline and frusemide also helped to differentiate between normal and equivocally obstructed kidneys with a $T_{1 / 2}$ of 10-20 min. Moreover, a significant number of normal and hydronephrotic kidneys with an Rl $>0.70$ at baseline had an RI of $<0.70$ after the infusion of saline and frusemide. Thus hyperhydration and diuresis increase the specificity of an $\mathrm{RI}$ of 0.70 in discriminating obstruction, by reducing the number of false-positive results.

The finding that the intravenous administration of normal saline and frusemide can decrease the RI in unobstructed kidneys is supported by the physiological effect of both normal saline and frusemide in decreasing renal vascular resistance (Hook et al., 1966; Gerber and Nies 1980; Ludens et al., 1968). Ludens et al. (1968) reported a significant decrease in renal vascular resistance in dogs after this treatment, which was also dose related; when the dose of normal saline was maintained constant, larger doses of frusemide decreased the vascular resistance. Similarly, with a constant dose of frusemide, increased doses of saline decreased the vascular resistance. It is likely that a similar decrease in renal vascular resistance is reflected in the decrease of renal RI in the present series. 
When equivocal obstruction is diagnosed by renography $\left(\mathrm{T}_{1 / 2}\right.$ $10-20 \mathrm{~min})$, Doppler ultrasonography may be of potential value in distinguishing between obstructed and unobstructed kidneys. Initially, all hydronephrotic kidneys in the present series were managed by conservative surveillance, with follow-up renography to detect changes in GFR indicating a deterioration in renal function. Based on RI before and after infusion, groups of hydronephrotic kidneys could be distinguished; those with both Rls $>0.70$, had a high risk of renal impaiment. Five of seven of these kidneys showed deterioration of GFR and were surgically repaired. In a previous study, Rls $>0.70$ before and after saline and frusemide indicated obstruction in kidneys with a $T_{1 / 2}>20 \mathrm{~min}$; apparently, the same applies for kidneys with slightly lower $T_{1 / 2}$ values. In the five kidneys that were surgically corrected, $\mathrm{T}_{1 / 2}$ was 17-19 min, while those with a stable GFR had $\mathrm{T}_{1 / 2}$ values of $11-15 \mathrm{~min}$. Thus it appears that a combination of a $T_{1 / 2}>15$ min and an RI of $>0.70$ after saline and furesmide is characteristic of the presence of obstruction in a hydronephrotic kidney.

The RI correlated closely with $T_{1 / 2}$ in children with equivocal pyelocalyectasis after the infusion of saline and frusemide; thus the RI is a useful variable in the evaluation of hydronephrosis, even in its early and mild phases. These results agree with those obtained by Gilbert et al. (1993) who reported a close correlation between the findings of renal Doppler ultrasonography and drainage values obtained with renal scintigraphy / diuretic renography in 28 hydronephrotic paediatric kidneys. However, Chen et al. (1993) stated that not all renal obstruction produces a significantly elevated RI; in mild obstruction there may be low and normal RI values.

The renal Doppler examination provides physiological information and is insufficient for precise anatomical definition. Therefore, it cannot be used as a single method of evaluation in a patient with hydronephrosis. Thus, the value of using the RI is to provide corroboration of the initial diuretic renogram and as a noninvasive modality for monitoring the dilated collecting system under observation. Thus, a hydronephrotic kidney with an un-obstructive or equivocal $T_{1 / 2}$ on diuretic renography can be followed closely with renal Doppler ultrasonography, obviating the need for frequent isotopic renal scintigraphy. Additionally, it may be a useful non-invasive method for monitoring patients after re-constructive surgery of the upper urinary tract. 

Chapter 9

General discussion 



\section{Introduction}

The diagnosis of urinary tract obstruction is a difficult problem particularly in children. Several methods have been used to identify obstructive uropathy, but none of them is satisfactory. The potential usefulness of resistive index (RI) obtained during Doppler ultrasonography (DU) was recently described by Platt et al (1989a). The noninvasive nature of the measurement of renal RI gives it considerable appeal in its potential application to patients with obstructive uropathy. The present thesis deals with the role of RI in the diagnosis of obstructive uropathy.

\section{RI in obstructed and non-obstructed kidneys.}

Renal obstruction like other states of increased vascular resistance, produces a change in the Doppler waveform causing an increase in RI. An RI value of 0.70 is accepted by most investigators to be the upper limit of normal in the adult population. However, renal outflow obstruction is not the only cause of an elevation of RI. The RI value could exceed 0.70 without obstruction in children, particularly infants, and in patients with non-urological renal diseases. Moreover, dehydration, hypotension and a low heart rate appear able to cause an elevation of RI without a true change in renal vascular impedance. However, a normal RI in these conditions is still a strong argument against obstruction. In the first clinical study of this thesis (chapter 6) we have observed that the RI is $\geq 0.70$ in $54 \%$ of non-obstructed kidneys in fasting children. After hydration, the RI regains its normal value; this observation addresses the importance of at least oral hydration for the proper interpretation of Doppler studies.

\section{RI in mild obstruction}

Most investigators agree that marked partial ureteric obstruction results in an increase in intra-renal vascular resistance that is reflected as an elevation of RI. The increase of vascular resistance in mild obstruction is still a matter of controversy and has generated a heated debate. In the second experimental (chapter 4) and third clinical (chapter 8) studies of this thesis we show that RI is a helpful diagnostic modality even in mild and equivocal obstruction. 


\section{Diagnostic accuracy of $R I$}

In the first experimental study of this thesis (chapter 3), we demonstrated that infusion of saline and furosemide causes the RI to decrease in non-obstructed kidneys and to increase in obstructed ones. This divergent response helps identify obstruction and is better than a simple discriminatory value of RI. We used this principle in the second clinical study of the thesis (chapter 7) in 54 hydronephrotic paediatric. kidneys and showed that infusion of normal saline and administration of furosemide significantly increased the sensitivity, specificity and overall accuracy of RI in the diagnosis of renal obstruction .

\section{RI and prediction of recoverability of renal function}

In the last experimental study of this thesis (chapter 5), we showed that the recovery of renal function could not be predicted from the changes of RI before de-obstruction. However, we noted that a reversal of a previously elevated RI could be used as an early indicator that recovery of renal function is likely. This could be applied clinically by monitoring RI before and after the temporary release of obstruction via a percutaneous rephrostomy. In the future, we may be able to couple DU with the administration of vasoactive drugs to detect a reversal of a high RI to predict recovery following corrective surgery.

\section{Relation of RI with diuretic renography}

In the present thesis, we supported the recent urological and radiological reports that there is a good positive correlation between RI and half-time drainage $\left(T_{1 / 2}\right)$. Thus, a hydrorephotic kidney with a nonobstructive $T_{1 / 2}$ on diuretic renography can be followed closely with surveillance renal DU, obviating the need for frequent isotope renal scintigraphy. 


\section{References}

Algood CB, Narender S, Fairvhild T, Mayo ME 1983 Experimental study of ureteral calculus disease: cffects of calculus size, obstruction and hydration. I Urol 130: 9991004

Arina M, lshibaski M, Usami M, Sagawa S, Mezutoni S, Sonoda T, Ichikawa S, llara H Nagano S 1979 Analysis of the arterial blood flow patterns of normal and allografted kidneys by the directional ultrasonic Doppler technique. J Urol, 122:587-591

Bassiouny IE 1992 Salvage pyeloplasty in non-visualizing hydronephrotic kidney secondary to ureteropelvic junction obstruction. J Urol 148: 685-687

Belis IA, Belis TE, Lai JCW, Goodwin CA, Gabriele OF 1982 Radionuclide determination of the individual kidney function in the treatment of chronic renal obstruction. IU Urol 127: 636-641

Boyarsky S, Martinez J 1964 Pathoplysiology of the ureter: partial ligation of the ureter in dogs. Invest Urol 2: 173-180

Bude RO, DiPietro MA, Platt JF, Rubin JM 1994 Effect of furosemide and intravenous normal saline fluid load upon the renal resistive index in nonobstructed kidneys in children. J Urol I51:438-441

Bude RO, DiPietro MA, Plalt JF, Rubin JM, Miesowicz S, Lundquisı C 1992 Age dependency of the renal resistive index in healthy children. Radiology $184: 469-473$

Carr MC, Peter CA, Retik AB, Mandell J 1994 Urinary levels of the renal tubular enzymes $\mathrm{N}$ - acetyl- B- glucosaninidase in unilateral obstructive uropathy. J Urol 151 : $442-445$

Chen JH, Pu YS, Liu SP Chiu TY 1993 Renal hemodynamics in patients with obstructive uropatlyy evaluated by duplex Doppler sonography. J Urol 150: 18-21

Chevalier RL, Jones CE 1986 Contribution of endogenous vasoactive compound to renal vascular resistance in neonatal chronic partial ureteral obstruction. J Urol 136:532-535

Chibber PJ, Chisholm GD, Hargreave TB, Merrick MV 1981 99m Technetiun DMSA and the prediction of recovery in obstructive uropathy. $\mathrm{Br} J$ Urol, 53: 492-495

Claesson G, Josephson S, Robertson B 1983 Experimental partial ureteric obstruction in newborn rats. IV. Do the morphological effects progress continuously. J Urol 130: 12171222

Cronan JJ, Tublin ME 1995 Role of the resistive index in the evaluation of acute renal obstuction. A.rR 164: 377-378

Cronan JJ 1991 Contemporary concepts in imaging urinary tract obstruction. Radiol Clin North Am 29: 527-542

Dejter SW Jr, Eggli DF, Gibbons MD 1988 Delayed management of neonatal hydronephrosis. J Urol 140: 1305-1309 
Deluca FG, Swenson On Smythm B 1961 The effects of chronic mechanical obstruction on ureteral peristalsis. J Urol 85:497-501

Dtilion HK 1998 Prenatally diagnosed hydronephrosis: the Great Omond Street experience. Br J Urol 81, suppl 2: 39-44

Elder IS, Stansbrey R, Dabns BB, Selzman AA 1995 Renal histological changes secondary to ureteropelvic junction obstruction. I Urol 154: 719-722

El-Hammady S, Shokeir AA 1994 A novel technique of ureteroneocystostomy (extravesical seromuscular tunnel): an experimental study in dogs. 1. preliminary results. Urology, 45: 339-334

Gerber JG, Nies AS 1980 Furosenide induced vasodilation: importance of the state of hydration and filtration. Kid Int $18: 454-459$

Gilbert R, Garra B, Gibbons MD 1993 Renal diptex Doppler ultrasound: an adjunct in the evaluation of hydroneplrosis in the child. J Urol I50:1192-1194

Gillenwater IY, Westervelt FB Jr, Vaughan ED Jr, Howards SS 1975 Renal function after release of chronic unilateral hydronephrosis in man. Kid Int, 7:179-186

Gillenwater JY: The pathophysiology of urinary obstruction. In Campbell's Urology 5th edition, Walsh, Gittes, Perlumutter, Stamey (cds). Saunders company. Chap 11. pp 499532,1992 .

Goullieb RH, Luhmanu K, Oates RP 1989 Duplex ultrasound evaluation of nomal native kidneys and native kidneys with urinary tract obstruction. J Ultrsound Med 8: 609-61 I

Gregoir W, Van Regemorter GV 1964 Le retlux vesicourcteral congenital. Urol Int. 18: 122

Guze LB, and O'shea W 1958 Experimental hydronephrosis produced by irradiation of the ureter. I Urol 79:801-806

Hinman $F 1943$ The condition of renal cotnterbalance and the theory of renal atrophy of disuse. I Urol 49: 392-400

Holmund D, Hassier O $1965 \wedge$ method for studying the ureteral reaction to arlijicial concrements. Acta Chir Scand 130: 335

Iook JB, Blatt AH, Brody M.l, Williamson HE 1966 Effects of several salturetic-diuretic agents on renal hemodynamics. J Pharmacol Exp Ther $154: 667-673$

Ilowman - Gilcs R, Uren R, Roy LP, Filmer RB 1987 Volume expansion diuretic renal scan in urinary tract obstruction. I Nucl Med $28: 824-829$

Huland II, Gonnermann D, Werner B, Possin U 1988 A new test to predict reversibility of hydroncphrotic atrophy after stable partial unilateral ureteral obstruction. I Urol I40: 1591* 1594 
Hvid-Jacobson K, Thonsen HS, Niclscn SL 1990. Dinresis renography: $A$ simultaneous comparison between ${ }^{13 !}$ I-hippuran and ${ }^{5 / 16} \mathrm{Tc}-\mathrm{MAG}_{3}$. Acta Radio! 31: 83-86

Ichikawa I, Brenner BM 1979 Local intrarenal vasoconstrictor-vasodilator interactions in mild partial ureteral obstruction. Amer J Physiol 236 : F 131- F 140

Jaffe RB, Middleton AW 1980 Whitaker test: Differentiation of obstructive from nonobstructive uropathy. AJR 134: 9-15

Johnston JH, Evans IP, Glassberg KI, Shapiro SR: Pelvic hydroneplurosis in children: a review of 219 personal cases. I Urol 117: 97-101, 1977.

Josephson S, Aperia A, Lannergren K, Wikstad I 1987 Partial ureteric obstruction tin the pubescent rat. 1. Long-term effects on renal function. I Urol [38: 414-4]8,

Joseplason S 1983 Experimental obstructive hydronephrosis in newborn rats. IIl. Long-term effects on renal function. 3 Urol 129: 396-400

Kalika V. Bard RH, lloreta A. Freeman LM, HellersS, Blaufox MD 1981 Prediction of renal functional recovery after relief of upper urinary tract obstruction. I Urol, 126: 301305

Kass E.J. Majd M, Belman AB 1985 Comparison of pediatric renogram and the pressure perfusion study in children. J Urol $134: 92-96$

Keller MS 1989 Renal Doppler sonography in infants and children. Radiology 172 : 603604

Keller MS, Gracia CI, Krosvik H, Weiss RM, Rosenfield NS 1991 Resistive index ratios in the US differentiation of unilateral obstructive Vs. non-obstructive hydronephrosis in children. Ped Rad 21 : 426, abstract 1

Keller MS, Krosvik HE, Weiss RM 1992 Diuretic Doppler sonography, with correlative scintigraphy in children with hydronephrosis. Abstract from Society of Pediatric Radiology. P. 49, May 14-17

Kerr WS Ir 1954. Elfect of complete ureteral obstruction for onc week on kidncy function. I Appl Physiol 6: 762

Kerr WS Ir 1956 leffects of complete ureteral obstruction in dogs on kidney function. Am I Plhysiol 184: 521-526

Kim SH, Kim WH. Choi BI, et al 1990 Duplex sonography of the native kidney. Resistive index Vs serum creatinine. 1 Ultrasound Med 9: S1-S124

Koll SA, Campbell KD 1994. The non-operative management of unilateral neonatal hydronephrosis: natural history of poorly functioning kidneys. J Urol 152: 593-595

Koff SA. Thrall JH. Keyes IW 1980 Assessment of hydroureteronephrosis in children using diusetic radionuclide urography. J Urol $123: 531-534$ 
Krueger RP, Ash JM, Silver MM, Kass EI, Gilmour RF, Alton DI, Gilday DL, Churchill BM 1980 Primary hydronephrosis. Assessment of diuretic renography, pelvis perlusion pressure, operative fundings, and renal and ureteral histology. Urol Clin North Am 7: 231242

Lich R .Jr, Howerton LW, Davis LA 1961 Recurrent urosepsis in children. J Urol 86: 554558

Lome LG, Pinsky S, Levy L 1979 Dynamic renal scan in the non-visualizing kidney. .J Urol 121: $148-152$

Ludens JH, Hook JB, Brody MJ, Williamson HE 1968 Enhancement of renal blood flow by furosemide. J Pharmacol Exp Ther $163: 456-460$

Marberger M, Altwein JE, Straub E, Wulff HD, Hohenfeliner R 1978 The Lich-Gregoir antireflux Plasty: experience with 371 children. J Urol 120: 216-219

McDougal WS, Flanigan RC 1981 Renal finctional recovery of the hydronephrotic kidncy predicted before relief of the obstruction. lnvest Urol, 18:440-442

Mitchell TS, Lee S, Gittes RF 1974 Hypotrophy of hyrertrophied kidneys in response to renal transplantation in rats. lnvest Urol $12: 213-217$

Mjalmas K, Lobr G, Tamminen-Mobius T, Seppanen .J, Olbing H, Wikstrom S 1992 Surgical results in the international reflux study in children (Europe). J Urol 148: 16571661

Mostbeck GH, Gossinger HD. Mallek R. et al 1990 Effect of heart rate on Doppler measurments of resistive index in renal arteries. Radiology 175; 511-513

Muller-Suur R, Bois-Svensson I, Mesko L 1990 A comparative study of renal scintigraphy and clearance with technetium-99m-MAG3 and iodine-I23-hippuran in patients with renal disorders. I Nucl Med 31: 181 [-1817

Norris CS, Pfeiffer IS, Rittgers SE, et al 1984 Noninvasive evaluation of renal artery stenosis and renovascular resistance. J Vasc Surg 1:192-201

Ordorica RC, Lindfors KK, Palmer IM 1993 Diuretic Doppler sonography following successful repair of renal obstruction in children. J Urol 150:774-777

O'Reilly PH, Testa HJ, Lawson RS, Farrar DJ, Edwards EC 1978 Diuresis renography in equivocal urinary tract obstruction. Br J Urol $50: 76-80$

Palmer IM, Lindfors KK, Ordorica RC, Marder DM 1991 Diuretic Doppler sonography in postratal hydronephrosis. I Urol, 146: 605-608

Patriquin JIB, O'Regan S, Robitaille P 1989 Hacmolytic - uremic syndrome: lntrarenal arterial Doppler patterns as a useful guide to therapy. Radiology 172: 625-628

Pavia J, Ros D, Piera C, Pavia A, Bassa P. Giralt E, Setoain J 199] Comparative study of

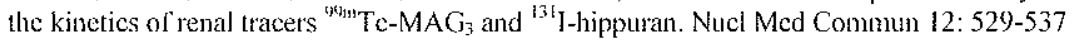


Platt IF 1992 Duplex Doppler evaluation of native kidney dysfunction : obstructive and non-obstructive disease. AIR 158: [035-1042

Platt JF, Ellis JH, Rubin JM 1991 Examination of native kidneys with Duplex Doppler ultrasound. Seminars in Ultrasound, CT, and MR, $12: 308-318$

Platt IF, Ellis JH, Rubin JM 1995 Role of renal Doppler imaging in the evaluation of acute renal obstruction. A.R 164: 379-380

Platt JF, Rubin JM, Ellis HM, DiPietro MA 1989a Duplex Doppler US of the kidney: differentiation of obstructive from nonobstructive dialatation. Radiology 17 : 515-517

Platt JF, Rubin IM, Ellis JH 1989b Distinction between obstructive and nonobstructive pyelocaliectasis with duplex Doppler sonography. AJR 153: 997 -I000

Provoost AP, De Keijzer MH, Wessel JN, Molenaar JC 1989 Renal function in singlekidney rats. Progr in Pediat Surg 23: 18-41

Provoost AP, Molenaar JC 1981 Renal function during and after a temporary complete unilateral ureter obstruction in rats. Invest Urol 18: 242-246

Provoost AP. Van Aken M, Molenaar IC 1990 Long term follow-up of renal function in rats with unilateral hydronephrosis. Scand J Urol Nephrol 24: 127-132

Provoost AP, Van Aken M, Molenaar JC 1991 Sequential renography and renal function in Brown-Norway rats with congenital hydronephrosis. I Urol 146: 588-591

Ransley PG, Dhillon HK, Gordon I, Duffy PG, Dillon MI, Barratt TM 1991 The postnatal management of hydronephrosis diagnosed by prenatal ultrasound. J Urol 144: 584-587

Renowden SA, Cochlin DL 1992, The effect of intravenous furosemide on the Doppler wave form in normal kidnsys. J Ultrasound Med 11: 65-68

Restaino IG, Kaplan BS, Ducker JW 1990 Hyperfiltration and the progression of renal injury. AUA Update series, Volume 9, Lesson 18 PP138-143

Ritkin MD, Needleman L, Pasto ME, Kurtz AB, Foy PM, McGlynn E, Canino C, Baltarowich OH. Penmell RG, Goldgerg BB 1987 Evaluation of renal transplant rejection by duplex Doppler examination: value of the resistive index. AJR 148: 759-762

Rodgers PH, Bates JA, Irving HC 1992 Intrarenal Doppler ultrasound studies in normal and acutely obstructed kidneys. Br J Rad 65: 207-212

Ryan PC, Fitzpatrick IM 1987 Partjal ureteral obstruction: a new variable canine experimental model. J Urol 137: 1034-1038

Ryan PC, Maher KP, Murphy B, Hurley GD, Fitzpatrick JM 1987 Experimental partial ureteric obstruction: pallsophysiological changes in upper tract pressures and renal blood flow. J Urol 138: 674-678 
Schelthout W, Simons M, Oosterlinck W, De Sy WA 1983 Evaluation of "\%mincdimercapto-succinic acid renal uptake as an index of individual kidney function after acute ureteral obstruction and de-obstruction: an experimental study in rats. Eur Urol 9: 221-226

Schimer HKA, Hendricks FB 1969 Metabolic aspects of the counterbalance theory in hydronephrosis. J Urol 101: 267-269

Seremetis GM, Maizels M 1996 TGF-b mRNA expression in the renal pelvis atter experimental and clinical treteropefvic obstruction. .J Urol 156: 261-266

Shapiro SR, Bennett AH 1976 Recovery of renal fimction after profonged unilateral ureteral obstruction. J Urol 1 I5: 136-\$40

Sherman RA, Blaufox MD 1980 Obstructive uropathy in patients with non-visualization on renal scan. Nephron 25: 82-86

Shokeir AA 1995 Partial ureteral obstruction: a new variable and reversible canine experimental model. Urology $45: 953-957$

Shokeir AA, El-Diasty TA, Gad HM, Sobh MA, El-Sherif AK, Gaber O, Ghoneim MA 1994 Radioisotopic evaluation of renal function in cyclosporine-treated pediatric and adults renal transplant recipients and their living donors. Transplantation 58:1171-1 175

Shokcir AA, El-Hammady S 1996 A novel technique of ureteroneocystostomy ( extravesical seromuscular tunnel): an experimental study in dogs, II. optimization of surgical technique. Urology, 48: 917-922

Shokeir AA, Nijman RJIM, EJ- Azab M, Provoost AP 1997a Partial ureteral obstruction: Effect of intravenous normal saline and furosemide upon the renal resistive index. I Urol, 157: 1074-1077

Shokeir AA, Nijman R.MM, El- Azab M, Provoost AP 1997b Partial ureteral obstruction: Role of resistive index in stages of obstruction and release. Urology 49: 528.535

Shokcir AA, Nijman RJM, El-Azab M, Porvoost AP 1996a Partial ureteal obstruction: a study of Doppler ultrasonography and diuretic renography in different grades and Jurations of obstruction. Br I Urol, 78: 829-835

Shokeir AA, Provoost AP, El- Azab M, Dawaba M, Nijman R.JM 1996b Renal Doppler ultrasound in childen with normal upper urinary tracts: Effect of fasting hydration with normal saline, and furoscmide administration. Urology 47: 740- 744

Shokeir AA, Provoost AP, El-Azab M, Dawaba M, Nijman RJM 1996e Renal Doppler ultrasound in children with obstructive uropathy: Effect of intravenous normal saline fluid load and furosemide. IJ Urol, 156:1455-1458

Stecker JF Jr, Gillenwater JY 1971 Experimental partial ureteral obstruction. I. Altcration in renal function. Invest Urol 8: $377-385$

Steinhardt GF, Ramon G, Salinas-Madrigal L: Glomerzloscierosis in obstuctive uropathy. I Urol 140: 1316-1318, 1988. 
Stenberg A, Olsen L, Josephson S, Robertson B, Persson AE 1985 Partial ureteric obstruction in weanling rats. I. L.ong-term effects on the renal morphology. Scand I Urol Nephrol 19: 139-146

Talarani (j, Farinelli R, Zavagli G, Logallo G, Farinelli A 1987 Tubule recovery after obstructive neplaropathy rclief: the value of enzymuria and microproteinuria. J Urol 138: 2427

Thurmher S, Kzika AA, Hricak H, Mattei P, Aboseif S, Eigelstad B, Price DC I989 Noncontrast and contrast enhaned $M R$ imaging in the evaluation of partial ureteral obstruclion: an experimental study in the micropig. Invest Rad 24: 544-554

Ulm AH, Miller F 1962 An operation to produce experimental reversible hydronephrosis in dogs. J Urot 88:337-341

Uirich JC, York IP, Koff SA 1995 The renal vascular response to actstely elevated intrapelvic pressure; resistive index measurement in experimental urinary obstructions. J Urol 154: 1202-1204

Vaughan ED Jr 1995 Commentary on the renal resistive index. I Urol 154:922

Valughan ED ir. Gillenwater IY I97] Recovery following complete chronic unilaterat ureteral occlusion: functional, radiographic and pathologic alterations. If Urol t06: 27 -35

Vaughan ED Ir, Sorenson EI, Gillenwater IY 1970 The renal hemodynamic response to chronic unilateral complete ureteral occlusion. J Urol $8: 78-90$

Walton G, Buttyan R, Garcia-Montes E, Olsson CA, Hensle TW, Sawczuk IS, 1992 Renal growth factor expression daring ealy phase of experimental hydronephrosis. J Urol 148: $510-514$.

Whitaker RH 1973 Methods of assessing obstruction in dilated ureters. Br J Urol 45: $15-22$

Wong SN. Lo RN, Yu EC 1989 Renal blood flow pattern by noninvasive Doppler ultrasound in normal children and acute renal failure patients. J Ultrasound Med 8 : 135141 



\section{SUMMARY}

We developed a new experimental canine model of partial ureteral obstruction that is simple, reliable, and reversible (chapter 2). We used this model to induce unilateral partial ureteral obstruction of different grades in three experimental studies.

In the first experimental study (chapter 3), we investigated the effect of intravenous normal saline fluid load, with and without furosemide, upon the renal resistive index (RI) of obstructed and nonobstructed kidneys. We found that in unilateral partial ureteral obstruction, addition of intravenous normal saline and furosemide cause the RI to increase in obstructed kidney and to decrease in non-obstructed kidney. Such a divergent response may be useful for the development of a pharmacologically challenged Doppler examination to diagnose better potentially obstructed kidneys.

In the second experimental study (chapter 4), we investigated the changes in renal RI and renal function with time during different grades (Chapter 4) of partial ureteric obstruction, and we determined the correlation between the ultrasonographic and renographic findings. We found that unilateral partial ureteric obstruction increased the RI and half-time drainage $\left(T_{1 / 2}\right)$ and decreased the effective renal plasma flow (ERPF) of the corresponding kidney, together with a decrease of RI and an increase in ERPF of the contralateral kidney. The more severe the obstruction, the greater the increase in $\mathrm{RI}$ and $\mathrm{T}_{1 / 2}$ and the decrease in ERPF. After the obstruction stabilized, RI and $T_{1 / 2}$ were positively correlated.

In the third experimental study (chapter 5), we investigated the changes in RI and renal function before and after release of different grades of partial unilateral ureteral obstructions. From that study we concluded that: 1) Unilateral partial ureteral obstruction produces an elevation of RI and $T_{1 / 2}$ and a fall in ERPF of the corresponding kidney. 2) After relief of obstruction, renal function is regained with associated reversal of RI. 3) Functional parameters ( $T_{1 / 2}$, Rl or ERPF) of the obstructed kidney do not predict the recovery of ERPF after release of obstruction, 4) Rapid reversal of a previously elevated RI is an early indicator of recoverability of renal function after relief of ureteral obstruction.

After the experimental studies, we carried out three clinical studies in children. In the first clinical study (chapter 6), we investigated the effect on the renal RI of fasting, intravenous infusion of normal saline, and administration of furosemide in children with normal upper urinary tracts. We found that the renal RI in healthy children is 
age-dependent. In the fasting state, $54 \%$ of non-obstructed renal units in children have an RI of 0.70 or higher. Intravenous infusion of normal saline and administration of furosemide can independently cause a significant decrease of the RI in non-obstructed renal units in children.

In the second clinical study (chapter 7), we investigated the effect of hyperhydration with normal saline and furosemide on renal RI in children with obstructive uropathy. We found that with an RI of 0.70 as the critical value for predicting renal obstruction, 82 versus $100 \%$ sensitivity $(\mathrm{P}<0.006), 63$ versus $94 \%$ specificity $(\mathrm{P}<0.04)$ and 76 versus $98 \%$ overall accuracy $(p<0.0005)$ were obtained for Doppler studies at baseline and after induced diuresis, respectively. From that study we concluded that Doppler ultrasonography after hyperhydration with normal saline and furosemide is an accurate method for diagnosing renal obstruction in children. It is more sensitive, specific and accurate than baseline Doppler studies.

In the third clinical study (chapter 8), we investigated the effect of hyperhydration with normal saline and furosemide on the renal RI in children with equivocal obstructive uropathy (children with $T_{1 / 2}$ values of 10-20 min). We found that in such children the determination of RI before and after infusion of normal saline and furosemide could be helpful in distinguishing obstructed from non-obstructed kidneys.

We conclude that renal RI is a useful method to identify patients with obstructive uropathy. However, at present, RI does not suffice if precise anatomical information is required. Therefore, it cannot be used as a single modality for evaluating a patient with hydronephrosis. RI provides corroboration of the initial diuretic renography and is considered as a non-invasive technique for monitoring the dilated collecting system under observation. Additionally, this method provides a non-invasive modality for monitoring patients after re-constructive surgery of the upper urinary tract. 


\section{SAMENVATTING}

Een belemmering van de afvloed van urine naar de blaas is een veel voorkomende afwijking zowel bij pasgeborenen als volwassenen. Een dergelijke afvloedbelemmering veroorzaakt verwijding van het boven de obstructie gelegen deel van de urinewegen. Meestal zal de nier verwijden (men spreekt dan van hydronefrose), soms ook de urinelijder. Door de obstructie zal de druk in het systeem oplopen met vermindering van nierfunctie als gevolg. De functie van de nier kan met behulp van isotopenonderzoek goed worden bepaald, maar de mate van obstructie is moeilijker vast te stellen. Bij lichte vormen van obstructie, waarbij de functie van de nier normaal blijft kan in het algemeen een afwachtende houding worden aangenomen. Is de obstructie ernstiger dan zal een operatieve behandeling moeten plaatsvinden om achteruitgang van de nierfunctie te voorkomen. Met name wanneer de functie van de nier nog normaal is, terwijl er wel een verwijding van het afvoersysteem wordt gevonden is het met de gangbare methoden niet altijd mogelijk vast te stellen of er inderdaad sprake is van een dusdanige mate van obstructie dat operatieve behandeling noodzakelijk is. In dit proefschrift wordt een nieuwe onderzoeksmethode (Doppler echografie) beschreven en vergeleken met de gangbare onderzoeken (echo en lasix renografie). De belangrijkste vraagstelling was te onderzoeken of deze nieuwe methode op een betrouwbaarder wijze de mate van obstructie zou kunnen vaststellen. Eerst is de methode getest in een proefdiermodel, in een latere fase is onderzoek bij patiënten verricht.

De in dit proefschrift beschreven ontwikkeling van een nieuw experimenteel diermodel met partiële obstructie van de ureter bleek eenvoudig, betrouwbaar en omkeerbaar te zijn (hoofdstuk 2). Het model is gebruikt om de gevolgen van unilaterale obstructie van verschillende ernst in drie experimenten te bestuderen.

In het eerste experimentele onderzoek (hoofdstuk 3 ) werden de effecten onderzocht van intraveneuze vocht toediening, met en zonder diureticum op de renale "resistive index" (RI) van geobstrueerde en niet geobstrueerde nieren. In unilaterale partiële obstructie, nam de RI na intraveneuze vochttoediening met furosemide toe bij obstructie en nam deze af in de niet-geobstrueerde nier. Een dergelijk divergerende reactie kan nuttig zijn voor de ontwikkeling van een farmacologisch onderzoek om geobstrueerde nieren beter te kunnen diagnosticeren.

In het tweede experiment (hoofdstuk 4) werden de veranderingen in nier RI en nierfunctie over een langere periode bestudeerd, gerelateerd aan verschillende graden van partiële obstructie en werd de correlatie bepaald tussen echografische en renografische bevindingen. Unilaterale partiële obstructie van de ureter veroorzaakte een toename van de Rl en de T1/2 en een afname van de effectieve renale plasma flow (ERPF) van de angedane nier, in combinatie met een afname van 
de RI en een toename van de ERPF van de contralaterale nier. Hoe ernstiger de mate van obstructie was, hoe groter de toename van RI en $T 1 / 2$ en de afname van ERPF bleek te zijn. Na stabilisatie van de obstructie waren RI en T1/2 positief gecorreleerd.

In het derde experiment (hoofdstuk 5), werden de veranderingen in RI en nierfunctie voor en na opheffen van de verschillende graden van unilaterale partiële obstructie bestudeerd.

Uit dit onderzoek kunnen de volgende conclusies worden getrokken:

1. unilaterale partiële ureter obstructie veroorzaakt een toename van de $\mathrm{Rl}$ en $\mathrm{T} 1 / 2$ en een afname van ERPF in de aangedane nier,

2. na opheffen van de obstructie neemt de nierfunctie weer toe met terzelfder tijd afname van de RI

3. functionele parameters (T1/2, RI of ERPF) van de geobstrueerde nier zijn geen graadmeters voor het herstel van ERPF na opheffen van de obstructie

4. een snel herstel van de aanvankelijk verhoogde $\mathrm{Rl}$ is een vroege indicator voor het herstel van nierfunctie na opheffen van een ureterobstructie

In aansluiting op de 3 experimentele onderzoeken werden een drietal klinische studies bij kinderen uitgevoerd. In het eerste onderzoek (hoofdstuk 6) werd het effect onderzocht van vasten, intraveneuze toediening van fysiologisch zout en de toediening van furosemide aan kinderen met normale hogere urinewegen op de Resistive Index. De RI bij gezonde kinderen bleek leeftijdsafhankelijk te zijn. Gedurende vasten bleek de RI bij 54\% van de niet-geobstrueerde kinderen hoger dan 0.70 te zijn. Intraveneuze toediening van fysiologisch zout en toediening van furosemide kunnen onafhankelijk van elkaar een significante afname van de RI in niet-geobstrueerde nieren veroorzaken.

In de tweede klinische studie (hoofdstuk 7) werd het effect bestudeerd van hyperhydratie met fysiologisch zout en furosemide op de RI in kinderen met een obstructieve uropathie. Uitgaande van een RI van 0.70 als de kritische grens voor voorspellende waarde van obstructie, werd een sensitiviteit van 82 vs 100 procent $(\mathrm{P}<0.006)$, een specificiteit van 63 vs 94 procent $(\mathrm{P}<0.04)$ en overall accuracy van 76 vs $98 \%$ $(\mathrm{P}<0.0005)$ vastgesteld voor Doppler studies tijdens basale waarden en geönduceerde diurese. Uit dit onderzoek blijkt dat Doppler onderzoek na hyperhydratie met fysiologisch zout en furosemide een nauwkeurige methode is om renale obstructie bij kinderen vast te stellen. Het is sensitiever, specifieker en nauwkeuriger dan basale Doppler onderzoeken.

In het derde klinische onderzoek (hoofdstuk 8) werd het effect van hyperhydratie met normaal fysiologisch zout en furosemide op de nier RI bij kinderen met een zogenaamde 'equivocal' obstructieve 
uropathie onderzocht. Dit is de groep kinderen waarbij op grond van een diurese renogram geen uitspraak kan worden gedaan of er nu wel of niet sprake is van een echte obstructie; er is een vertraagde washout met een $\mathrm{T} 1 / 2$ tussen de 10 en 20 minuten. Bij deze kinderen bleek de bepaling van Doppler RI voor en na toediening van fysiologisch zout en furosemide een goede methode te zijn om te differentiëren tussen geobstrueerde en niet-geobstrueerde systemen.

Concluderend kan worden gesteld dat het bepalen van de resistentieindex van de nier een bruikbare methode is om patiënten met een obstructieve uropathie te identificeren. Op dit moment is de nier RI echter niet in staat om nauwkeurige anatomische informatie te verschaffen. De resistentie-index kan daarom nog niet als op zichzelf staand onderzoek worden gebruikt om een patiënt met obstructieve uropathie te onderzoeken. Het is een goede aanvulling op het diurese renogram en kan heel goed worden gebruikt om als niet-invasief onderzoek een verwijd afvoersysteem te controleren wanneer wordt gekozen voor een conservatief beleid. Ook na een operatieve correctie van een obstructie van het afvoersysteem kan deze methode worden toegepast tijdens de periode van follow-up. 



\section{CURRICULUM VITAE}

$\begin{array}{ll}\text { NAME } & \text { : Ahmed Abdurrahman Ahmed Shokeir } \\ \text { DATE OF BIRTH } & \text { : July 5, 1956 El-Badrasheen, Geza } \\ \text { NATIONALITY } & : \text { Egyptian } \\ \text { SOCIAL STATUS } & : \text { Married }\end{array}$

CERTIFICATES:

- M.B., B.Ch. $1980 \quad$ Mansoura University, Egypt

- M.Sc. (Master) (Urology) 1986 Urology \& Neprology Center, Mansoura University, Egypt

- M.D. ( Doctorate) (Urology) 1990 Urology \& Neprology Center, Mansoura University, Egypt

\section{EXPERIENCE:}

1- Rotating Intership, Mansoura University Hospitals (12 months).

2- Resident in the department of Urology, Urology and Nephrology Center, Mansoura (48 months).

3- Assistant Lecturer in the department of Urology, Urology and Nephrology Center, Mansoura (48 months).

4- Lecturer in Urology, Urology and Nephrology Center, Mansoura between 1990-1995.

5- Visiting doctor at the University of Tennessee, Memphis, USA, 1991.

6- Associate Professor of Urology, Urology and Nephrology Center, Mansoura University Since 1996.

\section{PRESENT POSITION:}

Associate Professor of Urology, Urology and Nephrology Center, Mansoura University, Egypt. 
Shokeir AA, Gaballah MA, Ashamallah AA, Ghoneim MA 1991 Optimization of replacement of the ureter by ileum. Journal of Urology $146: 306-310$.

Shokeir AA, Bakr MA, El-Diasty TA, Sobh MA, Moustafa FE, El-Agroudi AE, Ghoneim MA 1992 Urological complications following live donor kidney transplantation: effect of urinary schistosomiasis. British Journal of Urology 70:247-25l.

Shokeir AA, Sobl MA, Bakr MA, Ghoneim MA 1992 Reimplantation ureterovesicale dans $\mathrm{La}$ transplantation renale d'un donneur vivant apparente: Extravesicale ou transvesicale? Complications urologiques et evaluations des results a long term. Progress en urologie 2: 241-248.

Sobh M, Yousif AE, Shokeir AA, Shaaban A, Kenawy M, El-Sherif A, Ghoneim M 1992 Impact of donor age on living related donor kidney transplantation. Transplant International 5 (Suppl 1): S727-S729.

Sobh MA, El-Agroudy AE, Moustafa FE, Shokeir AA, El-Shazly A, Ghoneim MA 1992 Impact of schistosomiasis on patient and graft outcome after kidney transplantation. Nephrology Dialysis and Transplantation. 7: 858-864.

Sobh MA, El-Sharkawy SE, Shokeir AA, Moustala FE, El-Sherif AK, Ghoneim MA 1992 Effects of schistosomiasis on living kidney donors. Scandinavian Joumal of Urology and Nephrology $26: 409-412$.

Ghaly AM, Shokeir AA, Abdallah HA, Ghoneim MA 1992 Orthotopic iteal bladder substitution in children with bladder exstrophy. New Egyptian Journal of Medicine Vol. 7 (No. 6): $1138-1142$.

Ghaly AM, Shokeir AA, Malıran MR, Sheir ZK, Ghoneim MA 1992 The modified rectal bladder (the augmented and valved rectum) for urine diversion in children. New Egyptian Journal of Medicine Vol. 7 (No. 6): 1134-1137.

Ghaly AM, Shamaa MA, Shokeir AA, Ghonein MA 1992 Augmentation ileocytoplasty for the management of incontinent epispadias. The New Egyptian Journal of Medicine Vol. 7 (No. 6) 1188-1190.

Shokeir AA, Shamaa MA, Bakr MA, Eraky I, Ghoneim MA 1993 lleal ureter in renal transplant recipients. Transplantation proceedings 25: 2339-2340.

Shokeir AA, El-Diasty TA, Shaaban, AA, El-Kenawey M, Eraky I, Ghoneim MA 1993 Evaluation of potential kidney donors using digital subtraction angiography. Transplantation proceedings 25: 2272-2273.

Shokeir AA, Gad HM, Shaaban AA, El-Kenawey MR, El-Sherif A, Bakr MA, Shamaa MA. Ghoneim MA 1993 Differential kidney scans in preoperative evaluation of potential kidney donors. Transplantation Proceedings 25: 2327-2329.

Shokeir AA, El-Diasty TA, Shanaa MA, Eraky I, Ghoneim MA 1993 Ureteric complications of renal transplantation: role of percutaneous teclniques. Transplantation proceedings 25: $2303-2304$. 
Bakr MA, Foda MA, Shokeir AA, Mostafa A, Sobl MA, Ghoneim MA 1993 Haemolytic anaemia after ABO unmatched live donor kidney transplantation. Transplantation Proceedings 25: 2297-2298.

Shonem MA, Sobh MA, Shokeir AA, Bakr MA, El-Sherif A, Foda MA 1993 Prospective randomized study of triple versus conventional immunosuppression in livedonor kidney transplantation. Transplantation Proceedings 25: 2243-2245.

Ghoneim, MA, Sobh MA, Shokeir AA, Bakr MA, El-Sherif A, Foda MA 1993 Cyclosporin in primary live donor kidncy transplantation: Is it wotthwhile? Nephrology Dialysis and Transplantation 8: 551-556.

Ghoneim, MA, Sobl MA, Shokeir AA, Bakr MA, El-Sherif A, Foda MA 1993 Prospcetive randomized study of azathioprine versus cyclosporin in live-donor kidney transplantation. Amcrican Journal of Nephrology 13: 437-441.

El-Diasty TA, Shokeir AA, El-Gharib MS, Sherif LS, Shamaa MA 1993 Bladder stone: A complication of intravesical migration pf lippes loop. Scandinavian Joumal of Urology and Neplarology 27: 279-280.

Shokeir AA, Eraky 1, Ghaly AM, Atallah K, Goma M 1993 Use of Swiss Lithoclast for transurethral cystolitholapaxy in children (Abstract). Journal of Endourology 7 Supplement 1 : S187.

Shokeir AA, EL-Diasty 'IA, Ghoneim MA 1993 Percutaneous treatment of lymplyocele in renal transplant recipients. Joumal of Endourology 7: 481-485.

Shokeir AA, El-Diasty TA, Ghoneim MA 1993 Endourologic management of ureteric complications following live-donor kidney transplantation. Journal of Endourology 7 : $487-491$

Shokeir AA, Mahran MR, Shamaa MA 1993 Interposition of ileum in the ureter. Scandinavian Joumal of Urology and Nephrology 27: $421-424$.

Shokeir AA, Shamaa MA, El-Diasty TA, Bakr MA, Ghoneim MA 1993 Salvage ol diffricult transplant urinary fistula by ilcal substitution of the ureter. Scandinavian loumal of Urology and Neplurology 27: $537-540$.

Sobh MA, Refaie A, Shokeir AA, Ghoneim MA 1994 Study of live donor kidney transplantation outcome in recipients with renal amyloidosis. Nephrology Dialysis and Transplantation 9: 704-708.

Shokeir AA, El-Kappany H, Ghoneim MA 1994 Accidental division of the tranplantated ureter during laparoscopic drainage of lymphocele. Joumal of Urology 151: 1623-1625.

Shokeir AA 1994 Transurethral cystolitholapaxy in children. Joumal of Endourology 8: $157-160$.

Shokeir AA, EI-Diasty TA, Shamaa MA, Ghoneim MA 1994 The Nutracker syndrome: New methods of diagnosis and treatment. British Journal of Urology $74:$ 139-143.

Shokeir AA, Eraky I, Hassan N, Wafa EW, Mohsen T, Ghoneim MA 1994 Tetracycline sclerotherapy for testicular hydroceles in renal transplant recipients. Urology 44: 96-99. 
Shokeir AA, El-diasty TA, Hassan NA, Wafa EW, Ghoneim MA 1994 Management of ureteric complications after renal transplantation. Egyptian Journal of Urology 1: 66-74.

Shokeir AA, El-Diasty TA, Ghoneim MA 1994 Percutaneous treatment of lymphocele in renal transplant recipients. Urology Digest, September 3: 13-14.

Shokeir AA, EL-Diasty TA, Ghoneim MA 1994 Endourologic management of uretcric complications after live-donor kidney transplantation. Urology Digest, September 3: 2425.

Shokeir AA, El-Diasty TA, Shaban A, et al. 1994 Intravenous digital substraction angiograplyy in potential live-kidney donors: A study of 1000 cases. Abdominal Imaging 19: $461-465$.

Shokeir AA, Moustafa FE, Abol-Enein H, Donia AF, Ghoneim MA 1994 Renal amyloidosis without urinary abnormality in a potential live-kidney donor. Nephrology Dialysis Transplantation 9: 1339-1340.

Slokeir AA, Shamaa MA, Abol-Enein 1I, EJ-Mekresh M, Ghoneim MA 1994 Postrenal transplantation urethral Kock pouch. Scandinavian Journal of Urology \& Nephrology 28: 315-318.

Shokeir AA.1995 Bladder cancer following ileal ureter: a case report. Scandinavian Journal of Urology \& Nephrology 29: 113-115.

Shokeir AA, Sobh MA, Gaber AO, Ghoneim MA 1994 Radioisotopic evaluation of renal function in cyclosporin treated pediatric and adult renal transplant recipients and their living donors: A study of 152 donor-recipient pairs. Transplantation 58: 1171-1175.

Shokeir AA, Ashamallah A, Abol-Enein H, Shokeir MA, Taqui AH 1995 lncomplete bladder duplication. British Jounal of Urology 75: 106-107.

Shokeil AA, Abol-Encin H, Shokeir MA, Taqui AH 1995 A ureteric stone in a child causing retention of urine. British Joumal of Urokogy 75: 102-103.

El-Diasty TA, Shokeir AA, Tawfeck HA, Mohamed NA, Nabeeh A, Ghoneim MA 1995 Ethanol sclerollierapy for treatment of symptonatic simple renal cysts. Journal of Endourology 9: 273-276.

Slwokeir AA, Shamaa MA, Eraky I, Abdel Gawad M, Ismail T 1995 Spontaneous passage of huge urcteric stone in a bilharzial patient. Scandinavian Journal of Urology \& Nephrology 29: $341-343$.

Shokeir AA, Dawaba M, Hafiz AT. Shokeir MA 1995 Giant bladder stone in a child. British Joumal of Urology 76: 659-670.

Shokeir AA, Ghoneim MA 1995 Further experience with the modilied ileal ureter. Journal of Urology 154: 45-48,

El-flammady S, Shokeir AA 1995 A novel teclnique of urcteroneocystostomy (extravesical seromuscular tunacl): An experinental study in dogs. I. Preliminary results. Urology 45: 339-343. 
Shokeir AA, El-Hammady S 1996 A novel teclnique of ureteroneocystostomy (extravesical seromuscular tunnel): Il. Optimization of surgical technique. Urology 48: 917-922.

Slwokeir $\Lambda$ A 1995 Partial ureteric obstruction: a new variable and reversible canine experimental mode. Urology 45: 952-957.

Shokcir AA, Dawaba, M, Cl-Azab M, Abdel-Gawad M, Shokeir, MA 1995 Prostatic abscess in a child. Scandinavian Jounal of Urology and Nephrology 29: 525-526.

Shokcir AA, El-Baz M, Abdel-Gawad M, Ali-Eldin B, Bazeed M 1995 Schistosomiasis: an tnusual cause of haematuria after ileal neobladder. Journal of Urology 154: 1129.

Shokeir AA, Shamaa M, El-Mekresh MM, El-Baz M, Ghoneim MA 1995 Late malignancy in bowel segments exposed to urine without fecal stream. Urology 46: 657661 .

Shokeir AA, Ill-Diasty TA, Gad IJM, Sobh MA, El-Sherif $\Lambda$ K, Gaber AO, Ghonem MA 1995 Radioisotopic evaluation of renal function in cyclosporin treated pediattic and adult renal transplant recipients and their living donors: A study of 152 donor-recipient pairs. 1. (frol, 153:2080-2081, (Abstract). liditorial Comment by Androw Novick.

Shokeir AA, El-Diasty TA, Ghonein MA 1995 Percutaneous treatment of lymphocele in renal transplant recipients. J. Urol. 153:2081-2082, (Abstract). Editorial Comment by Androw Novick.

E1-Diasty TA, Shokeir AA, Tawfeek HA, Mahmoud NA, Nabeeh A, Gloneim MA 1995 T:thanol sclerotherapy for symptomatic simple renal cysts. Urology Digest 2: 17 19.

El-Azab M, Mohsen T, El-Diasty T, Shokeir AA 1996 Doppler ultrasonograply in evaluation of potential live-kidney donors: A prospective study. 3. Urol 156: 878-880.

Shokeir A . Provoost AP. El-Azab M. Dawaba M, Nijman RJM 1996 Relal Doppler ultrasound in children with nomal upper urnary tracts: Effects of fasting, hydration with nomal saline and furosemide administration. Urology 47: 740-744.

Sliokeir AA, Nijman R.IM, El-Azab M, Provoost AP 1996 Partial ureteral obstruction. A study of Doppler ultrasonography and diuretic renography in different grades and durations of obstruction. British Journal of Urology 78: 829.835 .

Shokeir AA, Provoost AP, El-Azab M, Dawaba M, Nijman RJM 1996 Renal Doppler uhrasound in children with obstructive uropathy: Effect of intravenous normal saline 1luid load and furosemide. J. Urol 156: 1455-1458.

Shokeir M, Shokeir AA. Dawaba M. Emara S, Gabaliah M 1996 Pediatric urolithiasis: Clinical experience. The Egyptian loumal of Pacdiatrics 13 (No. I-2): 235-245 (Supplement).

Shokeir AA. Nijman R.JM, El-Azab M. Provoost MP 1997 partial ureteral obstruction. Role ol renal resistive index in stages of obstruction and release. Urology 49: 528-5.35.

Shukeir $\Lambda$ A, Nijiman R.JM. Jil- $\mathrm{zab}$ M, Provonst AP 1997 Partial ureteral obstruction: Effect of intravenous nomal saline and furosemide upon the renal resistive index. J. Urol 157: 1074-1077. 
Slokeir AA, El-Azab M, Mohsen T, El-Diasty TA 1997 Emphysematous pyelonephritis: A 15-year experience with 20 cases. Utology 49: 343-346.

Shokeir AA 1997 Interposition of iteum in the ureter: A clinical study with long-term follow -up British Journal of Urology 79: 324-327.

Shokeir AA, Provoost AP, El-Azab M, Dawaba M, Shokeir MA, Nijman R.iM 1997 Renat Doppler uttrasound in children with equivocal obstructive uropathy: Effect of intravenous normal saline fluid load and furosemide. British Journal of Urology 80: 313-318.

Slokeir AA, Provoost AP, Nijman RJM 1997 Resistive index in obstructive uropathy " Review Article". British Journal of Urology 80: 195-200.

Shokeir AA, Al Sisi H, Farag YM, Abd El-Maaboud M, Saeed M, Mutabagani H 1997 Transuretlural prostatectomy: A prospective randomized study of conventional TURP versus electrovaporization in benign prostatic hyperplasia. British Journal of Urology 80 : $570-574$.

Shokeir AA. 1997 Bladder Cancer following: Leal ureter replacement. Review Series UROLOGY Issue 2: 14-15.

Shokeir AA, El-Baz M, El-Diasty TA, El-Mekresh MM, Abdel-Gawad M 1997 Multiplc but different urothelial tumours in one side of a urinary tract. Scandinavian Journal of Urology \& Nephrology 31: 199-201.

Shokeir AA Mutabagani H. 1998 Rigid Ureteroscopy in pregnant women. British Journal of Urology 81: 678-681.

Abdulmaaboud M, Shokeir AA, Farage Y, Abd El-Rahman A, El-Rakhawy MM, Mutabagani H 1998 Treatment of Varicocele: a comparative study of conventional open surgery, percutaneous retrograde sclerotherapy and laparoscopy. Urology 52:294-300.

Slsokeir AA, El-Hammady S 1998 Extravesical Seromuscular tunnel: a novel techuique of ureteroneocystostomy (Point of technique). British loumal of Urology (In press).

Shokeir AA, Provoost AP, Nijman RJM 1998 Prediction of recoverability of renal function after relief of chronic partial upper urinary obstruction (Review Article). British Journal of Urology (in press).

Shokeir AA, Abdelmaaboud M 1998 Resistive index in renal colic: a prospective study. British Journal of Urology (ln press).

Shokeir AA, Alserafi MA, Mutabagani H 1998 Intracavenous versus Intraurethral Alprostadil: a prospective randonized study. British Journal of Urology (In Press) .

Shokeir AA 1998 Urology of the Pharaohs (Names In Genitourinary Surgery). (Subnitted).

Shokeir AA 1998 Renal transplantation: Impact of schistosomiais (Review Article). (In preparation). 


\section{A C K N OWLED GEMEN T S}

Thanks to Prof. M. A. Ghoneim who gave me the opportunity to start this work.

Thanks to Prof. F. H. Schroder for his invaluable help and support.

Thanks to Dr. Rien J. M. Nijman who helped me so much during the whole course of this thesis.

Thanks to Dr. A.P. Provoost for his great support and sincere assistance. 




